\title{
Summary of BISON Development and Validation Activities - NEAMS FY16 Report
}

\author{
R. L. Williamson \\ G. Pastore \\ K. A. Gamble \\ B. W. Spencer \\ A. Casagranda \\ C. P. Folsom \\ W. Liu \\ S. Veeraraghavan \\ S. R. Novascone \\ R. J. Gardner \\ J. D. Hales
}




\section{NOTICE}

This information was prepared as an account of work sponsored by an agency of the U.S. Government. Neither the U.S. Government nor any agency thereof, nor any of their employees, makes any warranty, express or implied, or assumes any legal liability or responsibility for any third party's use, or the results of such use, of any information, apparatus, product, or process disclosed herein, or represents that its use by such third party would not infringe privately owned rights. The views expressed herein are not necessarily those of the U.S. Nuclear Regulatory Commission. 
Summary of BISON Development and Validation Activities NEAMS FY16 Report

\author{
R. L. Williamson \\ G. Pastore \\ K. A. Gamble \\ B. W. Spencer \\ A. Casagranda \\ C. P. Folsom \\ W. Liu*
}

S. Veeraraghavan

S. R. Novascone

R. J. Gardner

J. D. Hales

*ANATECH, Corp.

September 2016

Idaho National Laboratory

Fuel Modeling and Simulation Department

Idaho Falls, Idaho 83415

\author{
Prepared for the \\ U.S. Department of Energy \\ Office of Nuclear Energy \\ Under U.S. Department of Energy-Idaho Operations Office \\ Contract DE-AC07-99ID13727
}




\section{Contents}

\begin{tabular}{lll}
\hline & Introduction & 6
\end{tabular}

$\begin{array}{|lll|}2 & \text { Milestone Summary } & \mathbf{7}\end{array}$

$2.1 \quad$ Milestone Level and Completion Schedule . . . . . . . . . . . . . . . . . . 7

2.2 Release BISON Update for LWR Fuel Performance in Quasi-Steady, Off-Normal

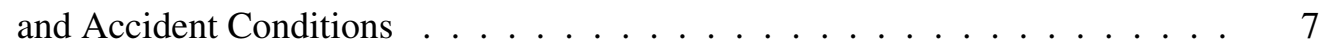

2.3 Issue Update to BISON Validation Report . . . . . . . . . . . . . . . . . . 8

2.4 Improve BISON software quality practices and participate in independent SQA

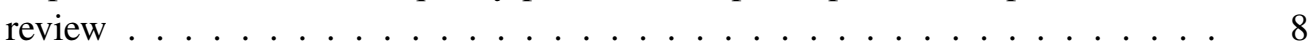

2.5 Develop material models for accident behavior especially rate and temperature

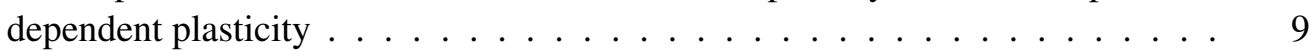

2.5 .1 Cladding Failure (Burst) Criterion . . . . . . . . . . . . . . . 11

2.5 .2 Time Increment Control . . . . . . . . . . . . . . . . . . . 12

2.6 Simulate FUMAC priority cases and participate in mid-project meeting . . . . 13

2.7 Improve oxide mechanics in 3D especially the robustness and efficiency of smeared

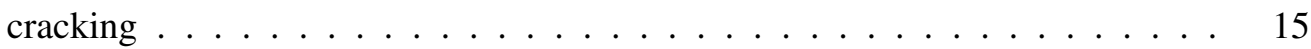

2.7 .1 Introduction . . . . . . . . . . . . . . . . . . . . . . 15

2.7 .2 Code Improvements . . . . . . . . . . . . . . . . . . . . . 15

$2.7 .3 \quad$ Results $\ldots \ldots \ldots \ldots \ldots \ldots \ldots$

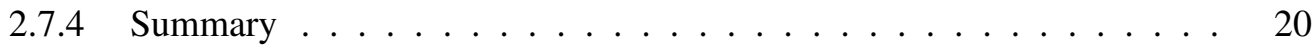

$\begin{array}{lll}3 & \text { Additional Major Accomplishments } & 21\end{array}$

3.1 BISON Validation Summary Article . . . . . . . . . . . . . . . . . . . . . . . 21

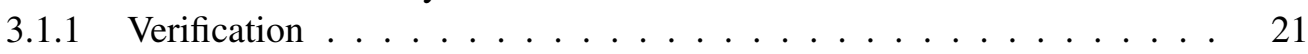

3.1 .2 Experimental data used for validation . . . . . . . . . . . . . 21

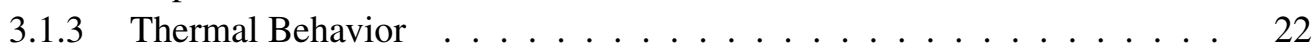

3.1 .4 Fission Gas Release $\ldots \ldots \ldots$

3.1 .5 Rod Diameter . . . . . . . . . . . . . . . . . . . . . . . . . . . 24

3.1 .6 Summary . . . . . . . . . . . . . . . . . . . 26

3.2 Missing Pellet Surface Article $\ldots \ldots \ldots \ldots$. . . . . . . . . . . . 28

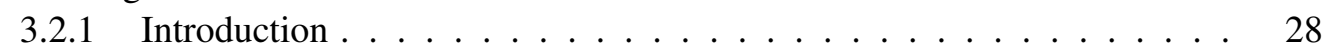

3.2 .2 Models $\ldots \ldots \ldots \ldots \ldots \ldots$

3.2 .3 Results . . . . . . . . . . . . . . . . . . . 30

3.3 Halden 3D MPS Experiment . . . . . . . . . . . . . . . . . . . . . 30

3.3 .1 Introduction . . . . . . . . . . . . . . . . . . 30

3.3 .2 Fuel Rod Configuration $\ldots \ldots \ldots \ldots$. . . . . . . . . . . . 32

3.3 .3 Models $\ldots \ldots \ldots \ldots \ldots \ldots$. . . . . . . . . . . . . . . . . . . 32 
3.3 .4 MPS Defect Depth Effect $\ldots \ldots$. . . . . . . . . . . . . . . 33

3.3 .5 Contact Model Comparison . . . . . . . . . . . . . . . . . . . . 34

3.3 .6 Conclusions . . . . . . . . . . . . . . . . . . . 35

3.4 Pellet Clad Mechanical Interaction Benchmark _ . . . . . . . . . . . . . . 37

$3.4 .1 \quad$ Background and Introduction . . . . . . . . . . . . . . 37

3.4 .2 Benchmark Cases and Model Status . . . . . . . . . . . . . . . . . . . . . . . . . 37

3.4 .3 Early Results and Comparisons _. . . . . . . . . . . . . . . 38

3.4 .4 Summary . . . . . . . . . . . . . . . . . . . . . 38

3.5 OECD Reactivity Insertion Accident Benchmark . . . . . . . . . . . . . . 40

$3.5 .1 \quad$ Background and Introduction $\ldots \ldots \ldots \ldots$. . . . . . . . . 40

3.5 .2 Benchmark Specifications $\ldots \ldots \ldots . \ldots . \ldots 41$

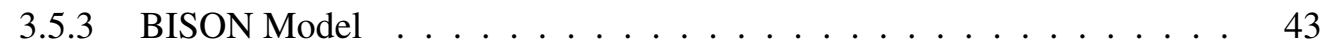

3.5 .4 Results $\ldots \ldots \ldots \ldots$. . . . . . . . . . . . . . . . . . . . . . 46

3.5 .5 Summary . . . . . . . . . . . . . . . . . . . . 48

$3.6 \quad$ Uncertainty Quantification and Sensitivity Analysis in Fuel Modeling . . . . . 49

3.7 Improved Fission Gas Behavior Model . . . . . . . . . . . . . . . . . 53

3.7.1 New Algorithm for Fission Gas Diffusion with Improved Accuracy . . 54

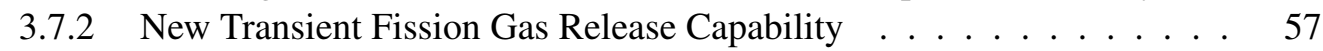

4 Future Work 62

5 Acknowledgments

\begin{tabular}{ll}
\hline Bibliography & 64
\end{tabular} 


\section{Introduction}

The objective of the NEAMS ToolKit is to develop a "pellet-to-plant" simulation capability useful for predicting performance and safety for a broad range of nuclear reactor power systems. The NEAMS ToolKit has been organized into a Fuels Product Line (FPL) and a Reactor Product Line (RPL) and is modular in design. Within the FPL, a multiscale approach has been adopted in which simulations of fuel performance at the engineering scale are informed by material property and irradiation performance models developed from mesoscale simulations of microstructural evolution. The focus in this report is on development and validation of the engineering-scale fuel performance analysis tool within the FPL, which is BISON [1].

This summary report contains an overview of work performed under the work package entitled "FY2016 NEAMS INL-Engineering Scale Fuel Performance (BISON)" A first chapter identifies the specific FY-16 milestones, providing a basic description of the associated work and references to related detailed documentation. Where applicable, a representative technical result is provided. A second chapter summarizes major additional accomplishments, which include: 1) publication of a journal article on solution verification and validation of BISON for LWR fuel, 2) publication of a journal article on 3D Missing Pellet Surface (MPS) analysis of BWR fuel, 3) use of BISON to design a unique 3D MPS validation experiment for future installation in the Halden research reactor, 4) participation in an OECD benchmark on Pellet Clad Mechanical Interaction (PCMI), 5) participation in an OECD benchmark on Reactivity Insertion Accident (RIA) analysis, 6) participation in an OECD activity on uncertainty quantification and sensitivity analysis in nuclear fuel modeling and 7) major improvements to BISON's fission gas behavior models. A final chapter outlines FY-17 future work. 


\section{Milestone Summary}

\subsection{Milestone Level and Completion Schedule}

FY-2016 Milestones and the completion dates are listed in Table 2.1. Following sections contain a short description of each milestone and references to related detailed documentation. Where applicable, a representative technical result from the work is included.

Table 2.1: FY-2016 Milestones for NEAMS INL-Engineering Scale Fuel Performance Effort

\begin{tabular}{lcc}
\hline Milestone & Completion Date & MS Level \\
\hline $\begin{array}{l}\text { Release BISON update for LWR fuel performance in } \\
\text { quasi-steady, off-normal and accident conditions. }\end{array}$ & $9 / 30$ & M2 \\
\hline $\begin{array}{l}\text { Issue update to BISON validation and assessment report } \\
\begin{array}{l}\text { Improve BISON software quality practices and } \\
\text { participate in independent SQA review }\end{array}\end{array}$ & $9 / 30$ & M2 \\
\hline $\begin{array}{l}\text { Develop material models for accident behavior especially } \\
\text { rate and temperature dependent plasticity }\end{array}$ & $4 / 28$ & M3 \\
\hline $\begin{array}{l}\text { Simulate FUMAC priority cases and participate in } \\
\text { mid-project meeting }\end{array}$ & $6 / 30$ & M3 \\
\hline $\begin{array}{l}\text { Improve oxide mechanics in 3D especially the } \\
\text { robustness and efficiency of smeared cracking }\end{array}$ & $8 / 30$ & M3 \\
\hline
\end{tabular}

\subsection{Release BISON Update for LWR Fuel Performance in Quasi-Steady, Off-Normal and Accident Conditions}

The major accomplishment for this year was the release of an updated version of BISON (Version 1.3) with corresponding documentation including an updated user [2] and theory manual [3]. The BISON training materials were also significantly improved to include more basic thermomechanics example problems and more detailed information on fuels-specific models. The major new or improved capabilities in Version 1.3 include:

- Improved mechanical contact performance and robustness

- Improved hybrid approach for frictional contact

- Improvements in robustness of the existing anisotropic smeared fracture model 
- Addition of a new simplistic isotropic smeared fracture model

- Capability to map 2D-RZ field variables (temperature and displacement) for a full-length fuel rod to either a $2 \mathrm{D}$ generalized plane strain or 3D submodel

- Improvements to fission gas behavior models including a new algorithm for gas diffusion with improved accuracy and a new transient gas release capability

- Material models for ZIRLO cladding

- Update of the radial power profile model to include gadolinium fuel

- Instantaneous plasticity of Zircaloy as a function of temperature, irradiation and strain rate

- Improvements to fast metal and MOX material models (this development was funded principally by the Advanced Fuel Campaign)

\subsection{Issue Update to BISON Validation Report}

The BISON Light Water Reactor (LWR) validation base was enlarged by adding separate effects experiments and several integral rod cases. The report was also updated to include a section on validation to Loss of Coolant Accident (LOCA) behavior. All cases considered to date are documented in the updated BISON Validation report [4].

\subsection{Improve BISON software quality practices and participate in independent SQA review}

An independent NQA-1 review of MOOSE/BISON software quality practices was performed by Michael Lackner (LANL Software Quality Engineer) and Nancy Kyle (Partner and Principal Consultant, Theseus Professional Services), during the week of November 16-20, 2015. In preparation for the assessment, four documents were prepared as outlined below. These documents are available to code developers and users via links on the BISON Gitlab website.

1. Project Management Plan for MOOSE-Based Applications, INL Technical Report PLN4213, Rev 1, 11/16/2015 (Project planning information; High level overview touching on our entire project and software development)

2. Configuration Management Plan for MOOSE and MOOSE-Based Applications, INL Technical Report PLN-4004, Rev 4, 3/16/2016 (Roles and Responsibilities, Pull Request/Merge Request Workflow and Workflow Diagram)

3. Software Quality Assurance Plan for MOOSE and MOOSE-Based Applications, INL Technical Report PLN-4005, Rev 3, 3/16/2016 (Overall plan for developing software; Contains document references to other relevant plans and procedures; Contains information about Safety Software Determination and Quality Level Determination) 
4. Verification and Validation Plan for MOOSE and MOOSE-Based Applications, INL Technical Report PLN-4006, Rev 3, 3/16/2016 (Definitions, methods and procedures for software $\mathrm{V} \& \mathrm{~V}$ )

During the assessment, reviewers interviewed management, the BISON development team, and a small group of BISON users. Interviews included review and discussion of the above software quality documents and probing questions to determine actual application and adherence to these processes. A number of formal comments were provided concerning the documents, which were addressed and submitted to the reviewers in preparation for a second assessment the week of March 21, 2016.

At the November assessment, the reviewers deemed MOOSE/BISON NQA-1 compliant for R\&D software. The only substantial findings of the assessors were:

- The absence of a formal document and process that connects BISON software requirements to unit testing

- The need for a more complete Software Design Description

These findings resulted in the development of the draft report entitled, "BISON Software Requirements Specification and Software Design Description" which was submitted to the same NQA-1 reviewers during an assessment follow-up meeting the week of March 21, 2016. We await the results from that assessment.

\subsection{Develop material models for accident behavior especially rate and temperature dependent plasticity}

A Zircaloy plasticity model applicable to high temperatures and strain rates was recently implemented from [5]. Before yield, the stress-strain relationship follows Hooke's law, i.e.,

$$
\sigma=E \varepsilon
$$

where $\sigma$ is the stress, $\varepsilon$ is the total strain and $E$ is the Young's modulus.

After yield, the stress-strain relationship follows a power law as shown below

$$
\sigma=K \varepsilon^{n}\left(\frac{\dot{\varepsilon}}{10^{-3}}\right)^{m}
$$

where $K$ is the strength coefficient, $n$ is the strain hardening exponent, $m$ is the strain rate exponent and $\dot{\varepsilon}$ is the strain rate. Note that the total strain $(\varepsilon)$ is used in the above expression.

The yield stress $\left(\sigma_{y}\right)$ is then the non-zero intersection of the above two equations and is given by

$$
\sigma_{y}=\left[\frac{K}{E^{n}}\left(\frac{\dot{\varepsilon}}{10^{-3}}\right)\right]^{\left(\frac{1}{1-n}\right)}
$$

In this material model, the Young's modulus $E$ is a function of temperature of the cladding, fast neutron fluence, cold work factor and oxygen concentration and is calculated using the 
MATPRO material model CELMOD. It should be noted that the Young's modulus calculation is done using the MechZry model. So the MechZry model is a requirement for the ZryPlasticity model to produce accurate results. The strength coefficient $K$, strain hardening exponent $n$ and strain rate exponent $m$ are functions of the cladding temperature, fast neutron fluence, fast neutron flux and cold work factor and the expressions for these are given in [5]. To account for the effect of annealing, the MATPRO material model CANEAL is used to correct the cold work factor and fast neutron fluence.

To use this model with the return mapping algorithm, the stress after yield needs to be written in terms of the plastic strain $\left(\varepsilon_{p}\right)$ instead of the total strain $(\varepsilon)$. This can be achieved by substituting $\sigma / E$ for the elastic strain. Therefore, the stress-plastic strain relation after yield can be written as

$$
\varepsilon_{p}=\left[\frac{\sigma}{K}\left(\frac{10^{-3}}{\dot{\varepsilon}}\right)^{m}\right]^{\frac{1}{n}}-\frac{\sigma}{E}
$$

A comparison between the simulated uniaxial test and the experiment is presented in Figure 2.1. Here, a block of Ziracloy with fluence of $10.3 \times 10^{5} \mathrm{n} / \mathrm{m}^{2}$ at a temperature of $673 \mathrm{~K}$ is uniaxially pulled at a constant strain rate of $4.17 \times 10^{-5} \mathrm{~s}^{-1}$. The stress-strain curve for this scenario is shown in Figure 2.1. The degradation in the yield stress of the Ziracloy material as a function of temperature is shown in Figure 2.2 .

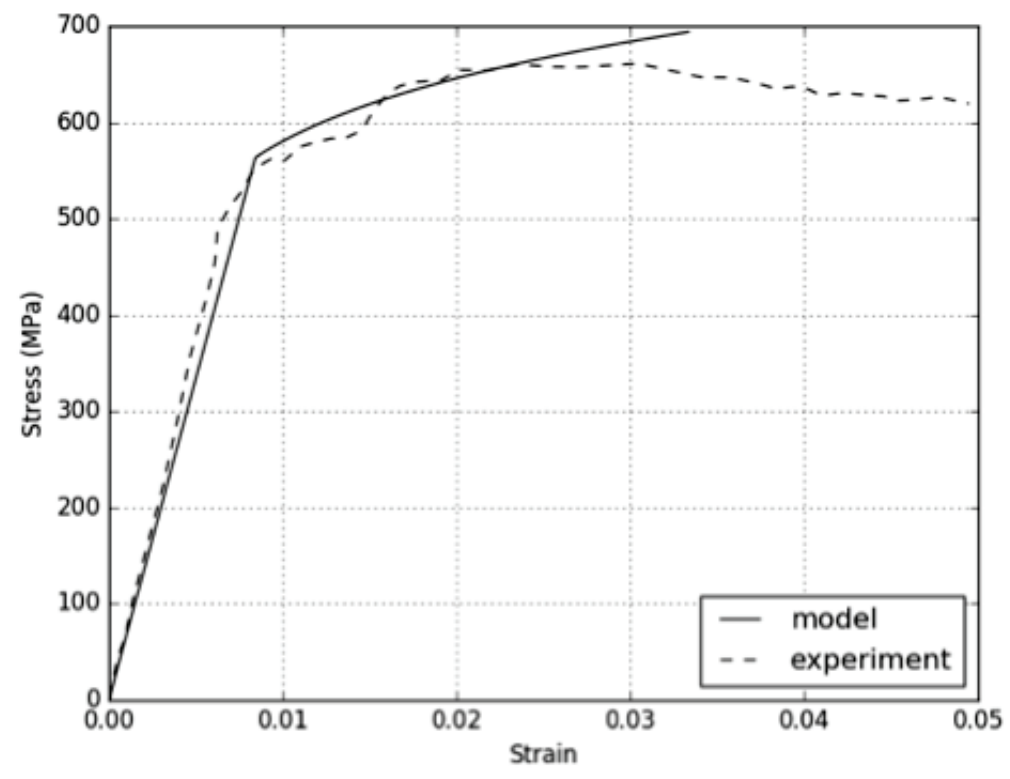

Figure 2.1: Comparison between experiments and simulation for uniaxial test conducted on Ziracloy. 


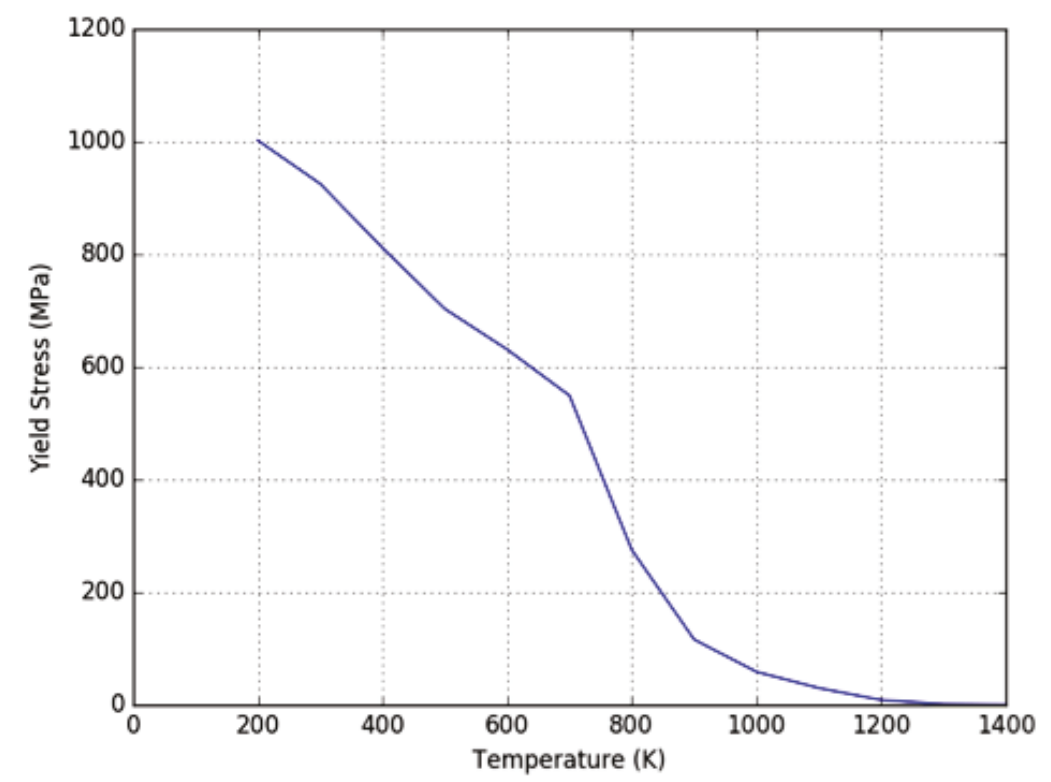

Figure 2.2: Yield stress of Ziracloy material as a function of temperature.

\subsubsection{Cladding Failure (Burst) Criterion}

For modeling burst failure of Zircaloy-4 cladding due to microcracking, BISON offers three options:

1. An overstress criterion, which assumes that the time of burst is reached when the local hoop stress equals a limiting burst stress [6]:

$$
\sigma_{\theta} \geq \sigma_{b}
$$

where $\sigma_{\theta}(\mathrm{MPa})$ is the hoop stress and $\sigma_{b}(\mathrm{MPa})$ is the burst stress.

2. A plastic instability criterion, which considers cladding burst at the attainment of a limiting value for the effective plastic strain rate:

$$
\dot{\varepsilon}_{p l, e f f} \geq \dot{\varepsilon}_{b}
$$

where $\dot{\varepsilon}_{p l, e f f}$ is the effective plastic (creep + plasticity) strain rate and $\dot{\varepsilon}_{b}$ is the limiting value. Following [7], we choose $\dot{\varepsilon_{b}}=100 \mathrm{~h}^{-1} \cong 2.78 \cdot 10^{-2} \mathrm{~s}^{-1}$.

3. A combination of the above criteria, which establishes that cladding burst occurs when either condition 2.5 or 2.6 is fulfilled.

The calculation of the burst stress follows the work of Erbacher et al. [6]. Based on experimental evidence, the burst stress is considered to depend on the temperature and oxygen concentration in the cladding, and is represented by [6]: 
Table 2.2: Material parameters used to calculate the burst stress of Zircaloy-4 [6].

\begin{tabular}{lll}
\hline Phase & $\mathrm{a}(\mathrm{MPa})$ & $\mathrm{b}\left(\mathrm{K}^{-1}\right)$ \\
\hline$\alpha$ & 830 & $1 \cdot 10^{-3}$ \\
$50 \% \alpha 50 \% \beta$ & 3000 & $3 \cdot 10^{-3}$ \\
$\beta$ & 2300 & $3 \cdot 10^{-3}$ \\
\hline
\end{tabular}

$$
\sigma_{b}=a \cdot \exp (-b T) \cdot \exp \left[-\left(\frac{\eta-\eta_{0}}{9.5 \cdot 10^{-4}}\right)^{2}\right]
$$

where a (MPa) and $\mathrm{b}\left(\mathrm{K}^{-1}\right)$ are constants determined experimentally, and $\eta(-)$ is the oxygen weight fraction in the cladding. An oxygen weight fraction at fabrication, $\eta_{0}=1.2 \cdot 10^{-3}$, is considered [6]. The current oxygen weight fraction is computed based on the oxygen mass gain from the BISON oxidation model as:

$$
\eta=\frac{2 r_{c l, o}}{\rho_{Z y} \cdot\left(r_{m e t, o}^{2}-r_{c l, i}^{2}\right)} \cdot g+\eta_{0}
$$

where $r_{c l, o}(\mathrm{~m})$ is the cladding outer radius, $\rho_{Z y}=6550 \mathrm{~kg} \cdot \mathrm{m}^{-3}$ the density of the cladding metal, $r_{c l, i}(\mathrm{~m})$ the cladding inner radius, $\mathrm{g}\left(\mathrm{kg} \cdot \mathrm{m}^{-2}\right)$ the oxygen mass, and $r_{m e t, o}=r_{c l, o}-S / R_{P B}$ with $\mathrm{S}(\mathrm{m})$ being the oxide layer thickness and $\mathrm{R}_{P B}=1.56$ the Pilling-Bedworth ratio for $\mathrm{Zr}$. The values for the parameters $\mathrm{a}$ and $\mathrm{b}$ are given in Table 2.2. In the mixed phase $(\alpha+\beta)$ region, linear interpolations of $\ln (a)$ and $b$ are made between the values for pure $\alpha$ and middle of $\alpha+\beta$ $(50 \% \alpha 50 \% \beta)$ phase, and between $50 \% \alpha 50 \% \beta$ and pure $\beta$ phase [6]. BISON includes a phase transformation model to compute the volume fractions.

Note that these models have been calibrated using burst data for power ramp rates much slower than those expected in a RIA and thus must be validated against RIA experiments before being used with confidence.

\subsubsection{Time Increment Control}

In addition to implementing the new stress-strain relationship as described above, in order to improve the numerical solution in presence of non-linear material behavior (plasticity, creep) during accident situations, a new automatic time step control was developed for BISON. In particular, a time step criterion physically based on the strain rate of the material was implemented. The criterion limits the time step length to guarantee that the increment of inelastic strain during the time step is kept under a pre-defined limiting value:

$$
\Delta t \leq \frac{\Delta \varepsilon_{c r, l i m}}{\dot{\varepsilon}_{c r}}
$$

where $\Delta t$ is the time step length, $\Delta \varepsilon_{c r, l i m}$ is the limiting value of creep strain increment, and $\dot{\varepsilon}_{c r}$ is the creep strain rate. As the creep strain rate is different for different locations in the cladding, 
the most restrictive condition (maximum strain rate across the domain) is considered. This is enforced through a dedicated postprocessor. The new criterion allows for automatic control of the numerical error due to time discretization in presence of non-linear material behavior, thereby guaranteeing a suitable accuracy of the numerical solution. This is important during situations involving high strain rates such as RIA and LOCA accidents. The criterion is combined with the current time step control capability in BISON. A flexible programming structure was set up and in perspective this time stepping method can be extended to incorporate further physically based criteria.

\subsection{Simulate FUMAC priority cases and participate in mid-project meeting}

The FUel Modeling Under Accident Conditions (FUMAC) is an IAEA sponsored Coordinated Research Project (CRP) designed to improve and validate nuclear fuel performance codes for accident analysis, with a focus on Loss-Of-Coolant Accidents (LOCA). It is a three-year program involving IAEA member states from throughout the world. The project provides access to high quality validation data, a chance to interface closely with international nuclear fuel experts, and an opportunity to compare BISON capabilities and predictions to several well- known fuel performance codes.

BISON developers Rich Williamson and Giovanni Pastore participated in the planning and organization of the project and attended the first coordination meeting held in 2014. The midproject coordination meeting was held in Vienna, Austria on May 30 through June 3, 2016. Rich Williamson and Giovanni Pastore participated in the meeting and provided an update on INL accomplishments during the first half (18 months) of the CRP. This work led to BISON developments for LOCA analysis and simulation of several FUMAC priority cases. More details on these simulations are given below. The BISON presentation was positively received and got interesting feedback that will help in identifying optimized development directions and enhancing future work.

FUMAC priority cases were simulated with BISON, including both separate effects and integral fuel rod tests. In particular, BISON analyses were performed of the following cases:

- MTA-EK separate effects tests PUZRY [8]

- EON segment 2 [9]

- Halden integral fuel rod test IFA-650.2 [10]

- Halden integral fuel rod test IFA-650.10 [11]

In addition, the REBEKA separate effects tests [12] of cladding ballooning and burst under LOCA conditions were analyzed with BISON for a more extensive code assessment. Some of the main BISON results for the simulation of the FUMAC priority cases are presented hereinafter.

The PUZRY test series belongs to the MTA-EK database on separate effects ballooning and burst tests for Zircaloy (specifically, Zy-4) claddings. All 31 tests from the PUZRY series have 
been analyzed with BISON. The results in terms of cladding internal pressure at burst and time to burst are compared to the experimental data in Figure 2.3. The accuracy of BISON analyses is reasonable and in line with state-of-the-art predictions with other fuel performance codes.

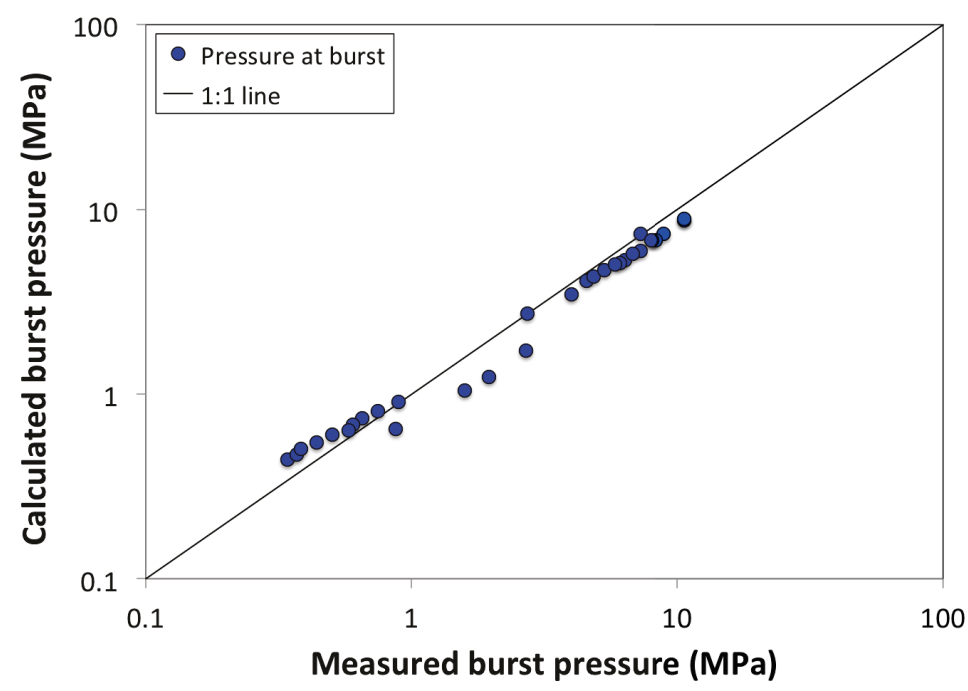

(a)

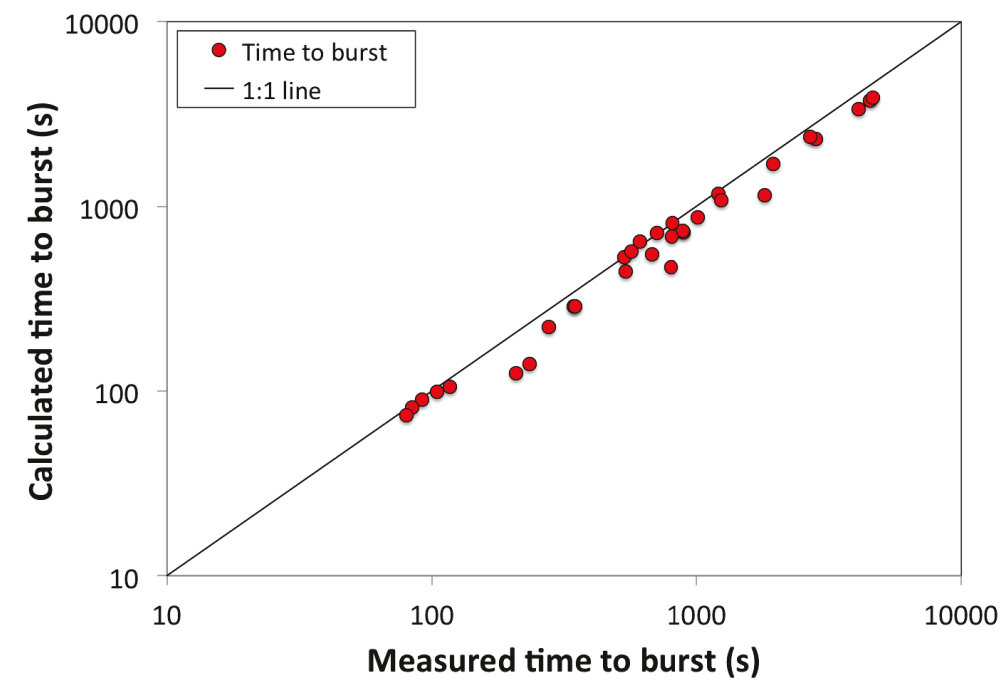

(b)

Figure 2.3: Comparison between BISON predictions and experimental data of cladding burst pressure (left) and time to burst (right) for the simulations of the MTA-EK separate effects tests PUZRY.

Two Halden LOCA cases were analyzed with BISON, namely, the second trial tests IFA-650.2 and the tenth test IFA-650.10. For the simulation of IFA-650.2, simplified thermal boundary conditions based on the available outer cladding thermocouple measurements were developed. The 
BISON simulation of the IFA- 650.10 test included the base irradiation based on the available information and power history. The thermal boundary conditions during the base irradiation were determined using BISONs internal coolant channel model. Instead, for simulating the LOCA transient test in the Halden reactor, the thermal boundary conditions calculated at IBRAE (Russian Federation) with the SOCRAT code and provided through FUMAC were used to inform the BISON calculation. In Figure 2.4 the results of inner pin pressure during the LOCA transient calculated by BISON for the IFA-650.2 and IFA-650.10 tests are compared to the on-line experimental measurements. Calculated and experimentally determined times to burst are also illustrated. Predictions are reasonably accurate for both cases. The predicted time to burst is within $7 \mathrm{~s}$ of the experimental one for IFA-650.2, and within $9 \mathrm{~s}$ of the experimental one for IFA-650.10.

\subsection{Improve oxide mechanics in 3D especially the robustness and efficiency of smeared cracking}

\subsubsection{Introduction}

Thermal stress as a result of radial temperature gradients in ceramic $\mathrm{UO}_{2}$ fuel can cause cracking under operating conditions, with cracks initiating at the peripheral region of fuel at relatively low temperatures. Fuel cracking has implications on fuel performance in both normal operation and transient conditions. Extensive fuel cracking enables the movement of fuel fragments, i.e., to relocate fuel fragments which can affect fuel temperature (and stored energy) significantly. Cracked fuel in contact with cladding will result in uneven stress distributions on the cladding, and high stress concentration is one of the important factors contributing to Pellet-Cladding Interaction (PCI) failure. Fuel cracking and relocation in current fuel performance codes are often modeled empirically. To improve the modeling and simulation of fuel behavior, a mechanistic material modeling approach is desired. A smeared-cracking model has been implemented in BISON to simulate fuel fracture where cracking is modeled as a mechanism that alters the fuel stiffness by changing the material property from isotropic to orthotropic. The smeared crack model does not track each individual discrete crack, instead, a strain softening curve is employed as part of the constitutive law to describe the post-cracking response. Constitutive equations are solved at each integration point in the finite element model.

Extensive testing has been performed on the use of the smeared cracking model in the past. It has been shown that the model can produce reasonable results with converged solutions on simple problems. However, in modeling more realistic fuel rod problems, the smeared cracking model was not always satisfactory, and could experience numerical convergence problems. The present work improves the numerical robustness of the of smeared cracking model.

\subsubsection{Code Improvements}

The smeared cracking numerical method and its implementation in BISON has been reviewed. A number of changes and tests have been made to improve the $\mathrm{UO}_{2}$ mechanical models:

- Addition of a ductile-brittle transition temperature (DBTT) model, with the ability to turn 


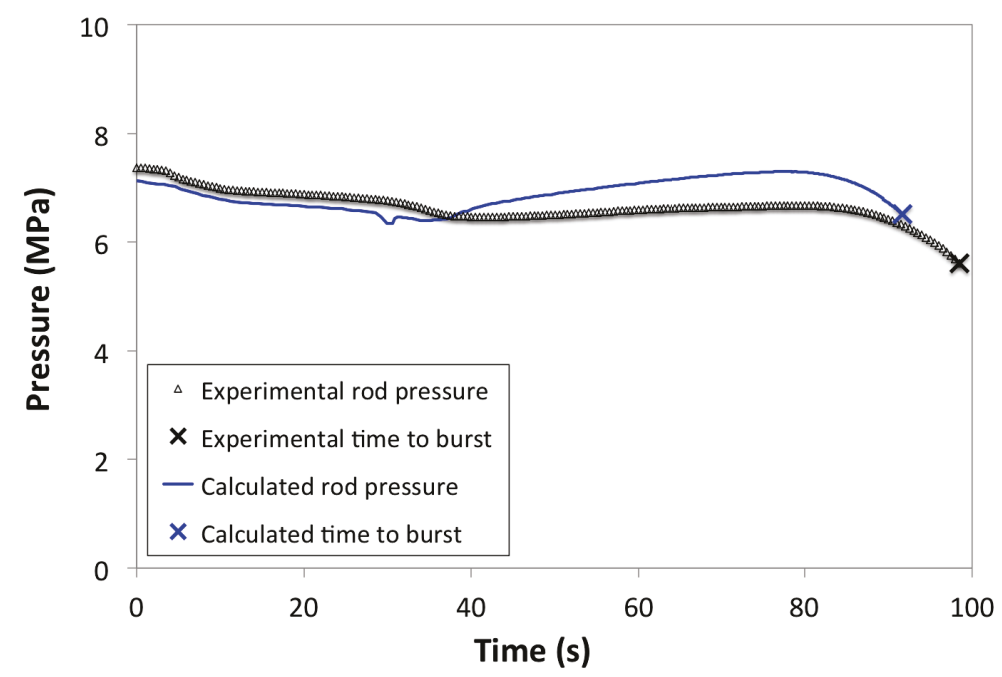

(a)



(b)

Figure 2.4: Comparison between on-line measurements and BISON predictions of fuel rod inner pressure and time to cladding burst for the Halden IFA-650.2 (left) and IFA-650.10 (right) tests. Time zero corresponds to the beginning of the LOCA phase (blowdown). 
off fuel creep and plasticity when the temperature is lower than a transition temperature. Testing has shown that in the low temperature region, creep strains can be negligible while the creep model cannot operate in tandem with the smeared cracking model. Turning off the computation of creep at low temperature improves the numerical convergence while still providing reasonable results. Figure 2.5 shows the fracture strength of ceramic $\mathrm{UO}_{2}$ as a function of temperature. The ductile brittle transition is clearly obvious, and the transition temperature can depend on the grain size and strain rate. A default DBTT is used in the code, and an input parameter can be used to override the default model to represent other material behavior.

- Changes were made to the material parameters in the smeared cracking model to allow adjustment of the fracture energy. The exponential strain softening model in the original code has only one parameter which is used mainly to compute the residual stiffness, and the cracking is found to occur too quickly and can cause numerical issues. A new parameter $\beta$ is added to the model that can delay the cracking process which essentially raises the fracture energy. Though it may predict relatively high stresses after cracking, the numerical robustness can be improved at lower values of $\beta$ in general.

- An option was added to enforce full-shear retention in the elasticity tensor for the smeared cracking model.

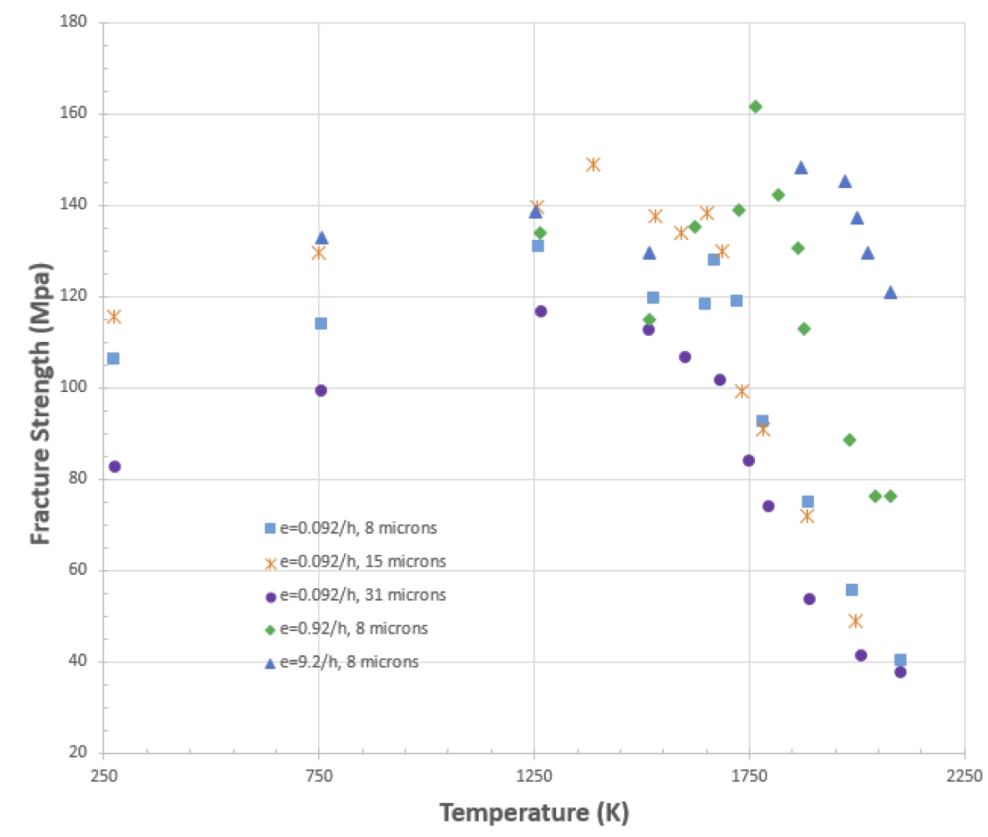

Figure 2.5: Fracture strength of $\mathrm{UO}_{2}$ as a function of temperature. 


\subsubsection{Results}

In a case study of R- $\theta$ geometry with 3-cracking directions, the effect of the material parameter $\beta$ and use of full shear retention method can be seen in Figure 2.6. Running to a terminal power of $40 \mathrm{~kW} / \mathrm{m}$ stands for the case that successful completes; others failed to converge before reaching the peak power. With an assumption of full shear retention, the test case can converge successfully with lower values of $\beta$. Full shear retention with $\beta=0.01$ was used as the default input for subsequent testing cases.

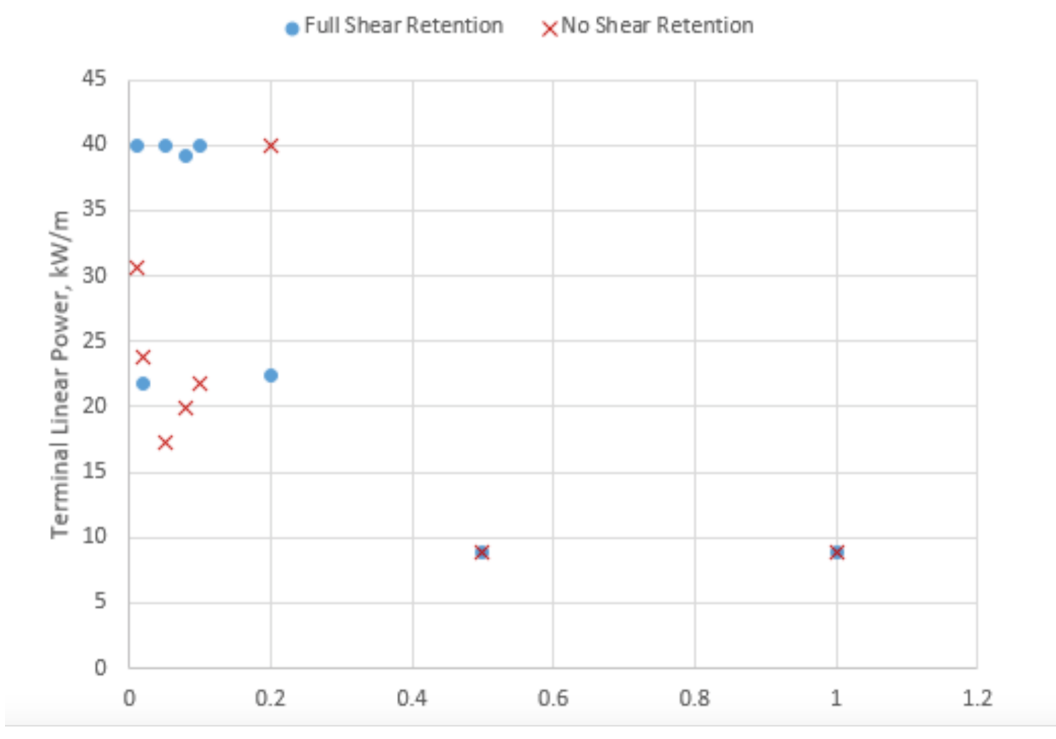

Figure 2.6: Effect of $\beta$ material parameter and shear retention on code convergence.

Notable improvements were made to the convergence of R-Z (radial-axial geometry with axisymmetric element) test cases. An example case on a rodlet problem using linear elements is described here. Key input parameters are provided in Table 2.3 .

The linear power ramps up to $20 \mathrm{~kW} / \mathrm{m}$ and is then held constant; a uniform axial power profile is assumed. All fuel mechanical models are used in this case: relocation, swelling/densification, and creep and hotpressing. This represents the case of analyzing fuel rod behavior under normal operation conditions. A recommended set of BISON input parameters for the smeared cracking model is as follows: cracking_release $=$ exponential, cracking_residual_stress $=0.1$, cracking_beta $=0.01$, and compute_method $=$ ShearRetention. No specific solver is required for running the model, and for this problem, both GMRES method and SuperLU method were tested and provided converged results.

Figure 2.7 provides contours of the hoop and axial crack flags, which show that cracking occurs at the peripheral region of fuel. Figure 2.8 shows the stress evolution at one selected element on the fuel outer surface. The result represents reasonably well the stress changes caused by fuel cracking. 


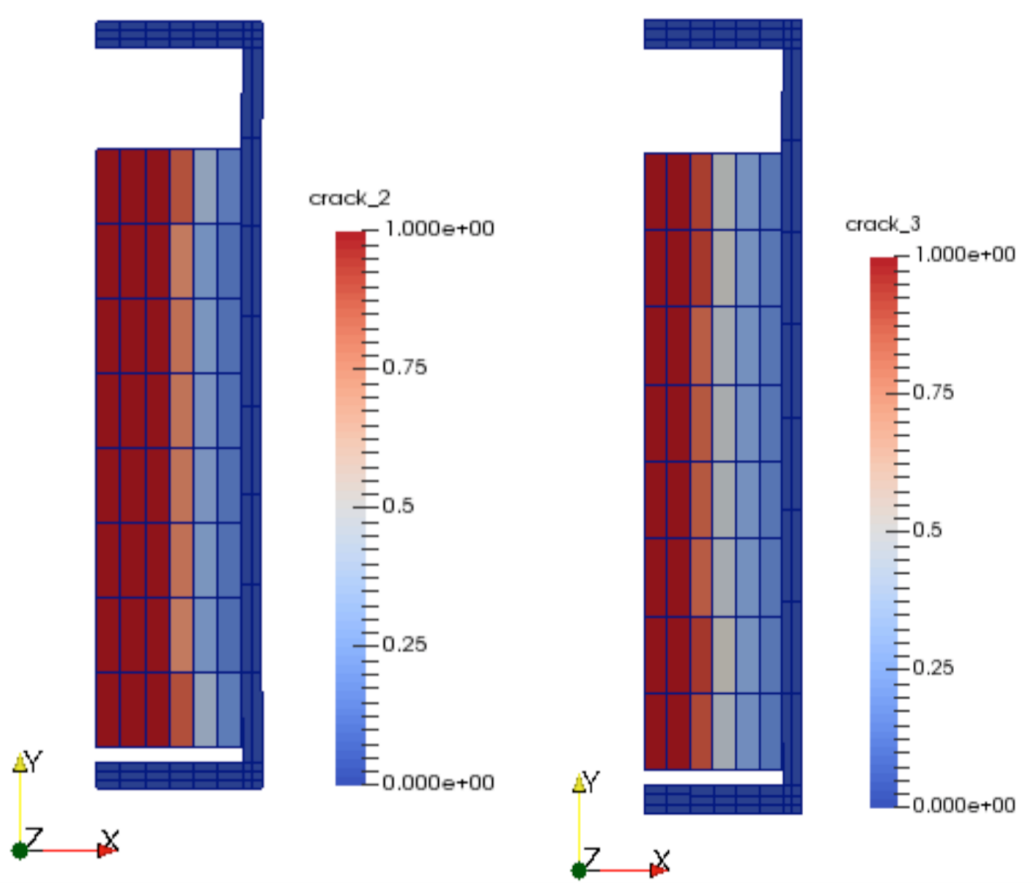

Figure 2.7: Contours of crack flags at the end of simulation (crack_2 represents axial crack; crack_3 represents radial crack); crack flag is defined as the post-cracking stiffness to the original stiffness.

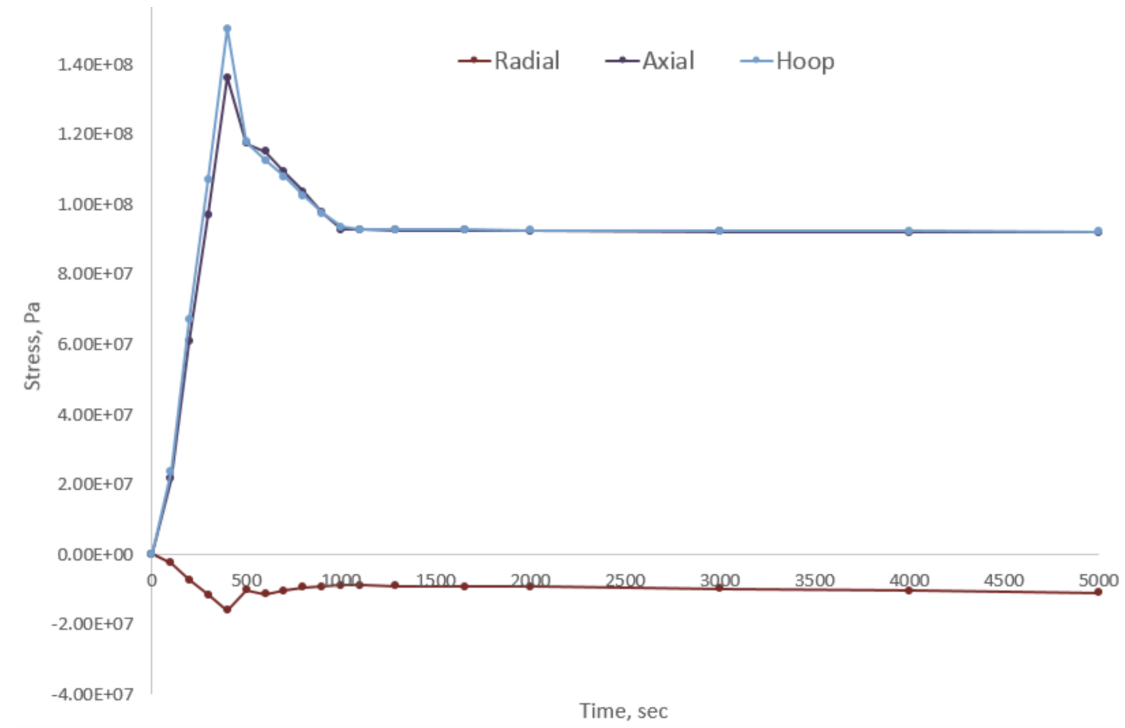

Figure 2.8: Hoop, radial, and axial stresses at a selected element at the peripheral region of fuel pellet near beginning of life. 
Table 2.3: Input parameters for a rodlet problem

\begin{tabular}{lc}
\hline Parameter & Value \\
\hline Fuel pellet diameter $(\mathrm{cm})$ & 0.82 \\
Cladding inner diameter $(\mathrm{cm})$ & 0.836 \\
Cladding outer diameter $(\mathrm{cm})$ & 0.948 \\
Total rod length $(\mathrm{m})$ & 0.128 \\
Fuel stack length $(\mathrm{m})$ & 0.1 \\
Fast neutron flux $\left(\mathrm{n} / \mathrm{m}^{2}-\mathrm{s}\right)$ & $6.0 \mathrm{E}^{18}$ \\
Fill gas & $\mathrm{Helium}^{2}$ \\
Fill gas pressure $(\mathrm{MPa})$ & 2 \\
Coolant pressure $(\mathrm{MPa})$ & 15.5 \\
Fuel density $(\% \mathrm{TD})$ & 95 \\
Fuel elastic modulus $(\mathrm{GPa})$ & 200 \\
Fuel Poisson's ratio & 0.3 \\
\hline
\end{tabular}

\subsubsection{Summary}

The present work improves the numerical robustness of BISON for modeling fuel fracture behavior using a smeared cracking model; improvements were made by changing the material behavior models as well as on numerical methods. It appears that the combination of those changes enable getting converged results for many test cases that used to fail. Notable improvements have been made to the convergence of R-Z (radial-axial axisymmetric) test cases in predicting radial and axial cracks and the convergence of $\mathrm{R}-\boldsymbol{\theta}$ (radial-circumferential) test cases on modeling radial cracks. Test results have shown reasonable prediction of the stress field after fuel cracking. Tests on R-Z geometry in general show better numerical stability and the smeared cracking model can be used in modeling of a fuel rod problem under operation condition in combination with other material/behavior models. 


\section{Additional Major Accomplishments}

\subsection{BISON Validation Summary Article}

FY-16 activities included publication of a summary journal article on BISON LWR validation activities [13]. The paper begins with a brief overview of BISON's computational framework, governing equations, and general material and behavioral models. Both code and solution verification are described. The validation cases considered to date are identified, and specific models and material properties used throughout the validation process are given. Results are consolidated to provide an overall view of how the code is predicting physical behavior. A brief summary of the key results from that article are provided here.

\subsubsection{Verification}

An essential prerequisite to any validation work is code and solution verification. BISON code verification is principally done using an extensive set of code regression tests, that are exercised every time a modification is proposed to the code. The structure and several examples of the BISON code verification process are given in [14].

Solution verification is also essential, to demonstrate that numerical solutions have adequate spatial and temporal resolution for the set of validation problems considered. To demonstrate this a prototypical validation problem was constructed using the set of material and behavior models employed in the validation cases, including boundary conditions and a typical power history. The problem was then run using a series of computational meshes and time increments to observe numerical convergence. Results from the spatial resolution study are shown in Figure 3.1, where relative percent error is presented for four metrics of interest: Power, Fuel Centerline Temperature (FCT), Fission Gas Release (FGR), and Rod diameter. Results are given as a function of the total number of radial elements in the mesh, where the relative error is with respect to the finest mesh considered. All LWR validation problems were run using the finest mesh shown in the figure demonstrating adequate mesh resolution for the validation study. Similar results are provided in the manuscript for temporal numerical resolution.

\subsubsection{Experimental data used for validation}

Table 3.1 summarizes the experimental fuel rods that were used in the paper and represented the set of cases that had been considered at the time the article was prepared. Measured quantities include fuel centerline temperature (FCT), fission gas release (FGR), and cladding outer diameter (Rod Dia) both before and following fuel-clad mechanical contact. Many of these integral rod experiments were chosen based on INL participation in the IAEA sponsored FUMEX-III 


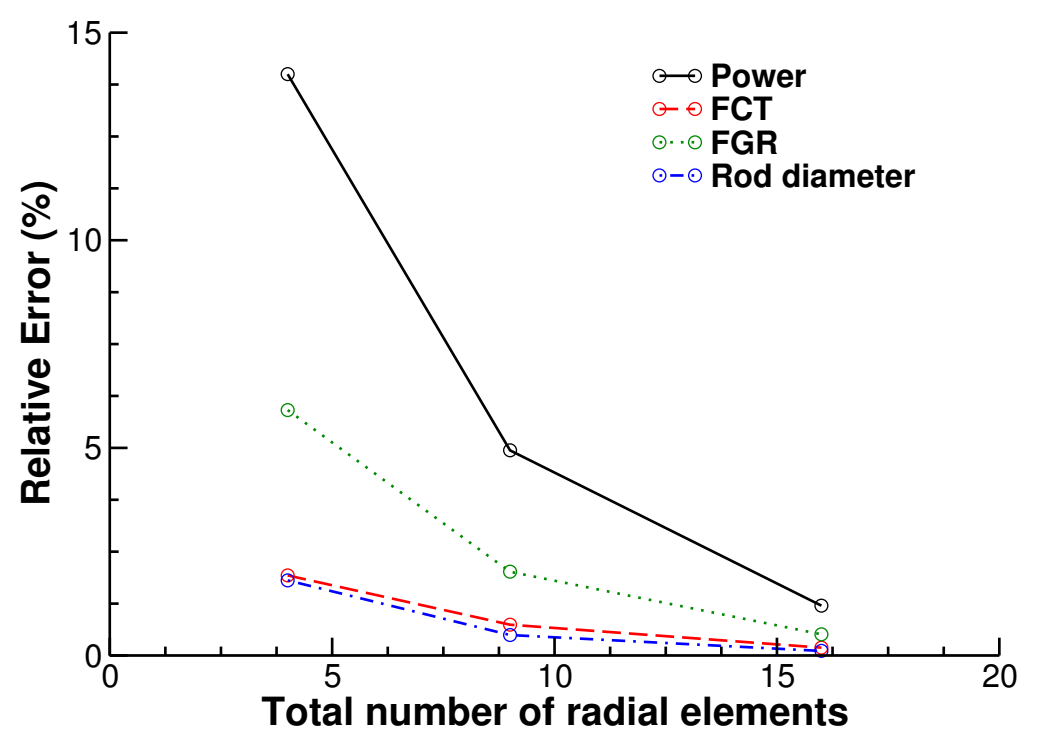

Figure 3.1: Spatial resolution results for the solution verification study. Relative percent error is presented for four metrics of interest: Power, FCT, FGR, and clad diameter as function of the total number of radial elements in the mesh. The relative error is with respect to the finest mesh considered.

Coordinated Research Project and are priority cases from either FUMEX-II [15] or FUMEXIII [16].

\subsubsection{Thermal Behavior}

Accurate fuel temperature prediction is essential for a fuel performance code as temperature is important for assessing fuel rod performance and safety. Additionally, many other important physical phenomena depend highly on temperature.

Temperature comparisons during the first rise to power are significant as they isolate several important aspects of fuel rod behavior before complexities associated with higher burnups are encountered. Accurate prediction of beginning of life (BOL) fuel centerline temperature requires accurate models for the fuel and cladding thermal conductivity and gap conductance. Figure 3.2 summarizes BOL fuel centerline temperature comparisons for the set of experiments in Table 3.1 where such data are available. Plotted is the predicted versus measured fuel centerline temperature as the rod power is increased during power-up. For all cases considered to date, deviations between BISON predictions and experimental data are less than $\pm 10 \%$.

Temperature comparisons during long irradiations show BISON's ability to account for changing fuel thermal conductivity and fuel-clad gap behavior. Comparisons in the paper are given in terms of four different burnup increments: $0 \leq B u<20,20 \leq B u<40,40 \leq B u<60$, and $B u \geq 60 \mathrm{MWd} / \mathrm{kgUO}_{2}$. As an example, temperature comparisons for the interval $20 \leq B u<40$ are shown in Figure 3.3. Although there is slightly more scatter than for the beginning of life comparisons in Figure 3.2, comparisons at higher burnup are very reasonable. 
Table 3.1: Overview of the main integral experimental data used for validation of BISON.

\begin{tabular}{|c|c|c|c|c|c|}
\hline Experiment & Rod & FCT & FGR & $\begin{array}{c}\text { Rod } \\
\text { Dia }\end{array}$ & Ref \\
\hline IFA-431 & $1,2,3$ & $\mathrm{X}$ & & & 17 \\
\hline IFA-432 & $1,2,3$ & $\mathrm{X}$ & & & [17. 18 \\
\hline IFA-515.10 & A1 & $\mathrm{X}$ & & & 19 \\
\hline IFA-597.3 & 8 & $\mathrm{X}$ & $\mathrm{X}$ & & 18 \\
\hline Ris $\varnothing-3$ & AN3,AN4 & $\mathrm{X}$ & $\mathrm{X}$ & & 18 \\
\hline Ris $\emptyset-3$ & AN2 & & $\mathrm{X}$ & $\mathrm{X}$ & {$[18$} \\
\hline Ris $\varnothing-3$ & GE7 & & $\mathrm{X}$ & $\mathrm{X}$ & 18 \\
\hline Ris $\emptyset-3$ & II3 & $\mathrm{X}$ & $\mathrm{X}$ & $\mathrm{X}$ & 18 \\
\hline Ris $\emptyset-2$ & GE-m & & $\mathrm{X}$ & $\mathrm{X}$ & 18 \\
\hline OSIRIS & J12 & & $\mathrm{X}$ & $\mathrm{X}$ & 18 \\
\hline REGATE & & & $\mathrm{X}$ & $\mathrm{X}$ & 18 \\
\hline USPWR 16x16 & TSQ002,TSQ022 & & $\mathrm{X}$ & $\mathrm{X}$ & 18 \\
\hline IFA-431 (3D) & 4 & $\mathrm{X}$ & & & [17. 20 \\
\hline R.E. Ginna & 2,4 & & $\mathrm{X}$ & $\mathrm{X}$ & {$[18$} \\
\hline OSIRIS & H09 & & $\mathrm{X}$ & $\mathrm{X}$ & 18 \\
\hline HBEP & BK363,BK365 & & $\mathrm{X}$ & & 15 \\
\hline IFA-534 & 18,19 & & $\mathrm{X}$ & & 18 \\
\hline IFA-535 & 809,810 & & $\mathrm{X}$ & & 18 \\
\hline IFA-562.2 & $15,16,17$ & $\mathrm{X}$ & $\mathrm{X}$ & & 21 \\
\hline Ris $\emptyset-3$ & II5 & $\mathrm{X}$ & $\mathrm{X}$ & $\mathrm{X}$ & 18 \\
\hline Tribulation & $\mathrm{BN} 1 / 3, \mathrm{BN} 1 / 4, \mathrm{BN} 3 / 15$ & & $\mathrm{X}$ & $\mathrm{X}$ & 18 \\
\hline
\end{tabular}

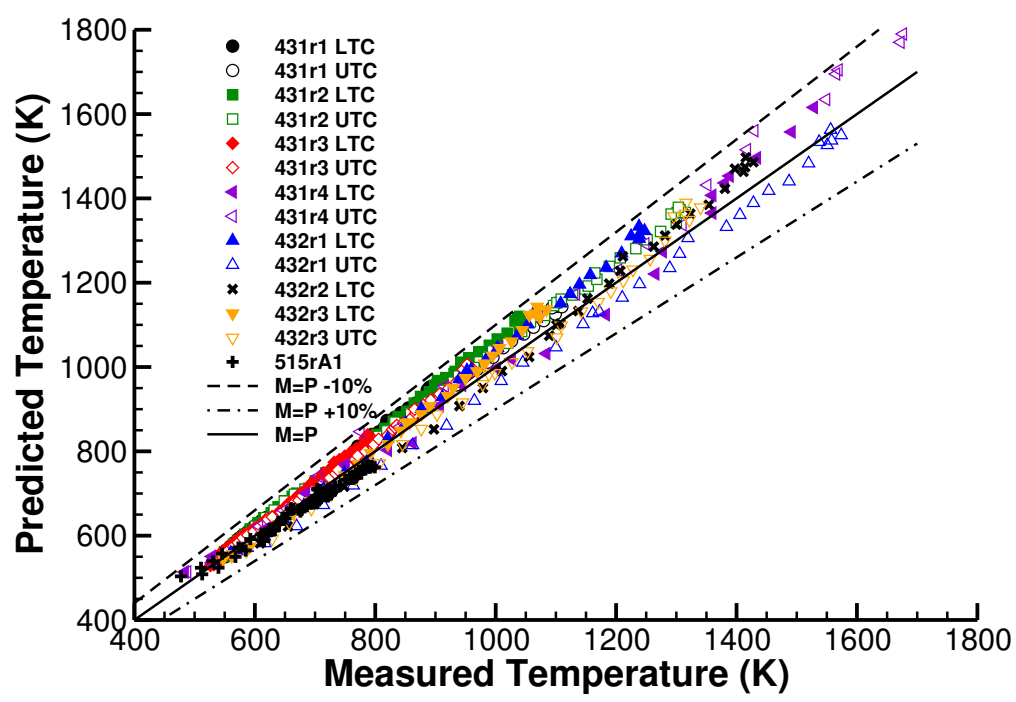

Figure 3.2: BOL measured vs. predicted fuel centerline temperature for fuel rods in IFA-431, IFA-432, and IFA-515.10. LTC and UTC stand for lower and upper thermocouple measurements, respectively.

Five of the experiments listed in Table 3.1 (Risø-3 rods AN3, AN4, II3, II5 and IFA-597.3 rod 8) include measurements of fuel centerline temperature during power ramps following base 


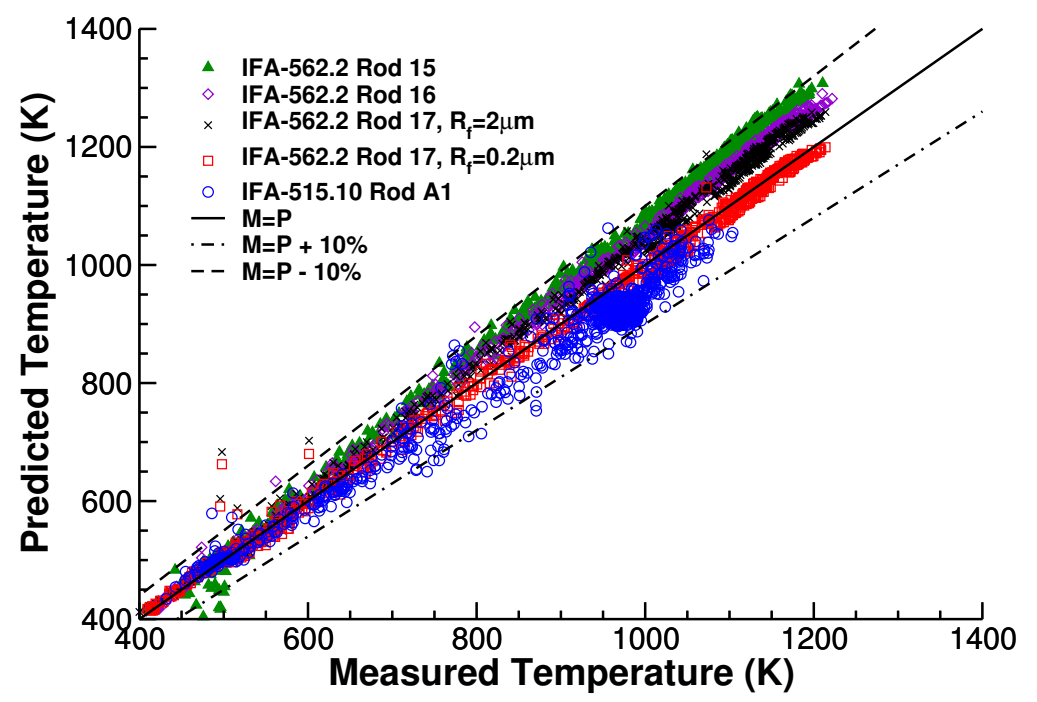

Figure 3.3: Comparison of the measured vs. predicted fuel centerline temperature for through life rods for the burnup range: $20 \leq B u<40$. The $\mathrm{R}_{f}$ parameter in the IFA-562.2 Rod 17 series labels indicate the fuel roughness used in the simulation.

irradiation. These experiments include rods with burnups ranging from 14.5 to $61.6 \mathrm{MWd} / \mathrm{kgU}$. In all cases, experimental rods were refabricated for installation in the test reactor by shortening mother rods. Similar to Figs. 3.2 and 3.3, Fig. 3.4 compares measured and predicted fuel centerline temperatures for the five ramp test experiments. Comparisons are reasonable, however, in contrast to the prior comparisons, some points fall outside the $\pm 10 \%$ difference bands. Certainly approximations involved in simulating the rod refabrication process add uncertainty to the temperature predictions.

\subsubsection{Fission Gas Release}

BISON predictions of integral fuel rod FGR from simulations of the validation cases listed in Table 3.1 have been compared to the available experimental data. Due to the inherent uncertainties of fission gas behavior modeling, a deviation of fuel performance code predictions from the experimental data within a factor of about 2 up and down is generally regarded as satisfactory [15, 16, 22, 23]. A recent uncertainty evaluation study supported the estimation of a factor of 2 as range of tolerance for high calculated FGR values, yet pointing out that higher deviations may be expected for calculated FGR around 10\% and lower [24]. The comparison of BISON calculations and experimental data of integral FGR at the fuel rod end-of-life (EOL) is summarized in Figure 3.5. BISON accuracy in predicting FGR appears to be consistent with state-of-the-art modeling [15, 16, 22] and with the involved uncertainties.

\subsubsection{Rod Diameter}

Accurate simulation of the mechanical behavior of fuel rods is important when attempting to make predictions about cladding structural integrity, for example as a result of pellet cladding 


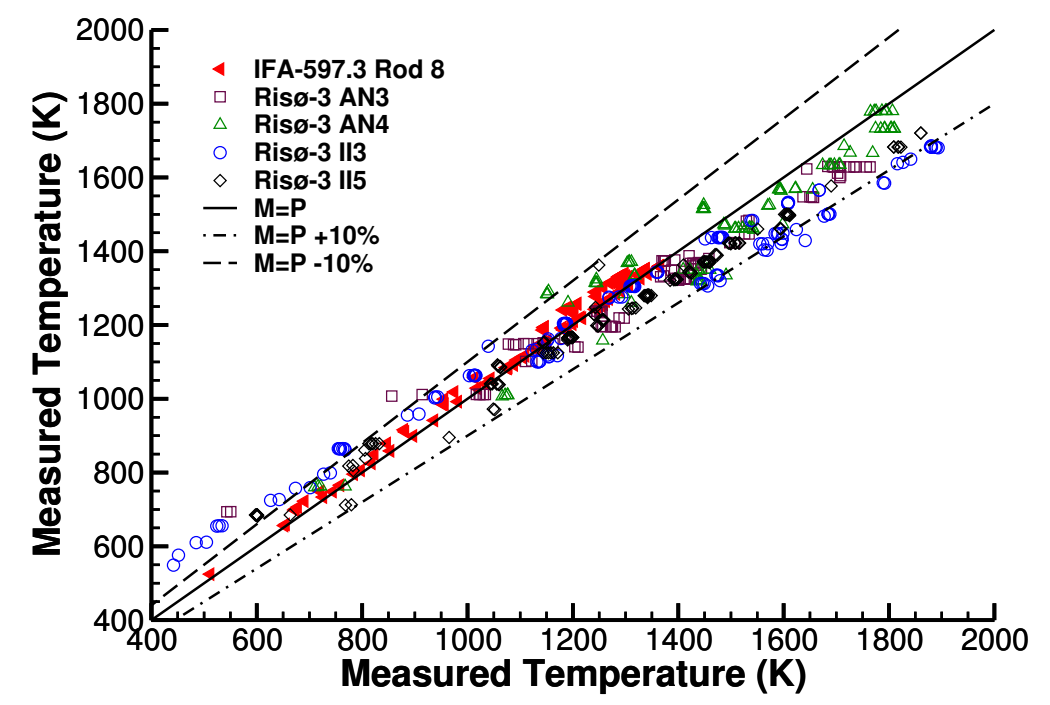

Figure 3.4: Comparison of the measured vs. predicted fuel centerline temperature for fuel rods that experienced power ramps.

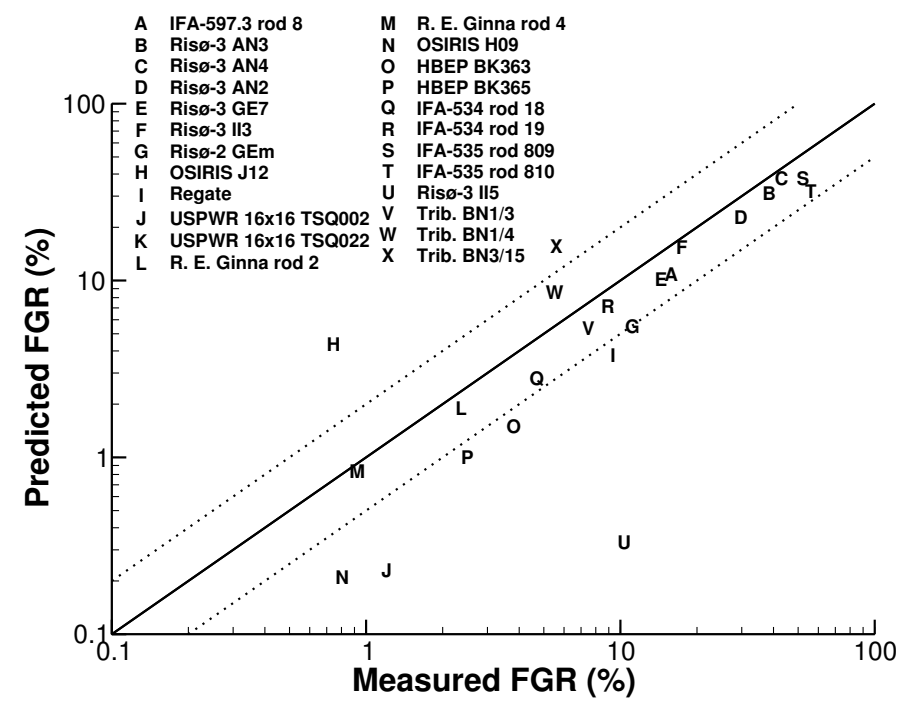

Figure 3.5: Fission gas release measured versus predicted comparisons with a factor of 2 error bands.

mechanical interaction (PCMI). Eleven of the experiments listed in Table 3.1 included rod diameter measurements. All were made post irradiation in a hot cell, with a few including measurements following both base irradiation and after a power ramp.

Table 3.2 summarizes the rod diameter comparisons following base irradiation, in terms of the difference between the measured and predicted values. As indicated in the table, comparisons are made either at the rod axial midplane or averaged over the rod length, based on the available experimental data. The comparisons are separated by cladding type (Zircaloy 4 and Zircaloy 2) 
and ordered according to the final burnup. The comparison results of Table 3.1 are plotted in Figure 3.6 in terms of diameter change vs burnup. In general, the results indicate a tendency to underpredict the diameter change early in life, and more significantly overpredict the diameter change late in life. The early life comparisons typically occur before fuel-clad contact, when clad deformation is dominated by clad creep down; comparisons thus indicate a tendency to overpredict clad creep rates. Although the number of low burnup points are limited, comparisons thus far indicate this overprediction is more severe for Zircaloy-2 than for Zircaloy-4. At higher burnups, following fuel-clad contact, the clad diameter increase is controlled by the mechanical behavior of the fuel. The fact that the clad diameter change (measured - predicted) is significantly negative indicates a strong tendency to overpredict the fuel radial growth. BISON's over prediction of final rod diameter may be partially due to its lack of a relocation recovery model, which would recover a portion of the relocation strain upon fuel-cladding contact. Uncertainty in the initial fuel porosity and densification during irradiation provide further explanation for poor diameter comparisons. Fuel creep, which has been neglected in the present paper, is also expected to play a significant role.

To explore some of these issues a simple parametric study was performed for a single validation case (R. E. Ginna Rod 2) with three separate modifications to the base calculation: 1) fuel relocation was deactivated, 2) fuel relocation was active but relocation strains were reduced by $50 \%$ and, 3) fuel relocation was active but fuel densification was increased by $0.5 \%$. The partial reduction in relocation strain was motivated by the relocation recovery model in the FRAPCON fuel performance code, which recovers (by default) $50 \%$ of the relocation strain upon contact [25]. Referring to the diameter change (measured - predicted) parameter in Table 4, which was $-71.8 \mu \mathrm{m}$ for the Ginna Rod 2 case, simply removing relocation strain from the calculation dropped that difference to $-19.6 \mu \mathrm{m}$, demonstrating that the BISON relocation model, which is simplistic and highly empirical, has a substantial effect on the rod diameter comparison. Recovering half of the relocation strain resulted in a final clad diameter change of -45 $\mu \mathrm{m}$, demonstrating the importance of including relocation recovery behavior. Simply increasing the fuel densification by $0.5 \%$ resulted in a final diameter change of $-60.1 \mu \mathrm{m}$, indicating that uncertainties in this parameter will have a smaller, but still significant effect.

Ongoing PCMI validation efforts will focus on the BISON fuel creep, relocation and densification models. It is worth noting that prediction of cladding diametral strain is in general a difficult area for fuel performance codes, as demonstrated by international benchmark exercises [15, 16].

\subsubsection{Summary}

BISON predictions have been compared to a wide variety of LWR experiments. Results indicate:

- Temperature comparisons at beginning of life, during irradiation to high burnup, and during power ramps, are all very reasonable.

- Accuracy in predicting FGR appears to be consistent with state-of-the-art modeling and with the involved uncertainties. 
Table 3.2: Base irradiation

\begin{tabular}{|c|c|c|c|c|}
\hline Case Description & $\begin{array}{l}\text { Clad } \\
\text { Type }\end{array}$ & $\begin{array}{l}\text { Axial } \\
\text { Comparison } \\
\text { Location }\end{array}$ & $\begin{array}{l}\text { Rod Average } \\
\text { Burnup } \\
(\mathrm{MWd} / \mathrm{kgU})\end{array}$ & $\begin{array}{c}\text { Diameter Change } \\
\text { (Meas. - Pred.) } \\
(\mu m)\end{array}$ \\
\hline Tribulation BN1/4 & Zry-4 & Midplane & 19.7 & 16.4 \\
\hline Tribulation BN1/3 & Zry-4 & Midplane & 20.2 & 7.1 \\
\hline OSIRIS J12 & Zry-4 & Average & 23.9 & -18.1 \\
\hline Risø-3 AN2 & Zry-4 & Average & 31.4 & -16.6 \\
\hline Tribulation BN3/15 & Zry-4 & Midplane & 37.7 & -71.1 \\
\hline OSIRIS H09 & Zry-4 & Average & 46.1 & -42.9 \\
\hline REGATE & Zry-4 & Average & 47.0 & 6.5 \\
\hline Tribulation BN1/4 & Zry-4 & Midplane & 50.6 & -53.2 \\
\hline Tribulation BN1/3 & Zry-4 & Midplane & 50.7 & -70.8 \\
\hline Tribulation BN3/15 & Zry-4 & Midplane & 51.1 & -84.8 \\
\hline R. E. Ginna Rod 2 & Zry-4 & Average & 51.2 & -71.8 \\
\hline R. E. Ginna Rod 4 & Zry-4 & Average & 51.2 & -69.2 \\
\hline USPWR TSQ002 & Zry-4 & Average & 53.2 & -26.3 \\
\hline USPWR TSQ022 & Zry-4 & Average & 58.1 & -40.4 \\
\hline Ris $\varnothing-2$ GE-m & Zry-2 & Average & 15.5 & 35.8 \\
\hline Ris $\emptyset-3$ II3 & Zry-2 & Average & 16.4 & 32.8 \\
\hline Ris $\varnothing-3$ GE7 & Zry-2 & Average & 31.4 & 34.1 \\
\hline Ris $\varnothing-3$ II5 & Zry-2 & Midplane & 39.0 & -78.8 \\
\hline
\end{tabular}

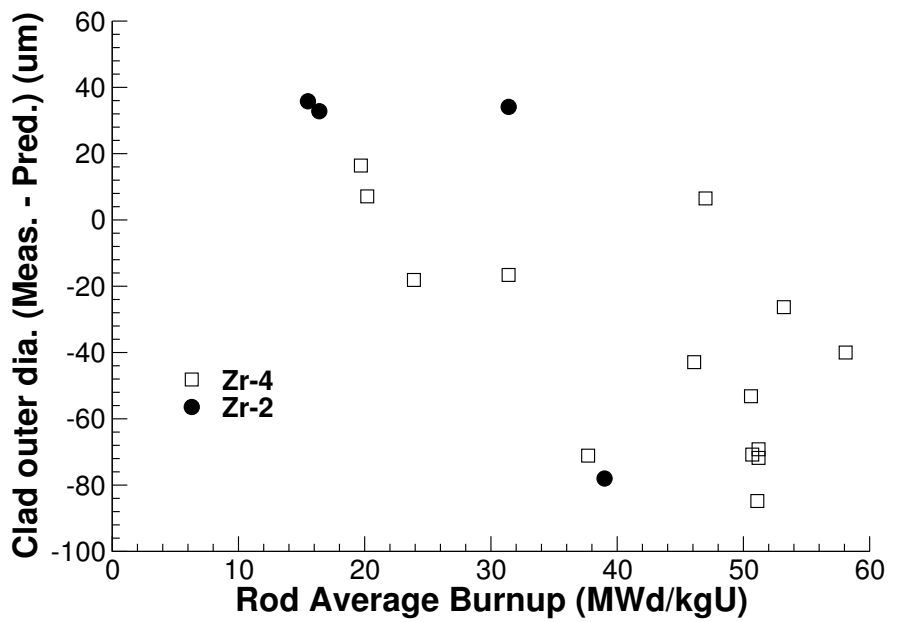

Figure 3.6: The difference between measured and predicted cladding outer diameter as a function of burnup.

- Comparison of rod diameter indicate a tendency to underpredict diameter change early in life, and more significantly overpredict diameter change late in life. Initial rod diameter comparisons are not satisfactory and have lead to consideration of additional separate effects experiments to better understand and predict clad and fuel mechanical behavior.

Results from this study are being used to define and prioritize future code development and validation activities. High priority items include:

- An increased emphasis on separate effects validation experiments, especially for fuel 
swelling and fuel and cladding creep.

- Inclusion of more realistic mechanical models for oxide fuel, specifically smeared cracking and creep.

- Addition of a relocation recovery model.

- Consideration of discrete pellet geometry in validation cases, especially for cladding diameter comparisons.

- Expansion of the validation base to include other fuel types including MOX and Gd-doped fuels.

- Inclusion of the effects of high burnup structure on fuel performance.

- Addition of accident behavior cases (both LOCA and RIA) to the validation base.

\subsection{Missing Pellet Surface Article}

In FY16 a major effort on 3D Missing Pellet Surface (MPS) analysis in BWR fuel was completed and published. The following is a summary of that article [26], which was published in July 2016.

\subsubsection{Introduction}

The metallic cladding that encases light water reactor (LWR) fuel serves as a barrier against release of fission products. Pellet-cladding interaction (PCI), which can be caused by a combination of mechanical interactions between fuel and cladding and chemical reactions, is one of the major causes of cladding failure [27]. The cladding stresses induced by the mechanical PCI are strongly influenced by local pellet geometry. A type of defect, known as a missing pellet surface (MPS) in which a portion of the side of a pellet has been removed due to accidental machining or chipping, has been identified as a significant cause of cladding failure [27, 28] due to its local effects on pellet geometry.

Because of the nature of the defect geometry, modeling the effects of an MPS defect requires a local three-dimensional (3D) model of the defect region, but the model must also incorporate the effects of the full fuel rod. This paper presents an approach to couple a two-dimensional (2D) model of the full rod with a local 3D model of the defective pellet region using the BISON code and a demonstration of this approach on boiling water reactor (BWR) fuel subjected to a local rapid increase in power due to a control blade withdrawal.

\subsubsection{Models}

The thermo-mechanical response of LWR nuclear fuel in the reactor environment is affected strongly by the composition of the fill gas, which evolves during the life of the rod because of the release of gaseous fission products into the plenum gas, which is initially helium. To accurately represent the response of a local region of a fuel rod, such as the region adjacent to 
an MPS defect, one must account for the response of the entire rod. To account for this, two separate models are employed: a 2D axisymmetric finite element model of a full fuel rod (shown in Figure 3.7[a]), and local models of the region of interest (shown in Figures 3.7 b,c]).

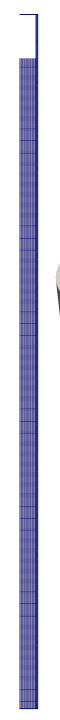

(a)

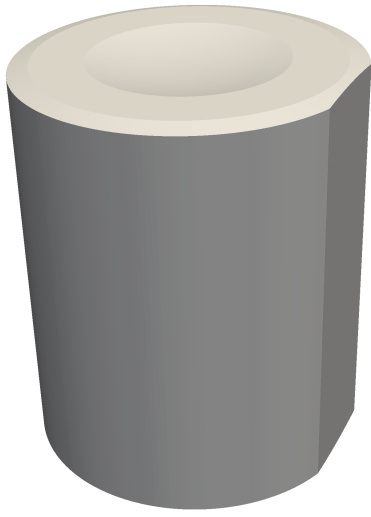

(b)

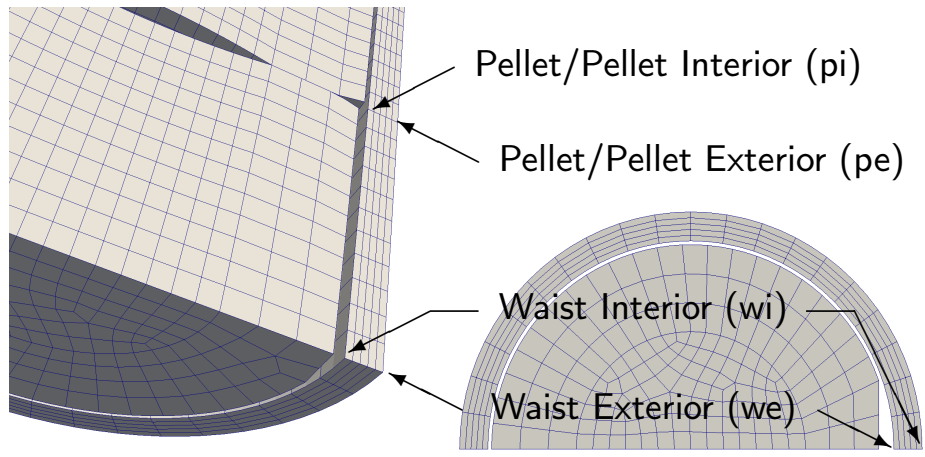

(c)

Figure 3.7: Models employed in this work: (a) 2D axisymmetric model of a full rod; (b) 3D representation of a pellet with an MPS defect; (c) zoomed-in views of 3D and 2D models of fuel and cladding in the vicinity of the MPS defect.

The full rod model and the local model are both run through the full irradiation history. A one-way coupling strategy is employed where the time histories of the total fission gas released, total plenum volume, and plenum gas temperature are transferred from the full rod model to the local model. This set of data is sufficient to fully define the pressure boundary conditions and plenum gas thermal conductivity needed in the local model.

This coupling strategy is very flexible and allows the combined global and local models to represent the effects of transients that affect primarily local regions of the rod and transients that are more global in nature. This has been demonstrated recently on an analysis of BWR fuel [26] for which excerpts are summarized in the following:

Control blades are employed in BWRs to control the power. During the course of operation, these control blades are withdrawn in small increments to adjust the power. These small adjustments have a minimal impact on the global response of the full fuel rod, but result in a significant and rapid local increase in the power in the vicinity of the tip of the control blade.

The proposed coupling strategy was employed to represent a BWR fuel rod containing an MPS defect irradiated for one cycle in a position away from the influence of a control blade and then moved to a position adjacent to a control blade. Power at the MPS defect location is initially suppressed due to the control blade, but the power significantly increases as the blade is withdrawn a small amount. The power is then held at that level, until the power in the full rod is 
increased by $50 \%$ to simulate a ramp to high power. While the control blade withdrawal event has a negligible effect on fission gas, the high power ramp causes a significant increase in fission gas release and a corresponding decrease in fill gas thermal conductivity.

\subsubsection{Results}

Figure 3.8 shows contours of hoop stress and temperature in the cladding from the 3D local model of the MPS defect region. The stresses are significantly affected by the defect in this region because of the plate bending behavior of the cladding as it spans the gap created by the defect. Cladding temperatures are lower in that region due to decreased conductance.

The local defect region was modeled using both a 3D model and a 2D generalized plane strain model (Figure 3.7[c]) to demonstrate the effects of modeling assumptions. Figure 3.9 shows time histories of quantities of interest in the cladding at selected location at cladding interior adjacent to the pellet/pellet interface and pellet waist, as denoted in Figures 3.7[c].

The models with a defect have increased stresses and creep strains compared to the baseline case at all locations considered here. The 2D model reasonably replicates the response of the 3D model at the pellet waist, but differs significantly from the response at the pellet/pellet interface.

\subsection{Halden 3D MPS Experiment}

An in-reactor experiment is being designed to validate the pellet-cladding mechanical interaction (PCMI) behavior of the BISON fuel performance code. The experimental parameters for the test rod to be placed in the Halden Research Reactor are being determined using BISON simulations. The fuel rod includes a missing pellet surface (MPS) defect to generate large local cladding deformations, which will be measurable after typical burnup levels.

\subsubsection{Introduction}

There is considerable interest in modeling PCMI such as that induced by MPS defects [29-31]. Because of its 3D capabilities, BISON is uniquely positioned to model this phenomenon [1], and it has been employed recently for this purpose [26]. There are significant efforts underway to validate BISON [13], and having validation data for 3D PCMI scenarios would improve confidence in predictive capabilities in this area. An opportunity arose recently to utilize an experiment in the Halden Research Reactor to generate data for validating 3D simulations of the behavior of MPS defects. This experiment will involve irradiation of two fuel rods, both including MPS defects, in the Halden Research Reactor. One of the two rods (reference rod) will be instrumented with two fuel centerline thermocouples (TC) for the fuel temperature measurements to be compared to BISON calculations. In addition to temperature measurements, periodic examinations will include cladding diameter measurements during the experiment. The reference rod has been modeled initially and will be briefly described here.

The primary objectives of the BISON simulations in this phase of the experimental planning are to ensure that the effects of MPS defects on cladding deformation will be detectable during the experiment, and to ensure that thermocouple temperatures are below acceptable limits. 


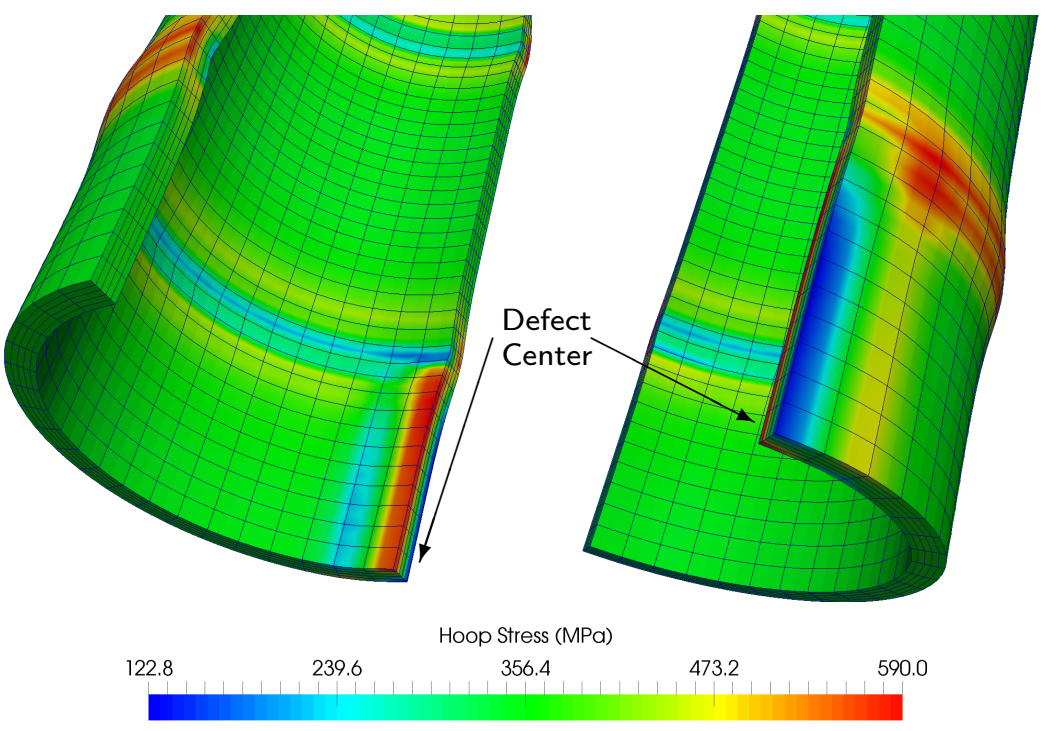

(a)

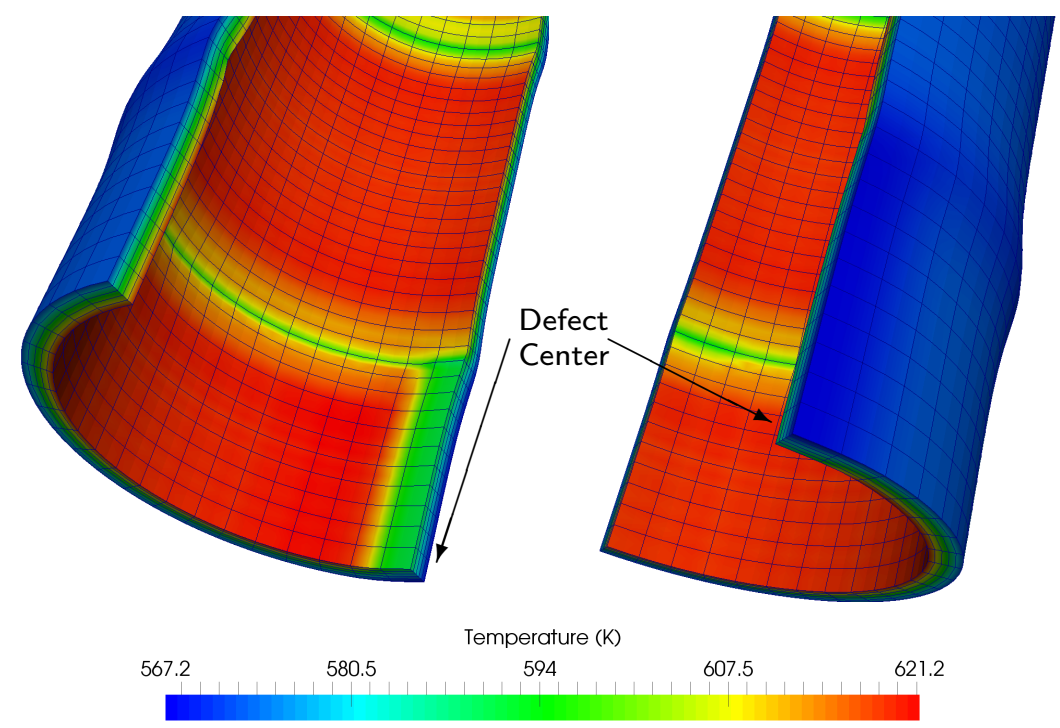

(b)

Figure 3.8: Contours of (a) hoop stress and (b) temperature in the cladding for the 3D model of a $0.1-\mathrm{mm}$ deep defect at the end of the power history applied here. Two views of the same model are shown in each case. 

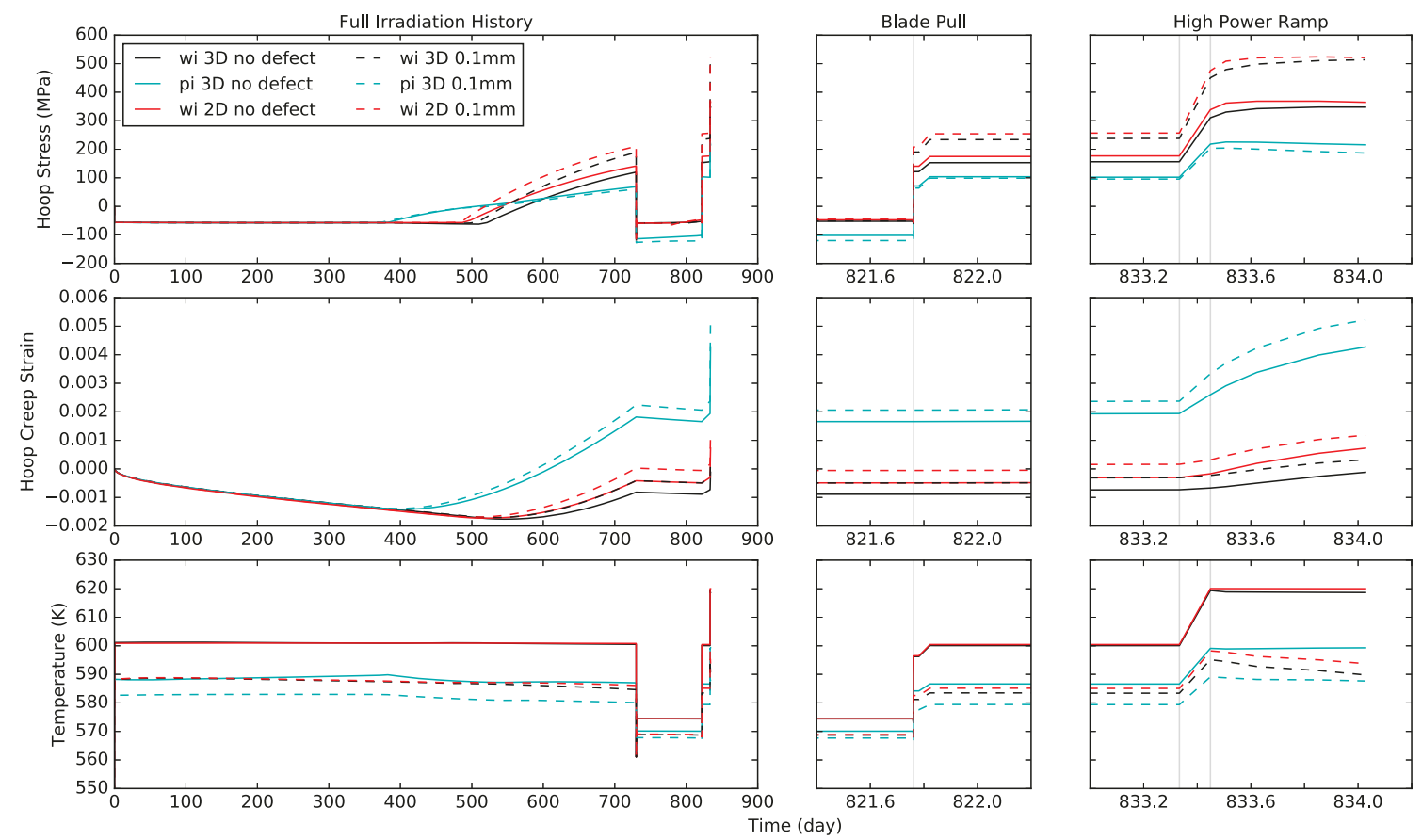

Figure 3.9: Time history of response at locations of interest denoted in Figure 3.7 [c]. Results are shown for $2 \mathrm{D}$ and $3 \mathrm{D}$ models of the defective pellet region for no defect and a $0.1-\mathrm{mm}$ defect.

\subsubsection{Fuel Rod Configuration}

The fuel rod will comprise of an approximately $200 \mathrm{~mm}$ long stack of $\mathrm{UO}_{2}$ fuel pellets and Zircaloy-4 cladding with $\mathrm{He}$ as the fill gas. The rod will have a narrow initial pellet/clad gap to promote PCMI early in the experiment and enhance the effect of the MPS on cladding deformation. The irradiation will be performed under typical PWR conditions for roughly 2 years.

A schematic of the fuel and cladding geometry is shown in Figure 3.10. The rod will include two MPS pellets. One of these will be a hollow pellet located in the upper part of the rod, and the other will be a solid pellet located at the fuel mid-plane. One centerline TC will be located in correspondence of the upper MPS pellet, while the other will be located in a non-defective pellet at a symmetric, lower axial position relative to the upper TC. Because the axial power profile will be approximately flat, the lower TC will serve as reference to assess the effect of MPS on fuel temperature through comparison with the upper TC signal.

\subsubsection{Models}

Several 3D models were created to represent the various fuel rod configurations proposed for the Halden experiment. Initially, since the number of pellets and MPS locations had not been finalized, a simplified 5-pellet model (Figure 3.11) was used to perform preliminary simulations. This model consists of both solid and annular pellets with an MPS defect in the central pellet. 


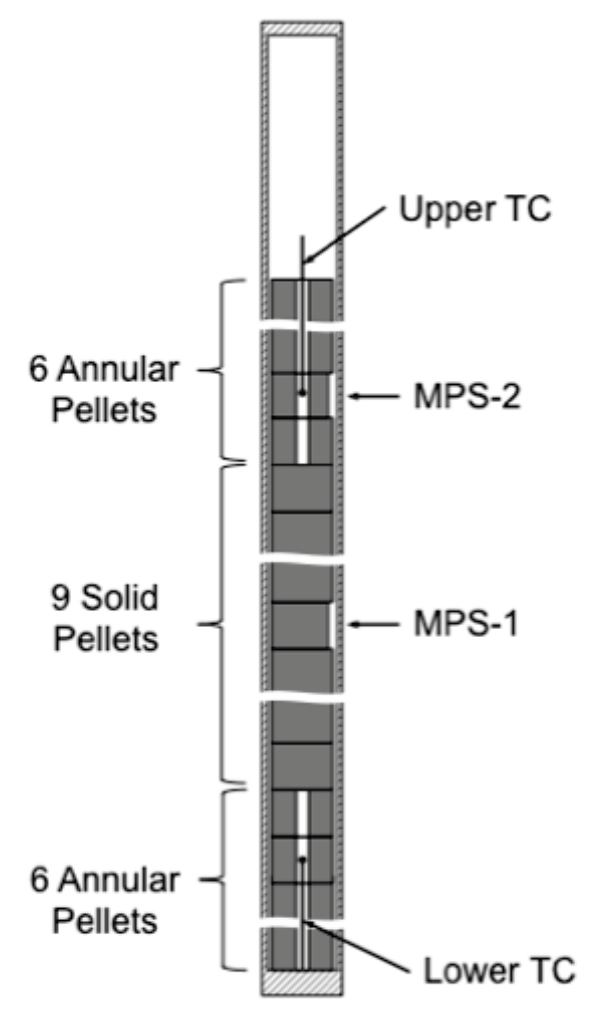

Figure 3.10: Halden fuel rod schematic.

The MPS depth in this model was varied from $0.1 \mathrm{~mm}$ to $0.3 \mathrm{~mm}$. Once the experimental configuration of the fuel rods became more certain, a full-length 3D model containing all 21 pellets was developed based on the schematic shown in Figure 3.10 .

\subsubsection{MPS Defect Depth Effect}

The effect of MPS depth on cladding deformation is a primary concern in determining the fuel rod geometry. Figure 3.12 shows the radial deformation of the cladding plotted as a function of distance from the bottom of the fuel rod for a series of MPS depths using the simplified 5-pellet model. The plot shows that even with the smallest MPS depth of $0.1 \mathrm{~mm}$, the radial deformation at the end of the 2-year experiment is approximately 20 microns. Since the resolution of the device for measuring cladding displacement is a few microns, the cladding deformation induced by the $0.1 \mathrm{~mm}$ MPS defect should be easily detectable at the end of the experiment. Such MPS depth is representative of defects of practical interest. However, introducing a deeper MPS defect in one of the two rods in order to provide detectable effects earlier in the experiment and a more extensive database for BISON validation is being considered. 


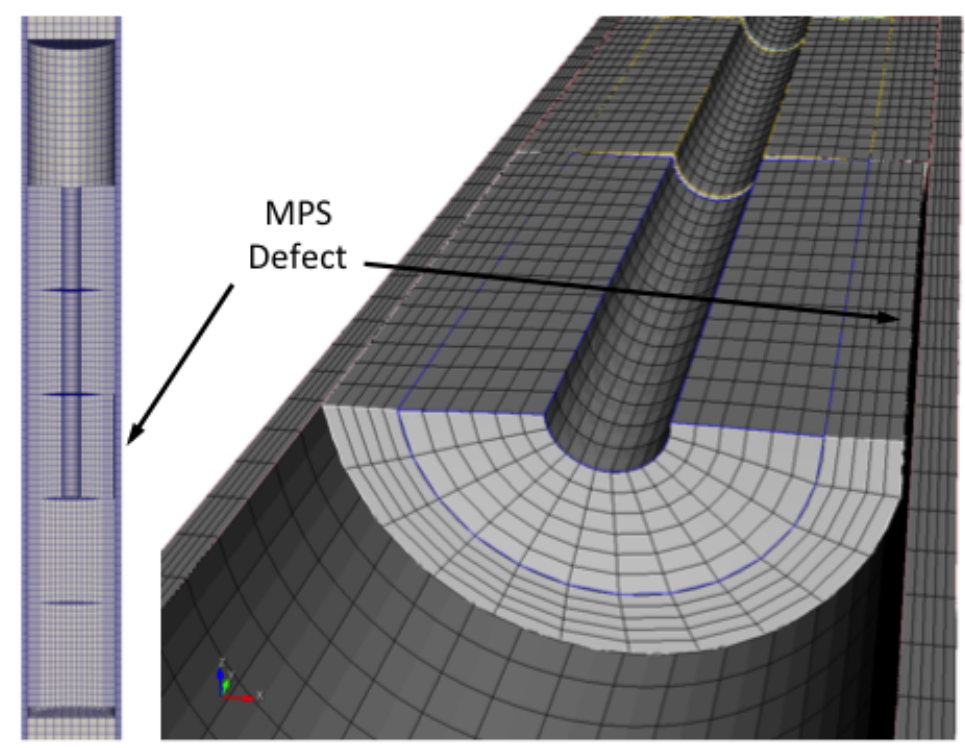

Figure 3.11: 5-pellet BISON MPS defect model.

\subsubsection{Contact Model Comparison}

The contact model used for the pellet-cladding interface has a significant effect on the behavior of a fuel rod simulation. The cladding deformation results shown in Figure 3.12 were calculated using a frictionless contact model for the pellet-cladding interface. For comparison, this interface was also modeled using a glued contact condition, which may be closer to the experimental conditions, but is still an approximation. The glued contact is enforced as soon as initial contact occurs and the interfaces are not allowed to move tangentially or separate under tension. During the intermittent measurements of the cladding deformation, some fuel-cladding separation may occur which will not be accounted for using this contact model.

The differences in the local effects caused by different contact models can be seen in Figure 3.13. The figure shows a contour plot of the effective cladding creep strain in the central MPS defect area. The glued model shows higher local strains near the defect whereas the frictionless model allows the deformation to be distributed along the cladding and thus is less localized. In addition, the plot shows the strain varies in the circumferential direction and highlights the necessity for 3D models to capture the effects of MPS defects. 


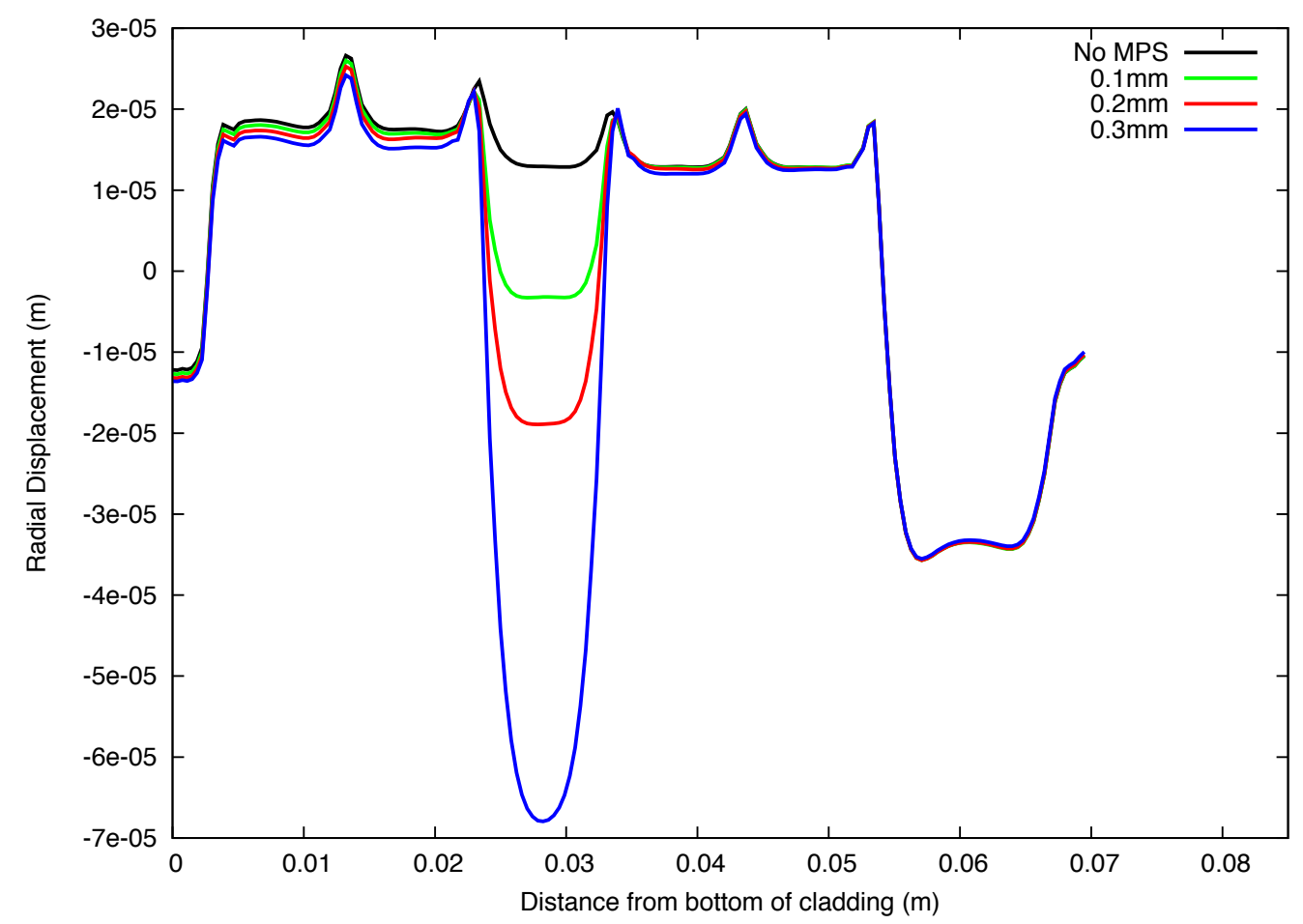

Figure 3.12: Radial cladding deformation with MPS depth variation.

\subsubsection{Conclusions}

BISON simulations were employed to guide the design of a proposed Halden Reactor experiment that will provide useful PCMI validation data for BISON and other fuel performance codes, including unique data of 3D effects associated with MPS pellet defects. These simulations provided information on whether cladding deformations due to the MPS defect will be significant enough to be measurable. In addition, these simulations were used to propose a linear power history that will maximize fuel temperatures, but still maintain the temperature of the in-situ thermocouples below the specified limit. The power history and cladding deformations were determined using full 3D simulations of the fuel rod due to inherent limitations of 2D representations of an MPS defect. A comparison of certain results was made using both frictionless and glued contact models for the pellet-cladding interface in BISON. These comparisons illustrate the effect of the interface contact model, highlight the 3D nature of simulating MPS defects and provide bounding values for the BISON predictions of the experiment. Additional calculations will be performed as more details of the final fuel rod configurations are provided. 




(a)

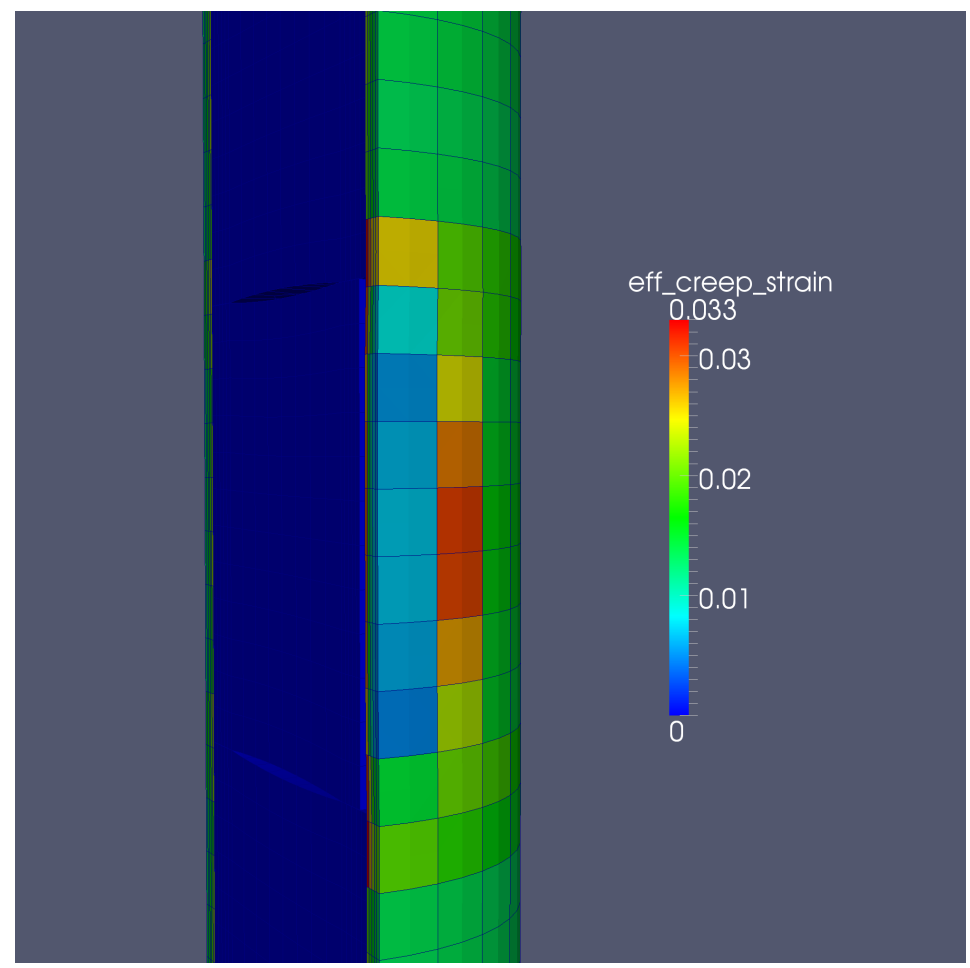

(b)

Figure 3.13: Comparison of cladding effective creep strain computed using frictionless (a) and glued (b) contact. 


\subsection{Pellet Clad Mechanical Interaction Benchmark}

\subsubsection{Background and Introduction}

As described in Section 3.1 and demonstrated by prior international benchmark exercises [15, [16], prediction of cladding diametral strain and elongation is in general a difficult area for fuel performance codes. This has led to the recent initiation of a well-defined PCMI benchmark exercise that is based on four cases, two simplified hypothetical fuel rods and two integral experimental rods from the Halden research reactor. The benchmark includes roughly 20 international participants using 12 different computational tools, either dedicated fuel performance codes or commercial software. The first two cases from the benchmark have been modeled using BISON and predictions were submitted to the benchmark organizers. A coordination meeting was held in Paris in February 2016 where BISON results were compared to other predictions. A brief description of the benchmark cases and some early comparisons are given below.

\subsubsection{Benchmark Cases and Model Status}

Case 1 involves a hypothetical beginning-of-life ramp of a short PWR rodlet (10 pellets) to a rod average power of $40 \mathrm{~kW} / \mathrm{m}$. The ramp occurs over 1 minute (at a constant ramp rate) and is followed by a constant power hold of $100 \mathrm{hrs}$. The ramp-up time is designed to be sufficiently long for thermal transient effects to be negligible, while being sufficiently short for the effects of other time-dependent phenomena to be minimal. A small initial fuel-clad gap is specified such that gap closure occurs part-way up the ramp. BISON predictions were developed assuming both smeared fuel and discrete pellets.

Case 2 is complementary to Case 1, in that it simulates a hypothetical beginning-of-life ramp of a full-length commercial rod to a peak local power of $40 \mathrm{~kW} / \mathrm{m}$. The same power ramp and hold time as for Case 1 are specified. An axial power profile based on a normalized chopped cosine distribution results in a rod average power rating of $27.73 \mathrm{~kW} / \mathrm{m}$ at the end of ramp and during the hold period. As in Case 1, BISON predictions have been developed assuming both smeared and discrete pellets.

Case 3 pertains to experimental rods from the IFA-118 rig which were irradiated in the Halden reactor from 1969 to 1970 . The rods had varying fuel-clad gap sizes, cladding thicknesses, pellet lengths and end-face geometry (flat ended, dished, or chamfered and dished) to enable the effects of these parameters on PCMI to be investigated. Eight sub-cases are included (for eight of the IFA-118 rods) to allow comparison of predictions and measurements for the following:

- in-pile clad elongation as a function of rod average rating during the first ramp to power for otherwise identical rods with different pellet lengths (Sub-cases 3a, 3b and 3c)

- in-pile clad elongation as a function of rod average rating during the first ramp to power for otherwise identical rods with different gap sizes (Sub-cases 3d and 3e)

- in-pile clad elongation as a function of rod average rating during the first ramp to power for otherwise identical rods with different end-face geometries (Sub-cases 3f, 3g and 3h) 
- end-of-life rod elongation, rod diameter and cladding ridge height for otherwise identical rods with different pellet lengths (Sub-cases $3 a, 3 b$ and $3 c$ )

- end-of-life rod elongation, rod diameter and cladding ridge height for otherwise identical rods with different gap sizes (Sub-cases 3d and 3e)

- end-of-life rod elongation, rod diameter and cladding ridge height for otherwise identical rods with different end-face geometries (Sub-cases 3f, $3 \mathrm{~g}$ and $3 \mathrm{~h}$ )

BISON simulations have been prepared for each of the subcases but have not yet been compared to experimental data. Comparisons will be made in early FY17 in preparation for the second benchmark coordination meeting.

Case 4 pertains to IFA-629.4 rod 7, which is a segment of a base-irradiated commercial PWR rod that was subjected to power ramping in the Halden reactor. A simplified base irradiation power history is specified for five cycles. The ramp history has not yet been provided but will correspond to the measured history in the Halden experiment. Model development and comparison to experimental data and other predictions is planned for FY17.

\subsubsection{Early Results and Comparisons}

Early comparisons from this benchmark for Case 1 were recently published in [32]. Figures 3.14 and 3.15, taken from [32], show time histories of the maximum (axially) clad outer diameter and hoop stress, respectively. Comparisons from the twenty different predictions are shown, with the INL BISON prediction given as number 6 . There is obviously a very wide distribution in results from the various participants, attesting to the fact that there remains large uncertainty in PCMI modeling, even for a very simple hypothetical power ramp case. Note that BISON falls near the center of the set of predictions for both the clad diameter and stress.

A single BISON result from Case 2 is given in Figure 3.16, which shows the predicted clad outer diameter along the length of the fuel rod. The result is for a discrete pellet analysis as exhibited by the clad diameter oscillation corresponding to hour-glassing of individual fuel pellets. Early comparisons to other predictions have been made but are not yet available for publication.

\subsubsection{Summary}

The INL is participating in a PCMI benchmark designed to better understand and improve predictions of PCMI. Four comparison cases have been identified and BISON developers have prepared predictions for the first three. Detailed comparisons have been made and published for Case 1. Comparisons for Case 2 are in progress, with Cases 3 and 4 planned for FY17.

Case 1 comparisons from twenty participants show a very wide distribution in results, attesting to the fact that there remains large uncertainty in PCMI modeling, even for a very simple hypothetical power ramp case. Importantly, BISON compares favorably with reputable legacy fuel performance codes such as ENIGMA and TRANSURANUS. The benchmark will provide a valuable opportunity to interact with other fuel modeling experts and improve the PCMI modeling capability in BISON. 


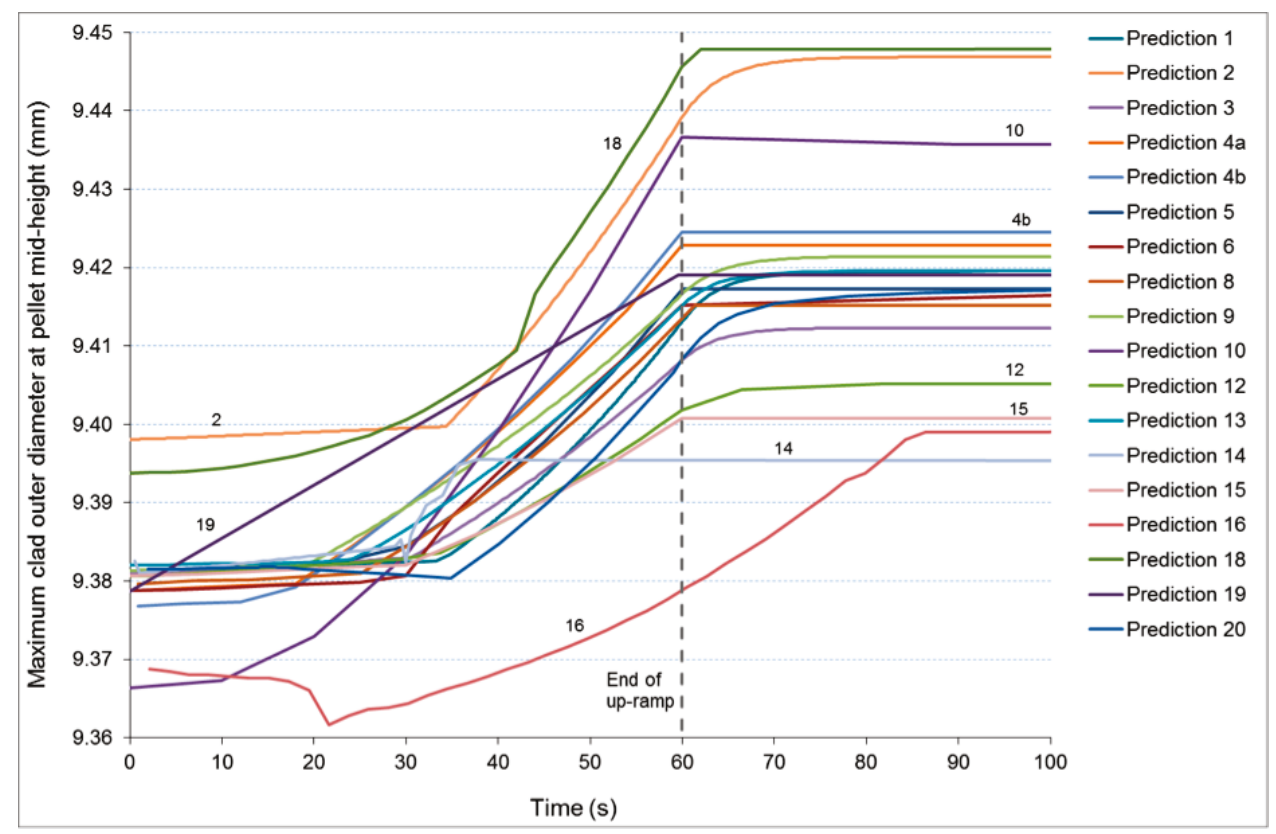

Figure 3.14: Maximum (axially) clad outer diameter versus time predictions for the first $100 \mathrm{~s}$ of Case 1 (where clad outer diameter is evaluated at pellet mid-height).

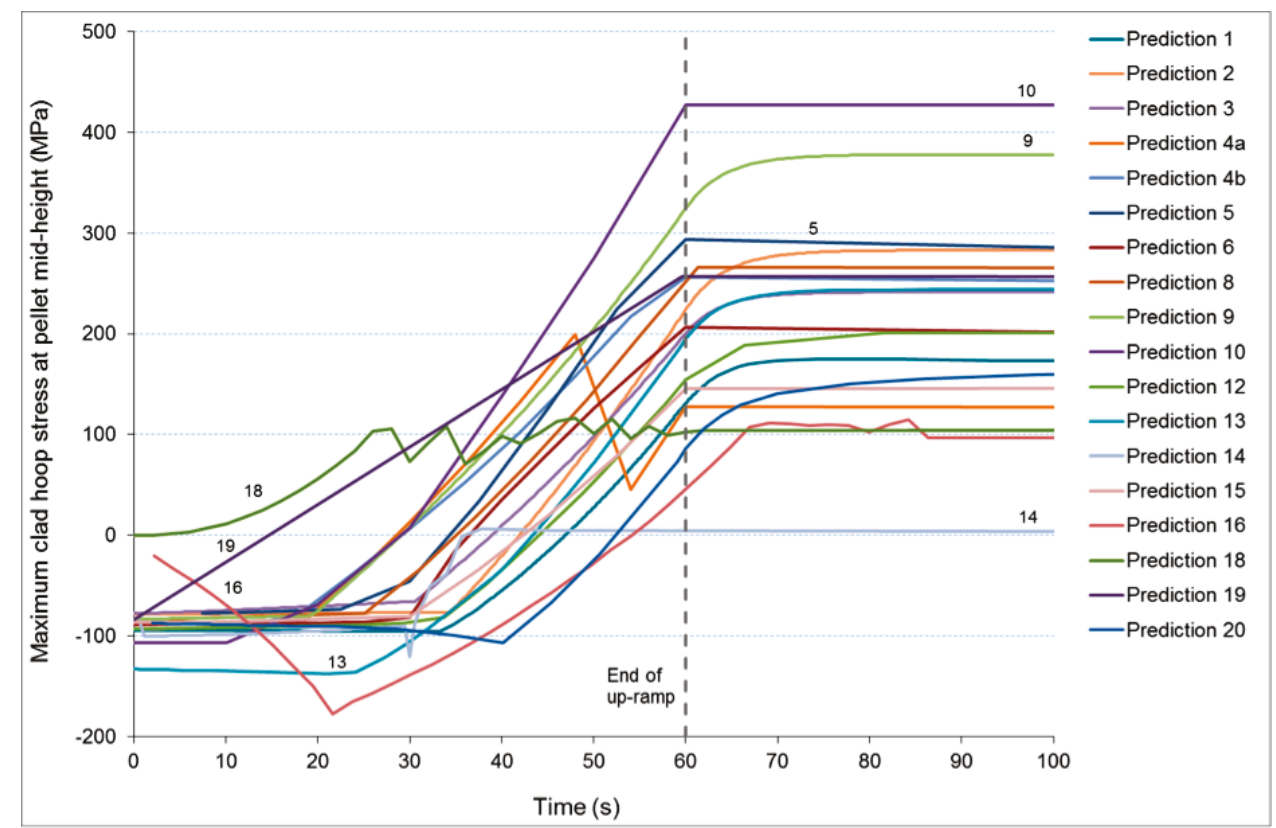

Figure 3.15: Maximum (axially) clad hoop stress versus time predictions for the first $100 \mathrm{~s}$ of Case 1 (where clad hoop stress is evaluated at the clad inner wall and at pellet mid-height). 


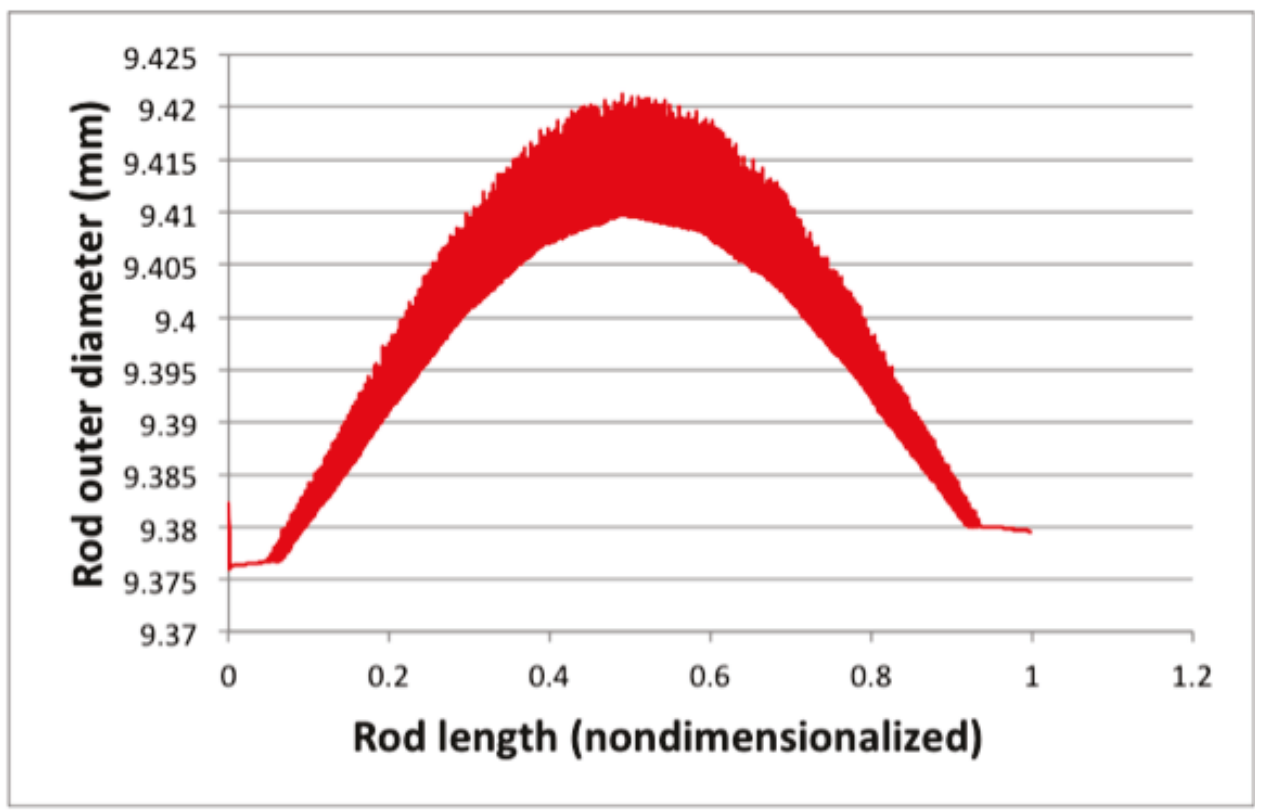

Figure 3.16: BISON predicted clad outer diameter along the length of the fuel rod for Case 2.

\subsection{OECD Reactivity Insertion Accident Benchmark}

\subsubsection{Background and Introduction}

In September 2009 the Organization for Economic Co-operation and Development (OECD)/Nuclear Energy Agency (NEA)/Committee on the Safety of Nuclear Installations (CSNI) organized a technical workshop on Nuclear Fuel Behavior during Reactivity Initiated Accidents. One conclusion from a session in the workshop devoted to RIA safety criteria was that fuel rod performance codes are heavily used during the processes of assessing revised safety criteria for the RIA design basis accident. Therefore, as a conclusion of the workshop it was recommended that a benchmark (RIA benchmark Phase I) between fuel performance codes used for assessing RIAs be organized by the Working Group on Fuel Safety (WGFS).

For the phase I benchmark it was decided to use a set of four experiments on similar highly irradiated fuel rods tested under different conditions. The four experiments were [33]:

- Low temperature, low pressure, stagnant water coolant, very short power pulse (NSRR VA-1)

- High temperature, medium pressure, stagnant water coolant, very short power pulse (NSRR VA-3)

- High temperature, low pressure, flowing sodium coolant, larger power pulse (CABRI CIP0-1)

- High temperature, high pressure, flowing water coolant, medium width power pulse (CABRI CIP3-1) 
The results from the RIA benchmark Phase I [33] showed a large variation in the thermal and mechanical behavior. In cases with water boiling, there was considerable scatter in cladding temperatures and cladding hoop strain calculations varied by a factor of 10 . Therefore, as a conclusion of this benchmark, it was recommended to launch a second phase with the following guidelines [34]:

- The emphasis should be on deeper understanding of the differences in modeling of the different codes; in particular, looking for simpler cases than those used in the first exercise was expected to reveal the main reasons for the observed large scatter in some conditions such as coolant boiling.

- Due to the large scatter between the calculations that was shown in the RIA benchmark Phase I, it appears that an assessment of the uncertainty of the results should be performed for the different codes. This should be based on a well-established and shared methodology. This also entailed performing a sensitivity study of results to input parameters to assess the impact of initial state of the rod on the final outcome of the power pulse.

Following these guidelines a second phase of the RIA benchmark was launched in early 2014. This benchmark has been organized into two activities [34], namely: 1) To compare the results of different simulations on simplified cases in order to provide additional bases for understanding the differences in model-ling of the concerned phenomena and 2) Perform an assessment of the uncertainty of the results, in particular, the impact of the initial states and key models on the results of the transient are investigated.

\subsubsection{Benchmark Specifications}

Detailed benchmark specifications were prepared in order to prevent as much as possible the variability between the applied model among the different institutions and codes. The full detailed specifications can be found in [34], but will be summarized below.

Ten cases were defined with an increasing degree of complexity. The first case is focused mainly on the thermal behavior, the second and third cases are focused on the thermo-mechanical behavior, and the fourth through ninth cases added thermal-hydraulic behavior. In the tenth case the thermal and thermal-mechanical models and properties were imposed as close as possible to those used in FRAPTRAN. It was recommended that each code use the standard options for all models. Failure, fuel relocation and oxidation models must be disabled. In order to limit the variability in initial states and properties of high burnup fuel, the cases are limited to a fresh 17x17 PWR type fuel rod described in Figure 3.17. All cases start from ambient conditions and ramp to normal operating conditions during the first 50 seconds and stabilize at those conditions until $100 \mathrm{~s}$, at which point the transient starts. The simulation is concluded at $200 \mathrm{~s}$.

The fuel is composed of standard $\mathrm{UO}_{2}$ fuel pellets with a diameter of $8.26 \mathrm{~mm}$ and a height of $1 \mathrm{~cm}$. No dishing or chamfer is considered in the model. A total fissile column height of $10 \mathrm{~cm}$ is specified resulting in a 10 pellet stack. The fuel has a theoretical density of $10970 \mathrm{~kg} / \mathrm{m}^{3}$ with $4 \%$ porosity. The cladding is standard Zircaloy- 4 material. The fuel rod is initialized with either a $50 \mu \mathrm{m}$ gap or no gap by modifying the clad inner radius. In nine of the cases the fuel and clad are considered bonded (no slipping occurs) when the fuel is in contact with the cladding. One 


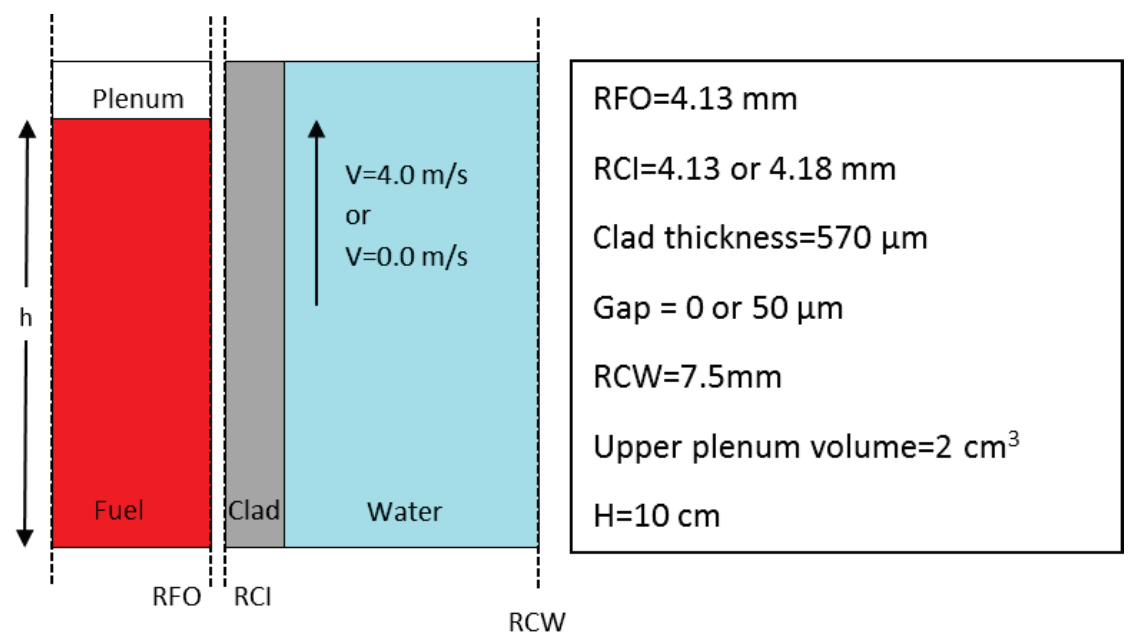

Figure 3.17: Benchmark rod design.

case allows for perfect slipping between the fuel and the cladding when in contact. The plenum volume is defined as $2 \mathrm{~cm}^{3}$ and is filled with helium at either a low value (20 bar) or a high value (50 bar) at $20 \mathrm{C}$.

Depending on the case, the thermal-hydraulic conditions during the transient are:

- PWR Conditions: water coolant at hot zero power (HZP) conditions of $280 \mathrm{C}, 155$ bar and $\mathrm{V}=4.0 \mathrm{~m} / \mathrm{s}$

- BWR Conditions: water coolant at cold zero power (CZP) conditions of $20 \mathrm{C}, 1$ bar and $\mathrm{V}=0.0 \mathrm{~m} / \mathrm{s}$

- Imposed Conditions: during the first 5 seconds of the transient (100-105 s) a bulk coolant temperature of $300 \mathrm{C}$ with an imposed coolant to clad heat transfer coefficient $\mathrm{h}=4,000$ $\mathrm{W} / \mathrm{m}^{2} / \mathrm{K}$. During all remaining times the bulk coolant temperature is $280 \mathrm{C}$ and $\mathrm{h}=40,000$ $\mathrm{W} / \mathrm{m}^{2} / \mathrm{K}$.

- Fixed Conditions: imposed external clad temperature of $280 \mathrm{C}$ and external pressure of $155 \mathrm{bar}$

The power pulse will start from zero power at $\mathrm{t}=100 \mathrm{~s}$ and is approximated with a triangular shape. The pulse width will be $30 \mathrm{~ms}$ full width at half max (FWHM). Two maximum powers will be defined, a low value to avoid departure from nucleate boiling (DNB) and a high value to make DNB possible. For the PWR cases the low value will be $0.4 \mathrm{MW}$ which for the $30 \mathrm{~ms}$ FWHM triangular pulse will result in $50.82 \mathrm{cal} / \mathrm{g}$ of injected energy into the fuel rod. The PWR high value will be $1.0 \mathrm{MW}$ resulting in $127.06 \mathrm{cal} / \mathrm{g}$. For BWR cases the low value will be 0.3 MW (38.12 cal/g) and 1.0 MW (127.06 cal/g). All the power is injected into the $\mathrm{UO}_{2}$ and no contribution will be released in the cladding or coolant. The radial and axial profiles in the fuel are required to be flat. All ten cases are summarized in Figure 3.18. The required parameters to be calculated are outlined in Figure 3.19 . 


\begin{tabular}{|c|c|c|c|c|c|c|c|c|c|c|c|c|c|c|c|}
\hline & & \multicolumn{2}{|c|}{ Geometry } & \multicolumn{2}{|c|}{$\begin{array}{l}\text { Contact } \\
\text { Conditions }\end{array}$} & \multicolumn{2}{|c|}{$\begin{array}{l}\text { Thermomechani } \\
\text { cal Models }\end{array}$} & \multicolumn{4}{|c|}{$\begin{array}{l}\text { Thermal Hydraulic } \\
\text { Conditions }\end{array}$} & \multicolumn{2}{|c|}{$\begin{array}{l}\text { Maximum } \\
\text { Power }\end{array}$} & \multicolumn{2}{|c|}{$\begin{array}{l}\text { Helium } \\
\text { Pressure }\end{array}$} \\
\hline & & No gap & $\begin{array}{c}\text { Open } \\
\text { gap }\end{array}$ & \begin{tabular}{|c|} 
No \\
Slipping
\end{tabular} & slipping & Standard & Imposed & Fixed & PWR & BWR & Imposed & Low & High & Low & High \\
\hline Thermal & case 1 & $\mathrm{x}$ & & $\mathrm{x}$ & & $\mathrm{x}$ & & $\mathrm{x}$ & & & & & $\mathrm{x}$ & $\mathrm{x}$ & \\
\hline \multirow{3}{*}{ Mechanical } & case 2 & & $\mathrm{X}$ & $\mathrm{x}$ & & $\mathrm{x}$ & & $\mathrm{X}$ & & & & & $\mathrm{x}$ & $\mathrm{X}$ & \\
\hline & case 3 & & $\mathrm{x}$ & & $\mathrm{x}$ & $\mathrm{x}$ & & $\mathrm{x}$ & & & & & $\mathrm{x}$ & $\mathrm{x}$ & \\
\hline & case 10 & & $\mathrm{x}$ & $\mathrm{x}$ & & & $\mathrm{x}$ & $\mathrm{x}$ & & & & & $\mathrm{x}$ & $\mathrm{x}$ & \\
\hline \multirow{6}{*}{$\begin{array}{l}\text { Thermal } \\
\text { Hydraulic }\end{array}$} & case 6 & $\mathrm{x}$ & & $\mathrm{x}$ & & $\mathrm{x}$ & & & & $\mathrm{x}$ & & $\mathrm{x}$ & & $\mathrm{x}$ & \\
\hline & case 7 & $\mathrm{x}$ & & $\mathrm{x}$ & & $\mathrm{x}$ & & & & $\mathrm{x}$ & & & $\mathrm{x}$ & $\mathrm{x}$ & \\
\hline & case 4 & $\mathrm{x}$ & & $\mathrm{x}$ & & $\mathrm{x}$ & & & $\mathrm{x}$ & & & $\mathrm{x}$ & & $\mathrm{x}$ & \\
\hline & case 5 & $\mathrm{x}$ & & $\mathrm{x}$ & & $\mathrm{x}$ & & & $\mathrm{x}$ & & & & $\mathrm{x}$ & $\mathrm{x}$ & \\
\hline & case 8 & $\mathrm{x}$ & & $\mathrm{x}$ & & $\mathrm{x}$ & & & $\mathrm{x}$ & & & & $\mathrm{x}$ & & $\mathrm{x}$ \\
\hline & case 9 & $\mathrm{x}$ & & $\mathrm{x}$ & & $\mathrm{x}$ & & & & & $\mathrm{x}$ & & $\mathrm{x}$ & $\mathrm{x}$ & \\
\hline
\end{tabular}

Figure 3.18: Summary of benchmark cases.

\subsubsection{BISON Model}

The geometry described in Figure 3.17 was interpreted into a 2D-RZ model for BISON. Owing to the simplicity of the model specified in the benchmark the internal BISON module (SmearedPelletMesh) was used to generate the mesh. The fuel was defined with 10 radial mesh elements and 40 axial elements. The cladding was defined with 5 radial elements and 40 axial elements. The cladding height was defined to achieve a plenum volume of $2 \mathrm{~cm}^{3}$. The geometry and mesh are shown in Figure 3.20 .

The fuel is assumed elastic with a Young's Modulus of $200 \mathrm{GPa}$, a Poisson ratio of 0.345 and thermal expansion coefficient as defined in MATPRO. The thermal properties of the fuel are defined using the built-in ThermalFuel module with a porosity of 0.04 . The transient power pulse is applied to the fuel as a uniform heat source using the HeatSource module in the BISON Kernels block.

The cladding is modeled using the SolidModel module with the Young's Modulus applied as a function of temperature and a Poisson ratio of 0.3. The thermal properties were defined with the ThermalZry module and the thermal expansion coefficient was applied as a function of temperature from MATPRO. An IsotropicPlasticity module was also applied to the cladding to capture the effect of instantaneous plasticity resulting from the rapid expansion of the fuel into the cladding due to thermal expansion. The yield strength of the cladding was defined as a function of temperature from [5]. No creep models were used due to the small time scales involved in RIA transients.

For cases 4, 5 and 8 that specify PWR Conditions for the thermal-hydraulic conditions the CoolantChannel module in BISON was used. Cases 1,2, and 3 used a fixed temperature on the outside of the cladding. Case 9 required imposed conditions for the bulk coolant temperature and 


\begin{tabular}{|c|c|c|}
\hline Parameter & Unit & Description \\
\hline EDR & $\mathrm{cal} / \mathrm{g}$ & Energy Injected in the whole rodlet as a function of time \\
\hline DHR & $\mathrm{cal} / \mathrm{g}$ & $\begin{array}{l}\text { Variation of radial average enthalpy with respect to initial conditions of the transient } \\
\text { in the rodlet as a function of time (at } \mathrm{z}=\mathrm{h} / 2 \text { ) (please note that: } \mathrm{DHR}(\mathrm{t}=0)=0)\end{array}$ \\
\hline TFC & ${ }^{\circ} \mathrm{C}$ & Temperature of fuel centerline as a function of time $($ at $\mathrm{z}=\mathrm{h} / 2)$ \\
\hline TFO & ${ }^{\circ} \mathrm{C}$ & Temperature of fuel outer surface as a function of time (at $\mathrm{z}=\mathrm{h} / 2$ ) \\
\hline TCI & ${ }^{\circ} \mathrm{C}$ & Temperature of clad inner surface as a function of time (at $z=h / 2)$ \\
\hline $\mathrm{TCO}$ & ${ }^{\circ} \mathrm{C}$ & Temperature of clad outer surface as a function of time (at $\mathrm{z}=\mathrm{h} / 2$ ) \\
\hline $\mathrm{ECMH}$ & $\%$ & $\begin{array}{l}\text { Clad mechanical (elastic }+ \text { plastic) hoop strain at the outer part of the clad as a } \\
\text { function of time (at } \mathrm{z}=\mathrm{h} / 2)\end{array}$ \\
\hline ECMZ & $\%$ & $\begin{array}{l}\text { Clad mechanical (elastic }+ \text { plastic) axial strain at the outer part of the clad as a } \\
\text { function of time (at } \mathrm{z}=\mathrm{h} / 2 \text { ) }\end{array}$ \\
\hline ECTH & $\%$ & $\begin{array}{l}\text { Clad total (thermal }+ \text { elastic }+ \text { plastic) hoop strain at the outer part of the clad as a } \\
\text { function of time }(\text { at } \mathrm{z}=\mathrm{h} / 2)\end{array}$ \\
\hline ECTZ & $\%$ & $\begin{array}{l}\text { Clad total (thermal + elastic }+ \text { plastic) axial strain at the outer part of the clad as a } \\
\text { function of time }(\text { at } \mathrm{z}=\mathrm{h} / 2)\end{array}$ \\
\hline ECT & $\mathrm{mm}$ & Clad total axial elongation as a function of time \\
\hline EFT1 & $\mathrm{mm}$ & Fuel column total axial elongation as a function of time \\
\hline EFT2 & $\mathrm{mm}$ & Fuel column thermal axial elongation as a function of time \\
\hline $\mathrm{SCH}$ & $\mathrm{MPa}$ & Clad hoop stress at outer part of the clad as a function of time (at $\mathrm{z}=\mathrm{h} / 2$ ) \\
\hline $\mathrm{SCZ}$ & $\mathrm{MPa}$ & Clad axial stress at outer part of the clad as a function of time (at $\mathrm{z}=\mathrm{h} / 2$ ) \\
\hline RFO & $\mathrm{mm}$ & Fuel outer radius as a function of time (at $z=h / 2)$ \\
\hline $\mathrm{RCI}$ & $\mathrm{mm}$ & Clad inner radius as a function of time (at $\mathrm{z}=\mathrm{h} / 2$ ) \\
\hline $\mathrm{HFC}$ & $\mathrm{W} / \mathrm{m}^{2} / \mathrm{K}$ & Fuel to clad heat transfer coefficient as a function of time (at $\mathrm{z}=\mathrm{h} / 2$ ) \\
\hline $\mathrm{HCW}$ & $\mathrm{W} / \mathrm{m}^{2} / \mathrm{K}$ & Clad to water heat transfer coefficient as a function of time (at $\mathrm{z}=\mathrm{h} / 2$ ) \\
\hline PG & bar & Free volume pressure as a function of time \\
\hline VOL & $\mathrm{mm}^{3}$ & Free Volume as a function of time (including open porosity) \\
\hline
\end{tabular}

Figure 3.19: Output parameters required for the benchmark.

the convective heat transfer coefficient. This case used the ConvectiveFluxFunction boundary condition with the appropriate temperature and heat transfer coefficient defined above.

Cases 6, 7 and 10 were not completed for this benchmark. Cases 6 and 7 are for BWR CZP conditions which imposes a zero coolant velocity. The BISON CoolantChannel module is not currently applicable for these conditions. Case 10 required modifying all material thermal and mechanical models to match those of FRAPTRAN. Due to time constraints this was not performed. 


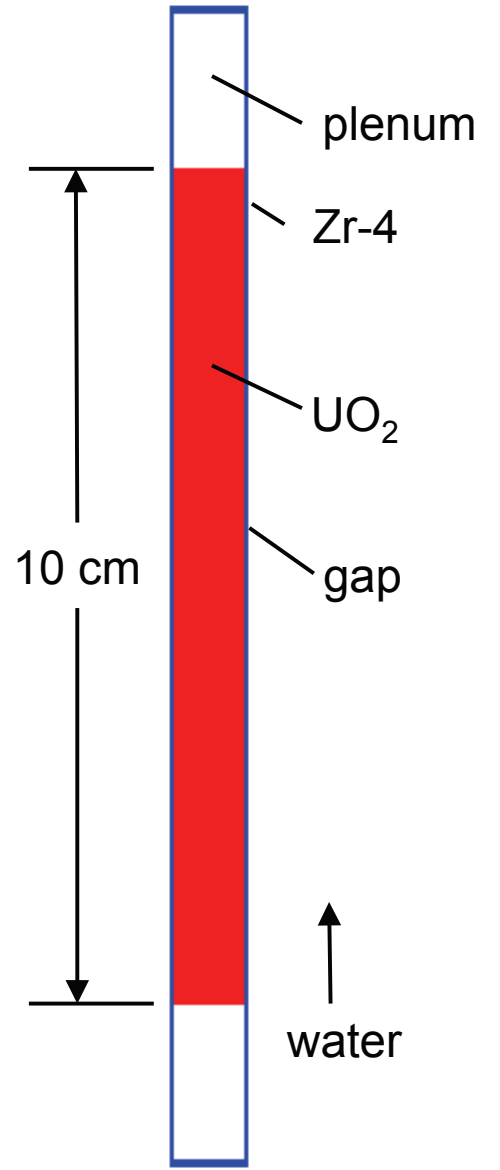

(a)

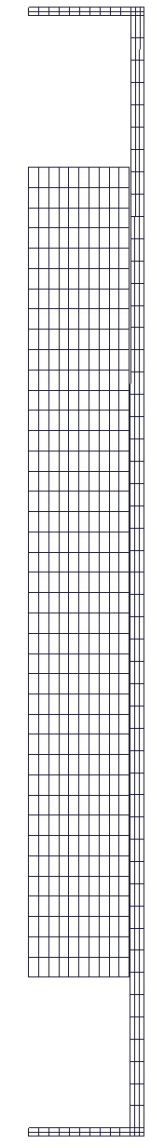

(b)

Figure 3.20: Geometry (a) and 2D axisymmetric mesh (b) for Case 3 of the OECD RIA benchmark exercise. Note that the mesh is magnified $3 \mathrm{x}$ in the radial direction. 


\subsubsection{Results}

A complete compilation of all the results for all cases and codes has been compiled by the OECD [35] comparing each output parameter listed in Figure 3.19. Additionally a more detailed comparison was performed between BISON and FRAPTRAN [36] on prior results before improvements were made to the cladding plasticity model. This report will present and compare the updated results of BISON and FRAPTRAN simulations for case 5 of the benchmark.

An important parameter to consider when discussing RIA transients is the amount of energy injected into the fuel and the resulting fuel radial average enthalpy. Historically the US Nuclear Regulatory Commissions (USNRC) acceptance criterion for reactivity excursions has been based upon the maximum radial average fuel enthalpy in the fuel rod [37]. Therefore, for safety considerations it is necessary to be able to accurately model the fuel radial average enthalpy of the rodlet. The first two parameters of interest in Figure 3.19 were the energy injected into the rodlet and the variation of radial average enthalpy from the starting conditions at time zero. The energy injected, fuel radial average enthalpy, and power pulse are shown in Figure 3.21 . Figure 3.22 shows the fuel radial average enthalpy over a longer duration to show the good agreement between the two codes. Good agreement on the radial average enthalpy of the fuel shows that both codes are calculating comparable radial profiles throughout the fuel pellet during the entire simulation.

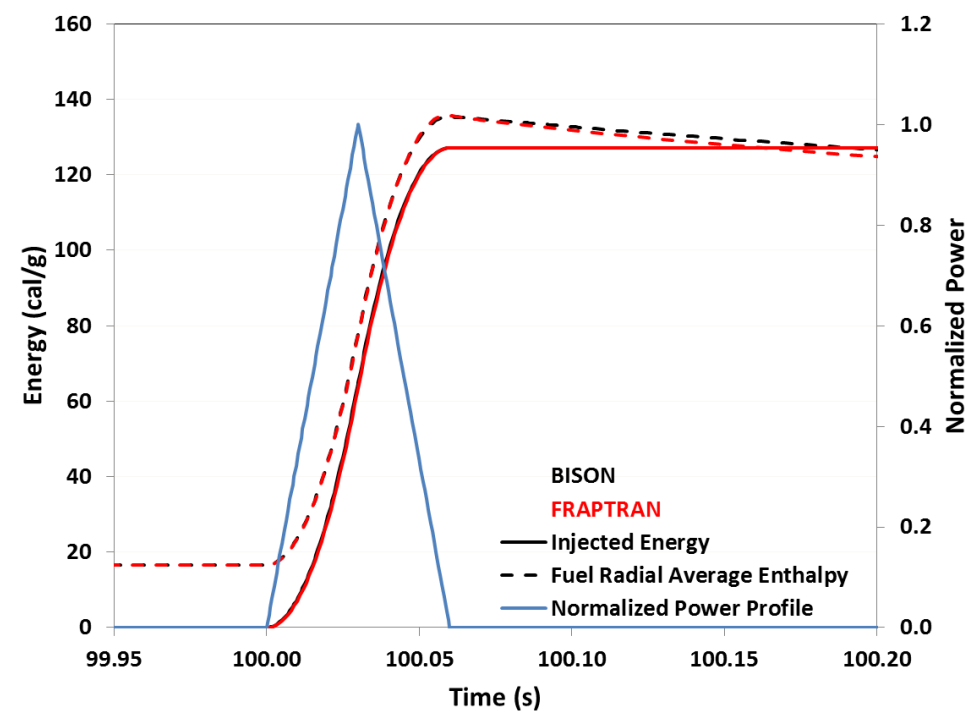

Figure 3.21: Profile comparisons between FRAPTRAN and BISON for the energy injected into the rodlet and fuel radial average enthalpy shown with the simplified 30 ms FWHM power profile.

The temperature profiles at different radial locations in the rodlet are shown in Figure 3.23 The fuel centerline temperature shows good agreement between the two codes over the entire transient. The fuel surface and cladding surface temperatures deviate slightly between the two codes. Due to the complexity of the problem and the multiphysics simulation involved it is 


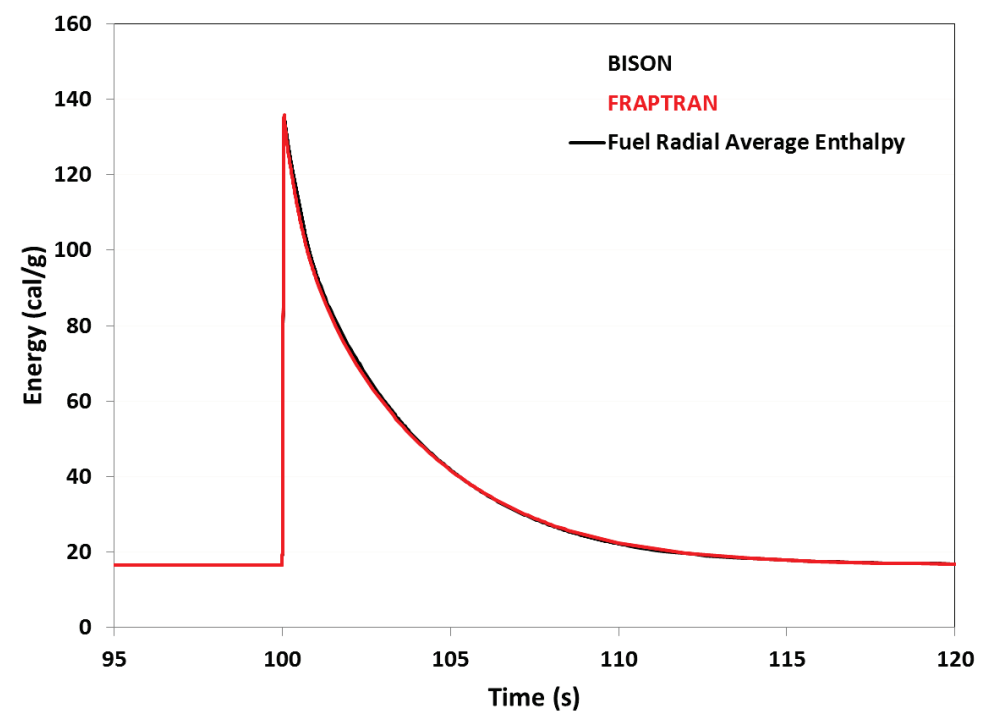

Figure 3.22: Fuel radial average enthalpy shows excellent agreement between BISON and FRAPTRAN.

difficult to pinpoint the cause of the temperature differences between the two codes. There are large variations between the fuel to cladding gap conductance and cladding to coolant heat transfer coefficient between the two codes that will cause differences in temperatures. Also, differences in mechanical models have effects on various mechanisms that affect the energy transport, such as the gap width between the fuel and cladding.
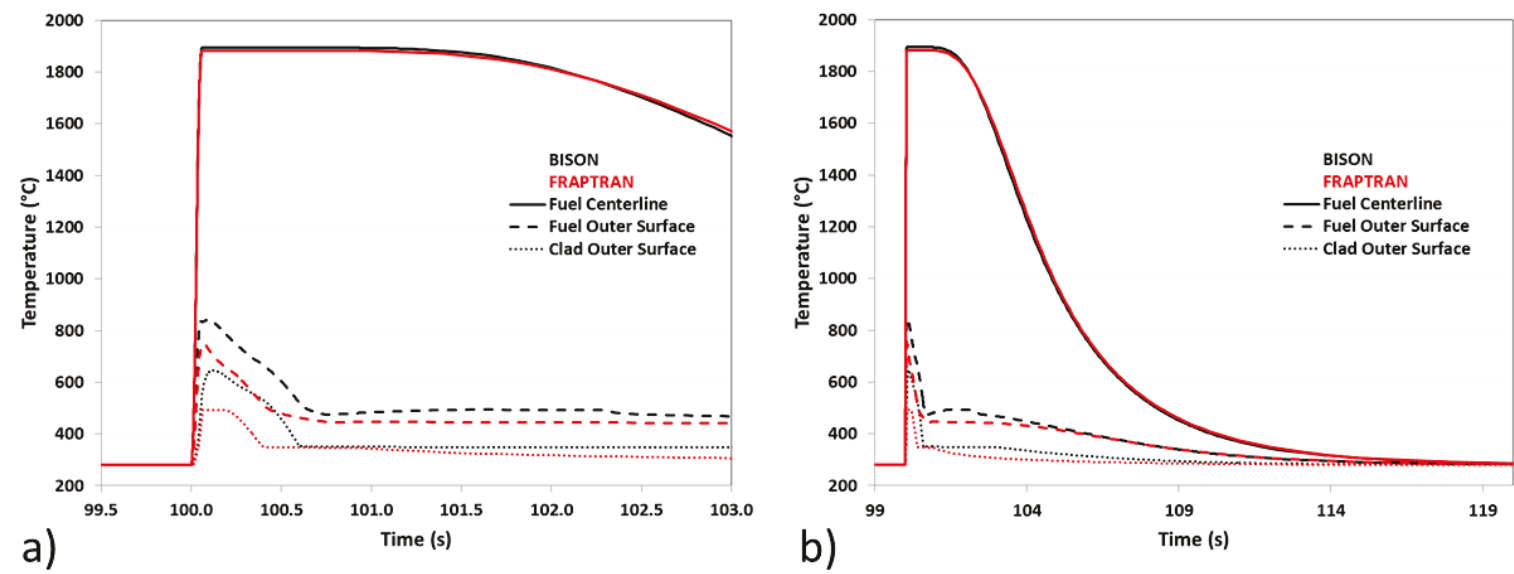

Figure 3.23: Temperature profiles at different radial locations on the rodlet. a) Temperature profiles during a smaller temporal scale around the power pulse and b) larger temporal scale showing temperature profiles during the cooling of the rodlet.

The combination of such a large and rapid temperature increase and the fuel having a co- 
efficient of thermal expansion twice that of the cladding, results in large hoop strains being applied to the cladding. The hoop strain and corresponding hoop stress at the outer surface of the cladding are shown in Figure 3.24. During a RIA event the cladding is forced to expand and conform to the expansion of the fuel, therefore the cladding undergoes a displacement controlled problem. The cladding total hoop strain is controlled by the radial expansion of the fuel until separation occurs during cooling. As such, the total hoop strain shows some variation between the codes. They have very similar evolutions, but FRAPTRAN predicts approximately $0.4 \%$ more strain than BISON after the pulse. This variation correlates to a difference in the maximum outer radius, $4.209 \mathrm{~mm}$ in BISON and $4.219 \mathrm{~mm}$ in FRAPTRAN. This is likely due to multiple reasons. First, FRAPTRAN assumes a rigid pellet that cannot yield, while BISON assumes a compliant fuel pellet. Also differences in fuel thermal expansion and plasticity models between codes could result in the variations in calculated strain.

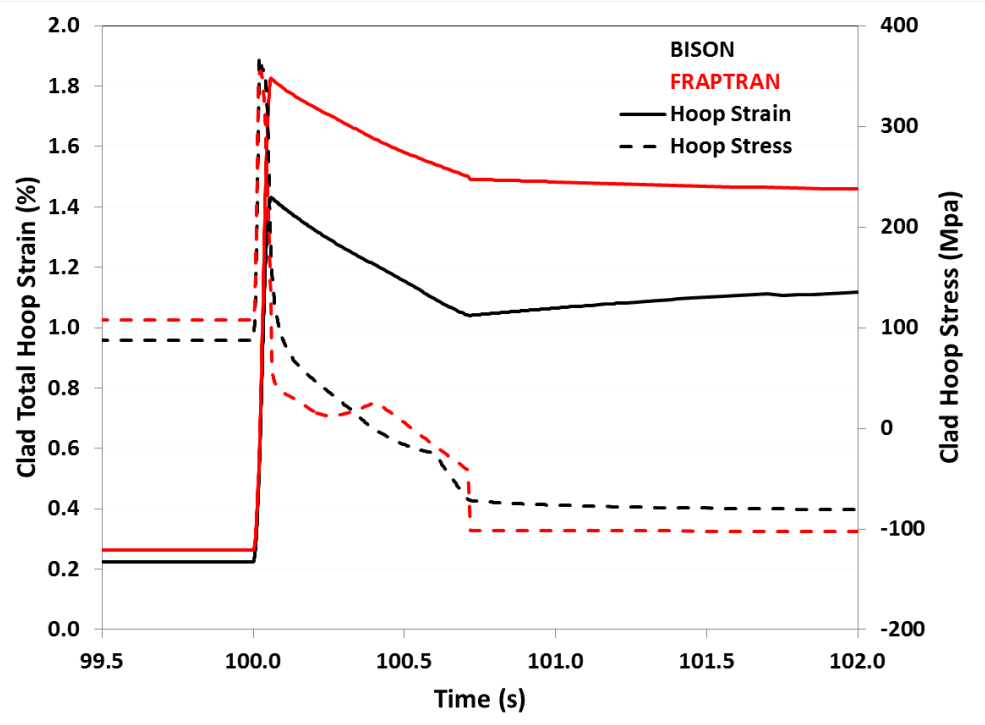

Figure 3.24: Hoop stress and total hoop strain at the outer surface of the cladding.

Each code predicted a maximum hoop stress of approximately $340 \mathrm{MPa}$ and were within $20 \mathrm{MPa}$ of each other throughout the transient. The two codes agree reasonably well on the stress, except for a short time just after the power pulse. During this time just after the pulse (100.06-101.0s) there is a complicated trade-off between elastic strain and the development of plastic strain. The increase in plastic strain is due to the decrease of the Zircaloy yield strength as the temperature increases. The temperature dependent yield strength capability described in Section 2.5 was added to BISON as a result of participation in this OECD benchmark.

\subsubsection{Summary}

The objective of the first task in Phase II of the RIA benchmark was to compare the results of different simulations on ten simplified cases in order to better understand the differences in modeling of the concerned phenomena. INL was able to participate in this international 
collaboration and contribute to seven of the ten cases for the benchmark. The complete results are compiled in an OECD/NEA/CSNI report. The results for case 5 of the benchmark have been compared more rigorously with the results obtained from the USNRC using FRAPTRAN. In general, the results compare reasonably well in both thermal and mechanical aspects, and differences can likely be explained by variations in thermal and mechanical models applied to the materials.

\subsection{Uncertainty Quantification and Sensitivity Analysis in Fuel Modeling}

In the best-estimate fuel performance modeling community there has been an increased demand in providing confidence bounds taking into account, model, simulation, and experimental uncertainty on the best estimate results. In fiscal year 2016, the BISON team participated in Organization for Economic Cooperation and Development (OECD) benchmark program on uncertainty and best estimate modeling described in an OECD report entitled "Benchmark for Uncertainty Analysis in Modeling (UAM) for Design, Operation, and Safety Analysis of LWRs". The BISON effort focused on the cases outlined in Chapter 2 Definition of Exercise II-1: Fuel Modeling. The report outlines the specifications for a variety of numerical and experimental test cases for PWR, BWR, and VVER reactor conditions for both normal operating and transient scenarios. The BISON team's participation this fiscal year was focused on Case 4a from the report which simulated a BWR under normal operating conditions. The case is based upon rod 1 of the IFA-432 experiment irradiated in the Halden reactor in Norway. To perform the uncertainty quantification and sensitivity analyses BISON was coupled to Sandia's National Laboratories' (SNL) Dakota software. This work was completed in collaboration with Laura Swiler from SNL.

By being part of the Halden Reactor Project and having direct access to the experimental data it was found that the irradiation history, axial peaking factors and cladding surface temperatures provided in the OECD benchmark report differed greatly to the actual experimental data. For the comparisons among other participants at the benchmark meeting the data provided in the benchmark report was used. After the OECD benchmark meeting in June, the uncertainty quantification and sensitivity analysis of rod 1 of IFA-432 was extended to use the real power history and perform additional statistical analyses to determine the most influential input parameters. The results and analysis of this study was the subject of a conference paper in TopFuel 2016 entitled "Uncertainty Quantification and Sensitivity Analysis Applications to Fuel Performance Modeling" by Gamble and Swiler, and summarized here.

The IFA-432 experiment was a heavily instrumented fuel assembly irradiated in the Halden boiling water reactor from 1975 to 1984 . The IFA-432 assembly included six instrumented roads, each with centerline temperature instrumentation at both the top and bottom ends of the fuel column. The fuel rods began irradiation with fresh fuel in them. The rod analyzed in this study was rod 1 which had its upper thermocouple fail at 150 days [38]. Therefore, all centerline temperature investigations correspond to the data obtained by the lower thermocouple. The irradiation history, coolant temperature (applied as a Dirichlet boundary condition to the cladding), and lower thermocouple temperature measurements were condensed using the Power Condense software [39]. Condensation of the data is required due to the frequency at which Halden takes 
their measurements. In order to reduce computational costs, all simulations completed in this study were ended after 5E+7 seconds, or 578 days. Each BISON run took approximately 3 hours on 24 processors on the Falcon high performance computing system at Idaho National Laboratory.

The dimensional and operational parameters provided in Hann et al.'s report [38] and compiled by the OECD benchmark [40] have been used to construct a finite element model for use in BISON. Uncertainty bounds have been assigned to many of the boundary conditions (e.g., system power), manufacturing parameters (e.g, pellet diameter), and models used (e.g., fuel thermal conductivity). The uncertain input parameters, and their associated uncertainties (mean and standard deviation) are shown in Table 3.3. The type of distribution for all input uncertainties is normal. Input parameters defined as scaling factors increase or decrease the associated parameter by a percentage.

Table 3.3: Uncertain input parameters with their mean and standard deviations

\begin{tabular}{|c|c|c|}
\hline Uncertain Input & Mean & Standard Deviation \\
\hline Coolant pressure $(\mathrm{Pa})^{1}$ & $3.45 \mathrm{E}+6$ & 11488.5 \\
\hline System power scaling factor ${ }^{1}$ & 1.0 & 0.16667 \\
\hline Cladding thickness $(\mathrm{m})^{2}$ & $4.70 \mathrm{E}-4$ & $6.67 E-7$ \\
\hline Cladding roughness $(\mathrm{m})^{2}$ & $6.40 \mathrm{E}-7$ & $1 \mathrm{E}-7$ \\
\hline Fuel pellet outer diameter $(\mathrm{m})^{2}$ & $5.34 \mathrm{E}-3$ & $3.56 \mathrm{E}-5$ \\
\hline Fuel density $\left(\mathrm{kg} / \mathrm{m}^{3}\right)^{2}$ & 10409.04 & 31.22712 \\
\hline Fuel pellet roughness $(\mathrm{m})^{2}$ & $2.2 \mathrm{E}-6$ & $1.66667 \mathrm{E}-7$ \\
\hline Rod fill pressure $(\mathrm{Pa})^{1}$ & $1.10 \mathrm{E}+5$ & $1.67 \mathrm{E}+3$ \\
\hline Fuel thermal conductivity scale factor ${ }^{3}$ & 1.0 & 0.05 \\
\hline Clad thermal conductivity $(\mathrm{W} / \mathrm{m}-\mathrm{K})^{3}$ & 16.0 & 2.5 \\
\hline Fuel thermal expansion $\left(\mathrm{K}^{-1}\right)^{3}$ & $1.00 \mathrm{E}-5$ & $7.50 \mathrm{E}-7$ \\
\hline Gas conductivity scaling factor ${ }^{3}$ & 1.0 & 0.025 \\
\hline Initial gap thickness $(\mathrm{m})^{2}$ & $1.15 \mathrm{E}-4$ & $9.67 \mathrm{E}-6$ \\
\hline
\end{tabular}

Using the the uncertain input parameters in Table 3.3 a Latin Hypercube Sampling (LHS) study was completed with 200 samples. LHS is a stratified random sampling method where the distribution is divided into strata or bins. Each stratum is chosen to be equally probable, so that the strata are of equal length for uniform distributions but of unequal length for normal distributions. LHS is more efficient than pure Monte Carlo in the sense that it requires fewer samples to achieve the same accuracy in statistics (standard error of the computed mean, for example) [41]. Fourteen outputs were analyzed at 10 particular times throughout the irradiation history. The outputs of interest included the fuel centerline temperature (FCT) at the lower thermocouple, the diameter of the cladding at 10 axial locations, the percent fission gas released (FGR), the average temperature of the cladding, and the gap thickness as it evolves over time. To give the reader a sense of the power profile driving this analysis and also a sense of the 
variability in the centerline temperature results, the sample centerline temperature profiles are plotted in Figure 3.25. During times of peak power, one can see that the FCT can vary by a few hundred degrees, based on the uncertain input parameters defined above. Four of the particular times (in days) that were examined in this study are shown in Figure 3.25. The full set of times examined was: $0.95,6.59,22.99,59.13,132.86,199.20,202.20,290.60,384.42$, and 509.74 days.

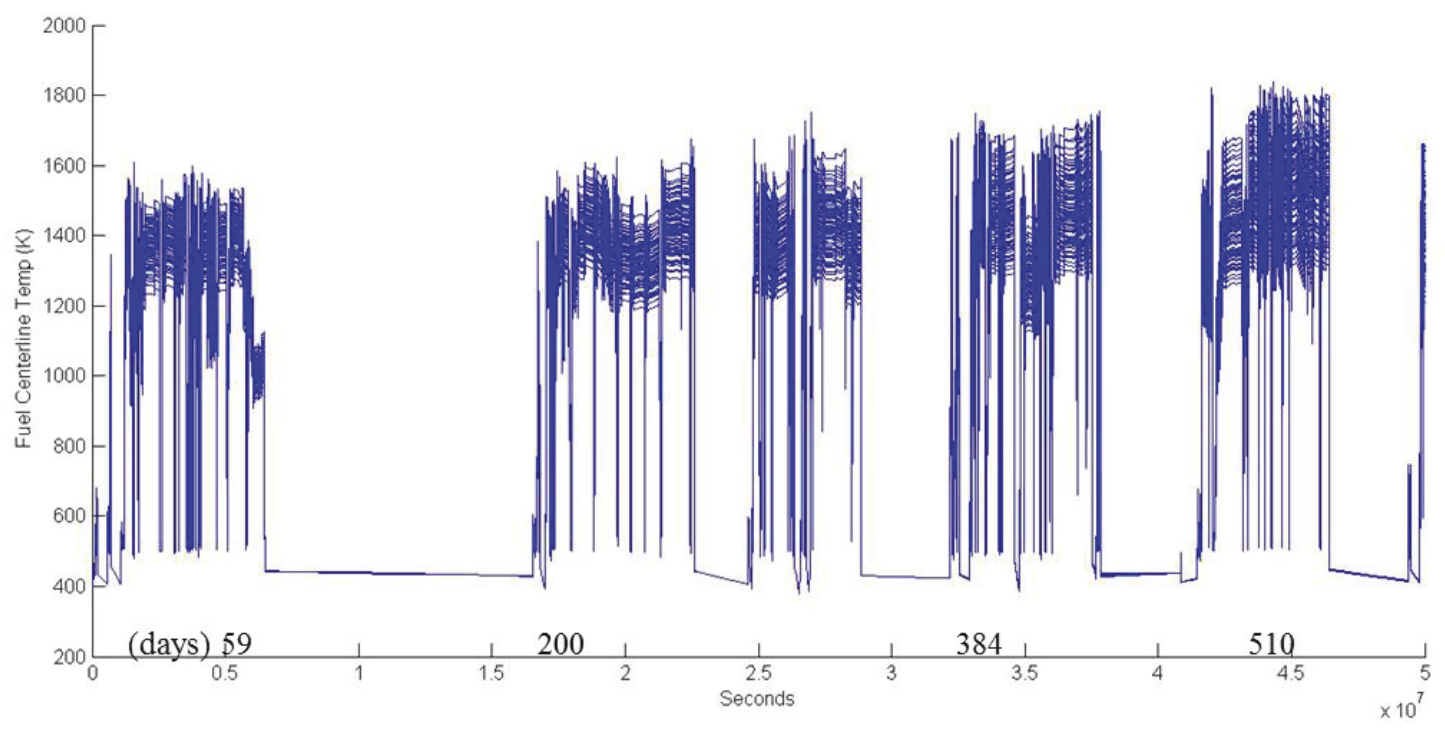

Figure 3.25: Fuel centerline temperature sample results.

For each output, the mean and standard deviation over the 200 samples was calculated. A subset of these are shown in Table 3.4. Certain trends are observed, such that the standard deviation of the FCT increases as a function of irradiation time, especially at high mean FCTs. Moreover, the fission gas released is largest at the end of the experiment with significant uncertainty.

Table 3.4: Selected uncertain outputs with their mean and standard deviations

\begin{tabular}{r|cccc}
\hline Time (Days) & FCT mean (K) & FCT std dev. (K) & FGR mean (\%) & FGR std dev. (\%) \\
\hline 0.9 & 430.6 & 0.3 & 0.00 & 0.00 \\
6.6 & 533.2 & 4.7 & 0.00 & 0.00 \\
23.0 & 1365.0 & 52.0 & 0.00 & 0.00 \\
59.1 & 1072.8 & 32.8 & 0.01 & 0.02 \\
132.9 & 436.5 & 0.3 & 0.03 & 0.04 \\
199.2 & 551.4 & 3.9 & 0.03 & 0.04 \\
202.2 & 1367.5 & 52.7 & 0.04 & 0.04 \\
290.6 & 1404.5 & 70.3 & 0.28 & 0.19 \\
384.4 & 1286.7 & 69.4 & 0.38 & 0.25 \\
509.7 & 1411.6 & 97.6 & 0.71 & 0.43 \\
\hline
\end{tabular}


In addition to mean and standard deviation, correlation coefficients and variance-based indices were calculated. Correlation coefficients measure the strength and direction of a linear relationship between the input variable and output metric. In this analysis Spearman correlation coefficients were calculated which are performed on the ranks of the data. To obtain the ranks of the data the actual data is rearranged such that it is ordered in ascending order. While correlation coefficients only detect linear or monotonic relationships between inputs and outputs, variance-based indices identify the fraction of the variance in an output that can be attributed to an individual variable alone or with interaction effects [42]. Obtaining variance-based indices is computationally expensive and often the only computational feasible approach to calculate the sensitivity indices is to employ surrogate or emulator models in place of the expensive simulation model. In this study, both quadratic regression models and polynomial chaos expansion were used in the calculation of the sensitivity indices. A subset of the main effects indices obtained using quadratic regression and polynomial chaos expansion are summarized in Tables 3.5 and 3.6 at an irradiation time of 384.42 days, respectively. In both tables, the main effects index that is greater than 0.5 are colored yellow, and the main effects indices that are between 0.1 and 0.5 are colored in orange. In these tables the inputs are shown on the rows and the outputs in the columns.

A careful examination of the two tables indicate that both variance-based indices methods yield almost identical results. This observation is not always true and is an indication of the robustness of the surrogates due to the significant differences in their construction. It is found that the variance in the cladding thermal conductivity contributes to the majority of the variance in the cladding diameters. Moreover, the variance in fuel thermal conductivity contributes around $50 \%$ of the FGR and FCT variances. In addition, the initial as fabricated gap thickness contributes to approximately $20 \%$ of the variance in several outputs, such as FCT, FGR, and the current gap thickness.

Table 3.5: Main effects indices between inputs (rows) and outputs (columns) at 384.42 days, based on quad regression.

\begin{tabular}{l|cccccc}
\hline & FCT & CladDiam1 & CladDiam5 & CladDiam10 & FGR & GapThick \\
\hline sys_pressure & $-8.79 \mathrm{E}-5$ & $1.40 \mathrm{E}-2$ & $1.16 \mathrm{E}-2$ & $2.55 \mathrm{E}-3$ & $-2.90 \mathrm{E}-4$ & $-3.69 \mathrm{E}-5$ \\
sys_pow_fac & $4.77 \mathrm{E}-2$ & $2.75 \mathrm{E}-2$ & $2.54 \mathrm{E}-2$ & $1.13 \mathrm{E}-2$ & $2.01 \mathrm{E}-2$ & $3.96 \mathrm{E}-1$ \\
clad_thick & $8.05 \mathrm{E}-5$ & $4.26 \mathrm{E}-3$ & $3.6 \mathrm{E}-3$ & $7.24 \mathrm{E}-4$ & $-1.51 \mathrm{E}-4$ & $8.77 \mathrm{E}-4$ \\
clad_rough & $6.45 \mathrm{E}-5$ & $5.70 \mathrm{E}-4$ & $4.86 \mathrm{E}-4$ & $6.03 \mathrm{E}-4$ & $1.81 \mathrm{E}-4$ & $-2.22 \mathrm{E}-4$ \\
fp_out_rad & $2.77 \mathrm{E}-4$ & $2.03 \mathrm{E}-4$ & $1.89 \mathrm{E}-4$ & $1.13 \mathrm{E}-2$ & $5.32 \mathrm{E}-4$ & $-7.86 \mathrm{E}-4$ \\
fuel_density & $1.50 \mathrm{E}-2$ & $2.06 \mathrm{E}-3$ & $1.67 \mathrm{E}-3$ & $5.84 \mathrm{E}-4$ & $1.50 \mathrm{E}-2$ & $7.49 \mathrm{E}-3$ \\
fuel_rough & $4.61 \mathrm{E}-4$ & $1.03 \mathrm{E}-4$ & $6.48 \mathrm{E}-5$ & $-1.32 \mathrm{E}-4$ & $7.35 \mathrm{E}-4$ & $1.31 \mathrm{E}-3$ \\
fill_pressure & $8.66 \mathrm{E}-6$ & $2.79 \mathrm{E}-3$ & $2.29 \mathrm{E}-3$ & $6.08 \mathrm{E}-4$ & $-4.61 \mathrm{E}-4$ & $5.69 \mathrm{E}-4$ \\
fuel_condff & $5.13 \mathrm{E}-1$ & $8.14 \mathrm{E}-3$ & $5.12 \mathrm{E}-2$ & $8.14 \mathrm{E}-3$ & $5.00 \mathrm{E}-1$ & $2.39 \mathrm{E}-1$ \\
clad_cond & $9.03 \mathrm{E}-3$ & $7.81 \mathrm{E}-1$ & $8.17 \mathrm{E}-1$ & $7.83 \mathrm{E}-1$ & $1.09 \mathrm{E}-2$ & $6.65 \mathrm{E}-3$ \\
fuel_therm_exp & $9.56 \mathrm{E}-2$ & $2.51 \mathrm{E}-3$ & $2.22 \mathrm{E}-3$ & $6.06 \mathrm{E}-4$ & $1.12 \mathrm{E}-1$ & $1.35 \mathrm{E}-1$ \\
gas_cond_f & $1.12 \mathrm{E}-2$ & $9.81 \mathrm{E}-4$ & $7.62 \mathrm{E}-4$ & $-3.51 \mathrm{E}-4$ & $7.07 \mathrm{E}-3$ & $1.35 \mathrm{E}-3$ \\
intial_gap_thick & $2.76 \mathrm{E}-1$ & $8.05 \mathrm{E}-2$ & $6.57 \mathrm{E}-2$ & $1.70 \mathrm{E}-1$ & $2.53 \mathrm{E}-1$ & $2.04 \mathrm{E}-1$ \\
\hline
\end{tabular}


Table 3.6: Main effects indices between inputs (rows) and outputs (columns) at 384.42 days, based on polynomial chaos expansion.

\begin{tabular}{l|cccccc}
\hline & FCT & CladDiam1 & CladDiam5 & CladDiam10 & FGR & GapThick \\
\hline sys_pressure & $1.15 \mathrm{E}-8$ & $1.40 \mathrm{E}-2$ & $1.16 \mathrm{E}-2$ & $2.53 \mathrm{E}-3$ & $1.97 \mathrm{E} 07$ & $2.94 \mathrm{E}-6$ \\
sys_pow_fac & $5.12 \mathrm{E}-2$ & $3.01 \mathrm{E}-2$ & $2.81 \mathrm{E}-2$ & $1.42 \mathrm{E}-2$ & $1.97 \mathrm{E}-2$ & $4.03 \mathrm{E}-1$ \\
clad_thick & $4.93 \mathrm{E}-8$ & $3.54 \mathrm{E}-3$ & $3.04 \mathrm{E}-3$ & $5.88 \mathrm{E}-4$ & 0.00 & $4.24 \mathrm{E}-6$ \\
clad_rough & $1.89 \mathrm{E}-4$ & $1.64 \mathrm{E}-5$ & $2.16 \mathrm{E}-5$ & $7.22 \mathrm{E}-6$ & $5.88 \mathrm{E}-5$ & $9.42 \mathrm{E}-5$ \\
fp_out_rad & $4.44 \mathrm{E}-5$ & $7.20 \mathrm{E}-5$ & $6.20 \mathrm{E}-5$ & $1.29 \mathrm{E}-2$ & $2.53 \mathrm{E}-5$ & $2.95 \mathrm{E}-7$ \\
fuel_density & $1.20 \mathrm{E}-2$ & $1.48 \mathrm{E}-3$ & $1.17 \mathrm{E}-3$ & $2.19 \mathrm{E}-4$ & $1.10 \mathrm{E}-2$ & $5.60 \mathrm{E}-3$ \\
fuel_rough & $6.22 \mathrm{E}-4$ & $1.91 \mathrm{E}-4$ & $1.68 \mathrm{E}-4$ & $1.37 \mathrm{E}-5$ & $6.77 \mathrm{E}-4$ & $4.52 \mathrm{E}-4$ \\
fill_pressure & $1.13 \mathrm{E}-4$ & $2.50 \mathrm{E}-3$ & $2.07 \mathrm{E}-3$ & $3.15 \mathrm{E}-4$ & $4.93 \mathrm{E}-5$ & $5.72 \mathrm{E}-5$ \\
fuel_cond_f & $5.20 \mathrm{E}-1$ & $6.26 \mathrm{E}-2$ & $5.17 \mathrm{E}-2$ & $7.86 \mathrm{E}-3$ & $5.10 \mathrm{E}-1$ & $2.40 \mathrm{E}-1$ \\
clad_cond & $9.86 \mathrm{E}-3$ & $7.92 \mathrm{E}-1$ & $8.25 \mathrm{E}-1$ & $7.91 \mathrm{E}-1$ & $1.15 \mathrm{E}-2$ & $5.84 \mathrm{E}-3$ \\
fuel_therm_exp & $9.89 \mathrm{E}-2$ & $1.55 \mathrm{E}-3$ & $1.38 \mathrm{E}-3$ & $1.40 \mathrm{E}-4$ & $1.18 \mathrm{E}-1$ & $1.38 \mathrm{E}-1$ \\
gas_cond_f & $1.08 \mathrm{E}-2$ & $2.03 \mathrm{E}-3$ & $1.60 \mathrm{E}-3$ & $2.67 \mathrm{E}-4$ & $6.5 \mathrm{E}-3$ & $2.12 \mathrm{E}-3$ \\
intial_gap_thick & $2.82 \mathrm{E}-1$ & $88.17 \mathrm{E}-2$ & $6.73 \mathrm{E}-2$ & $1.68 \mathrm{E}-1$ & $2.53 \mathrm{E}-1$ & $1.93 \mathrm{E}-1$ \\
\hline
\end{tabular}

In conclusion, the uncertainty quantification and sensitivity analysis of rod 1 from IFA-432 highlighted that the standard deviation of the output parameters increased as irradiation time progressed. In addition, the Spearmen rank correlation coefficients indicate that the system power factor, fuel thermal conductivity, fuel thermal expansion, cladding thermal conductivity, and the initial gap thickness input parameters have the strongest correlations with the outputs of interest. The variance-based indices obtained by both quadratic polynomial regression and polynomial chaos expansion concluded that the same five input parameters above contribute the most the variances in the outputs of FCT, FGR, cladding diameter, cladding temperature and evolving gap thickness.

\subsection{Improved Fission Gas Behavior Model}

During FY-2016, significant improvements have been made to BISON's model for the analysis of fission gas behavior. The BISON fission gas model is built with a physically based approach and incorporates the fundamental features of fission gas behavior, among which are intra-granular gas diffusion, gas behavior at grain boundaries and the eventual fission gas release (FGR) from the grain boundaries to the exterior of the fuel pellet. Details of the original BISON model can be found in [24, 43]. During 2016, new features have been added to the model, both to the intra-granular and the grain-boundary modules. These developments have been shown to improve model's performance and predictive accuracy when compared to experimental data. More details are given in the following subsections. 


\subsubsection{New Algorithm for Fission Gas Diffusion with Improved Accuracy}

Fission gas diffusion from within the grains to the grain boundaries (intra-granular diffusion) is the first and basic step of fission gas release. Modeling of this process requires dedicate numerical algorithms that provide solution of the relevant diffusion equation in time-varying conditions. Algorithms available to date include URGAS and FORMAS, which are the most widely used in fuel performance codes worldwide [44-47]. In particular, the original BISON fission gas model used the FORMAS algorithm from [45]. However, these algorithms present deficiencies and can be inaccurate, depending on the operation conditions they are applied to [44, 47]. The improved numerical modeling of intra-granular fission gas diffusion therefore represents one key issue in order to improve current models of fission gas behavior, including BISON's model.

This issue has been tackled in the past years within the BISON team. This research effort has led to the development of a new and improved numerical algorithm for the solution of the intra-granular diffusion problem, called PolyPole-1. This work has been published recently [47]. The PolyPole-1 algorithm has been demonstrated to be vastly superior than prior state-of-the-art algorithms in terms of accuracy, with no significant increase in the associated computational time [47]. These major achievements are summarized in Figs. 3.26 and 3.27. This improved algorithm has been implemented in BISON and is available in the current version of the code.

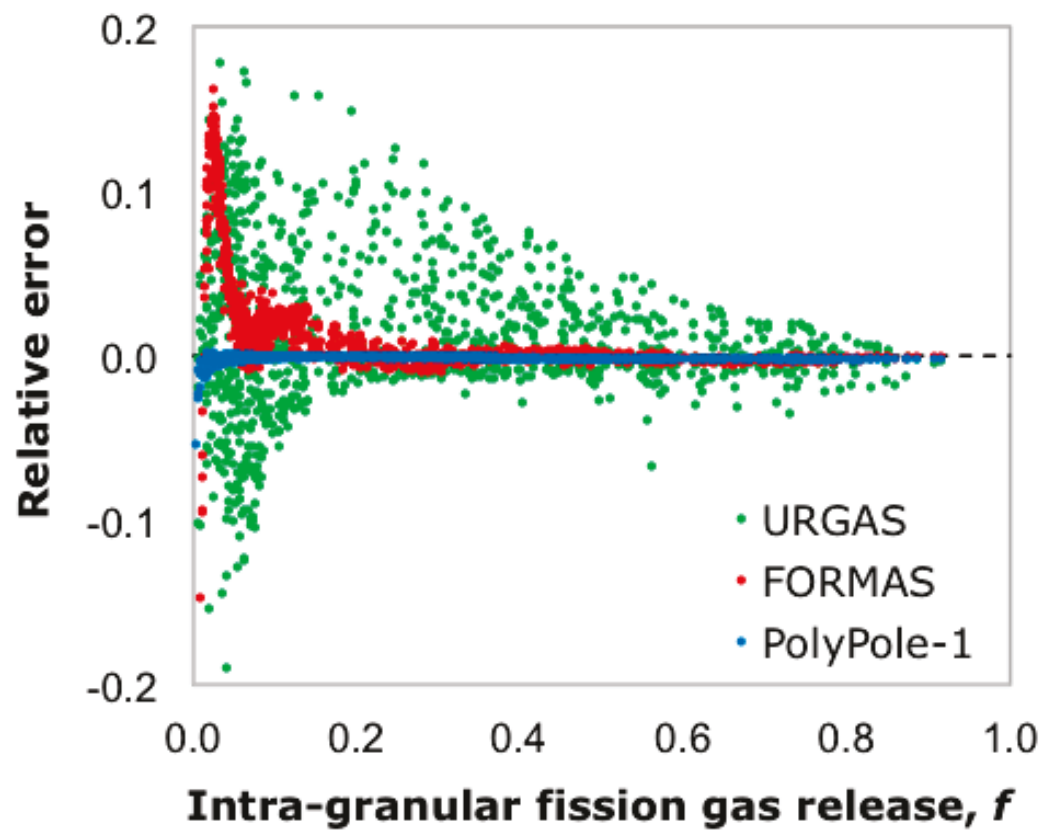

Figure 3.26: Comparison between the fission gas diffusion algorithms URGAS, FORMAS (previous BISON algorithm) and PolyPole-1 (new BISON algorithm) in terms of relative error with respect to the reference solution. Each data point corresponds to one of 1000 calculations with randomly generated conditions.

Details of the PolyPole-1 algorithm's characteristics and verification can be found in [47]. A 


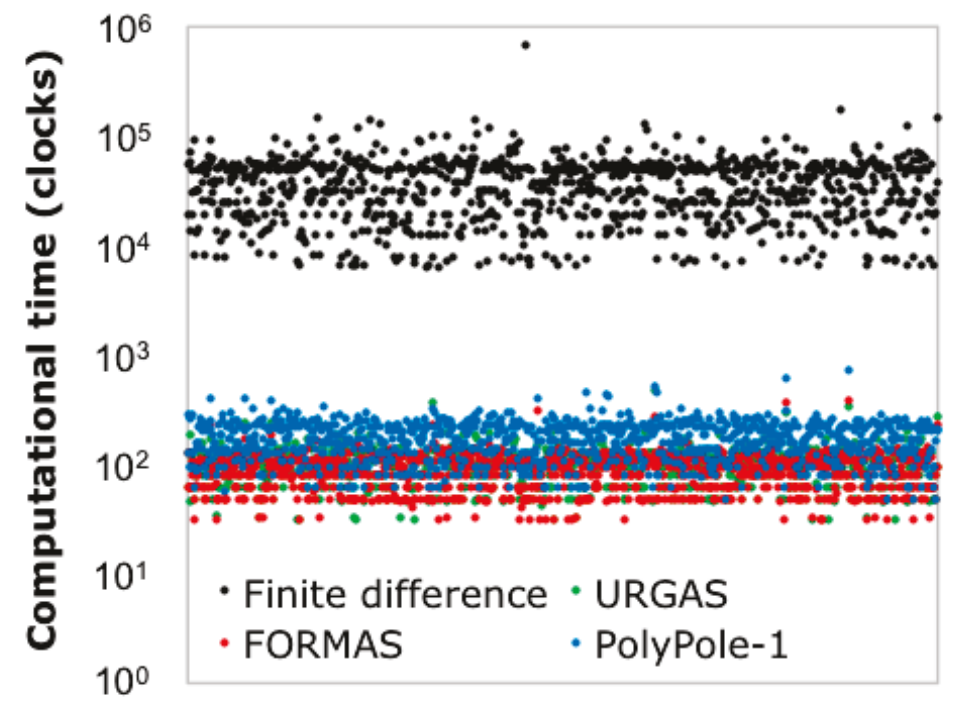

Figure 3.27: Comparison between the computational times associated with the URGAS, FORMAS and PolyPole-1 algorithms, and a finite difference scheme. Each data point corresponds to one of 1000 calculations with randomly generated conditions.

short summary is given hereinafter.

The problem of fission gas diffusion from within the fuel grains (assumed to be spherical) to the grain boundaries can be stated mathematically with a single diffusion equation in onedimensional spherical geometry [48, 49]

$$
\frac{\partial C_{i g}}{\partial t}=\beta+D_{e f f} \nabla^{2} C_{i g}
$$

where $C_{i g}\left(\mathrm{~m}^{-3}\right)$ is the intra-granular gas concentration, $t(\mathrm{~s})$ the time, $\beta\left(\mathrm{m}^{-3} \mathrm{~s}^{-1}\right)$ the gas generation rate, and $D_{\text {eff }}$ is the effective intra-granular diffusion coefficient according to Speight [49]. For the analysis of realistic fission gas behavior problems, solution of Eq. 3.1 in time-varying conditions needs to be computed with dedicated numerical algorithms.

The PolyPole-1 algorithm is based on the analytic modal solution of Eq. 3.1 for constant conditions, with the addition of polynomial corrective terms that embody the information on the deviation from constant conditions. In short, the idea behind the PolyPole-1 approach is that the spatial dependency of the solution for time-varying conditions can be approximated by the spatial dependency of the solution for constant conditions, which is known analytically. Exploiting an analytic representation of the spatial dependency avoids using spatial discretization and is therefore expected to allow for significantly lower computational time compared to spatial discretization methods. In addition, PolyPole-1 adapts the number of modes used to approximate the infinite series in the analytic solution at each time step according to the current conditions. This is a major inherent advantage of PolyPole- 1 compared to other algorithms such 
as FORMAS and URGAS. In particular, it is expected that PolyPole-1 would maintain a more consistent accuracy over different conditions. This has been demonstrated during the verification of the algorithm (Ref. [47] and Fig. 3.26.

The verification of the PolyPole- 1 algorithm is performed through a numerical experiment aimed to (i) assess the accuracy of the PolyPole-1 solution and (ii) compare the accuracy of the PolyPole-1 solution to other state-of-the-art algorithms currently used in fuel performance codes. The numerical experiment consists of application of each algorithm to the numerical solution of Eq. 3.1 for 1000 randomly generated operation histories. Results from the three semi-analytic algorithms are compared to a reference, very high accuracy finite difference (FD) solution [47].

The figure of merit for testing and comparing the algorithms is the fractional intra-granular fission gas release at the end of the considered operation history, defined as

$$
f:=\frac{C_{\text {created }}\left(t_{\text {end }}\right)-C_{\text {ig }}\left(t_{\text {end }}\right)}{C_{\text {created }}\left(t_{\text {end }}\right)}
$$

where $C_{\text {created }}\left(\mathrm{m}^{-3}\right)$ is the concentration of gas created, $C_{i g}\left(\mathrm{~m}^{-3}\right)$ the concentration of intragranular gas (Eq. 3.1), and $t_{\text {end }}$ (s) is the final time of the operation history.

The results of the numerical experiment are presented in Fig. 3.26 for the URGAS, FORMAS, and PolyPole-1 algorithms. Each data point in these figures corresponds to one of 1000 randomly generated operation histories and represents the relative error of the solution (Eq. 3.2) obtained with each algorithm with respect to the FD reference solution. It is evident from the results presented in Fig. 3.26 that the overall accuracy of PolyPole-1 is vastly superior to both FORMAS and URGAS state-of-the-art algorithms. Furthermore, PolyPole-1 overcomes the deficiency at low FGR that characterizes the FORMAS algorithm, which has been used in BISON so far and is the reference algorithm for other well established fuel performance codes (e.g., FALCON [46]). Remarkably, the relative error associated with PolyPole-1 is highly consistent over the whole range of intra-granular fission gas release.

The computational time (i.e., the time took for the analysis of a single operation history) for the three considered algorithms and all histories in the numerical experiment is illustrated in Fig. 3.27. PolyPole-1 requires a computational time similar to other algorithms.

In conclusion, a new fission gas diffusion algorithm for BISON (PolyPole-1) has been developed, verified, and implemented in the code. The results demonstrated that

- The accuracy of the PolyPole-1 solution is high and superior to the algorithm used in BISON so far and to other state-of-the-art algorithms used in fuel performance codes.

- The computational time associated with PolyPole-1 is similar to other algorithms.

- Differently from other algorithms, the accuracy of the PolyPole-1 solution is highly consistent over the whole range of intra-granular fission gas release.

Hence, PolyPole-1 offers a more accurate solution than currently used algorithms, with no significant increase in computational time. The newly developed PolyPole-1 algorithm is available in BISON's fission gas model for the the improved calculation of intra-granular fission gas diffusion, which is the first and basic stage of fission gas release. 


\subsubsection{New Transient Fission Gas Release Capability}

A new capability for transient fission gas release has been recently developed and incorporated in BISON's fission gas model. This model extension allows for FGR during transient tests to be reproduced with significantly higher accuracy compared to BISON's original model. The transient model has been validated against several LWR fuel rod irradiation experiments. Details of model characteristics and validation are presented in [50]. A short summary is given hereinafter.

The final step of fission gas release in nuclear fuel is gas venting from the grain boundaries to the exterior of the fuel pellet. Venting occurs through gas bubble growth and interlinkage at grain boundaries but also through a mechanism of grain-face separation due to micro-cracking, which becomes active during transients (e.g., [51-54]. Micro-cracking is thought to be responsible for the high FGR that is observed in transient tests and is characterized by a rapid kinetics (burst release) [18, 51-53, 55-57]. It follows that, in order to accurately predict fission gas release during transients, capability to account for burst release due to micro-cracking needs to be included in fission gas behavior models.

The new transient FGB capability in BISON is based on the available experimental evidence of the burst release process in oxide fuel. To characterize the micro-cracking mechanism, we developed an empirically based micro-cracking parameter as a suitable temperature-dependent sigmoid function. The model is able to reproduce the common characteristics of burst release observed during the various relevant experimental works reported in the literature, i.e., the process (i) is triggered by temperature variations, (ii) occurs during both heating and cooling transients and (iii) occurs in a limited temperature range. The micro-crack healing process is also accounted for. Model characteristics and equations are discussed in detail in [50].

The model has been validated against 19 LWR fuel rod irradiation experiments from the OECD/NEA International Fuel Performance Experiments (IFPE) database [18]. The power histories of the selected experiments are comprised of a base irradiation under normal operating conditions followed by a ramp test at high power. The considered cases are listed in Table 3.7. Some of these experiments were included in the IAEA Coordinated Research Projects FUMEXII [15] and FUMEX-III [58].

Figure 3.28 shows results of the BISON calculations along with experimental measurements of integral FGR during the AN3 and AN4 ramp tests. Results point out a significant improvement in FGR predictions with the transient capability ( $\mathrm{w} /$ transient model) compared to the original BISON model (w/o transient model). Improvements are both in terms of FGR kinetics and end-of-irradiation FGR values. In particular, differently from the original model, the transient model accounts for the burst release effect, with rapid (burst) release during transients being reproduced.

We also present comparisons of calculations to experimental data of local gas concentrations in the fuel. In particular, we consider profiles of retained xenon concentration across the pellet diameter, measured by X-Ray Fluorescence (XRF) analysis in a fuel sample obtained from the AN3 rod [18]. The comparison between the experimental profile and the BISON calculation, both with the original model and with the transient capability applied, is illustrated in Fig. 3.29 The plot showcases local gas retention in the fuel, which is coupled to gas release, as reproduced by the fission gas behavior model in BISON. In general, a good agreement is observed between the calculated and the measured xenon concentration profiles, with the transient capability tend- 
Table 3.7: Summary of the irradiation experiments from the OECD/NEA IFPE database [18] analyzed with BISON for validation of the new transient FGR capability.

\begin{tabular}{lllll}
\hline Fuel rod & Database & Reactor type & $\begin{array}{l}\text { Average burnup } \\
\left(\mathbf{G W d t}_{U} \mathbf{H}^{-1}\right.\end{array}$ & $\begin{array}{l}\text { FGR, } \\
\text { measured (\%) }\end{array}$ \\
\hline PK1-1 & Super-Ramp & PWR & 35.4 & 8.5 \\
PK1-2 & Super-Ramp & PWR & 35.6 & 13.6 \\
PK1-3 & Super-Ramp & PWR & 35.2 & 22.1 \\
PK1-4 & Super-Ramp & PWR & 33.1 & 13.0 \\
PK2-1 & Super-Ramp & PWR & 45.2 & 28.0 \\
PK2-2 & Super-Ramp & PWR & 45.1 & 32.1 \\
PK2-3 & Super-Ramp & PWR & 44.6 & 44.9 \\
PK2-4 & Super-Ramp & PWR & 41.4 & 9.5 \\
PK6-2 & Super-Ramp & PWR & 36.8 & 3.5 \\
PK6-3 & Super-Ramp & PWR & 36.5 & 6.7 \\
PK6-S & Super-Ramp & PWR & 35.9 & 6.1 \\
\hline AN2 & Ris $\varnothing-3$ & PWR & 43.2 & 29.7 \\
AN3 & Ris $\varnothing-3$ & PWR & 44.0 & 35.5 \\
AN4 & Ris $\emptyset-3$ & PWR & 44.1 & 40.9 \\
AN8 & Ris $\emptyset-3$ & PWR & 43.2 & 13.7 \\
\hline GE7 & Ris $\emptyset-3$ & BWR & 41.7 & 14.4 \\
II3 & Ris $\emptyset-3$ & BWR & 16.3 & 17.4 \\
\hline Rod 8 & IFA 597.3 & BWR & 70 & 15.8 \\
\hline L10 & Regate & PWR & 53.4 & 10.2 \\
\hline
\end{tabular}

ing to improve the results compared to the original model.

Finally, we present simulation results in terms of integral FGR at the end of fuel rod irradiation for all 19 experiments analyzed with BISON for validation of the new transient capability (Table 3.7). The comparisons of BISON calculations to experimental data are shown in Fig. 3.30. Data are tabulated in Table 3.8. Both results obtained with the original BISON fission gas model (i.e., w/o transient model) and those obtained applying the transient capability (w/ transient model) are shown. Comparisons demonstrate that, when the transient model is applied, an improvement of the overall results is obtained. To provide a quantitative estimation of the improvement brought about by the transient model, we computed the relative error of each simulation result with respect to the measured FGR, i.e., $\left(\mathrm{FGR}_{\text {calculated }}-\mathrm{FGR}_{\text {measured }}\right) / \mathrm{FGR}_{\text {measured }}$. The average of the absolute values of this error is a measurement of the overall accuracy. With the original BISON model, the average relative error over all considered cases is of about $50 \%$. Average error reduces to approximately $35 \%$ with the new transient capability applied.

From the validation results presented above, we conclude that the newly developed transient fission gas release capability implemented in BISON's fission gas model significantly improves predictions in terms of (i) kinetics of FGR during transients, (ii) local fission gas concentration retained in the fuel and (iii) integral fuel rod fission gas release at the end of irradiation. 

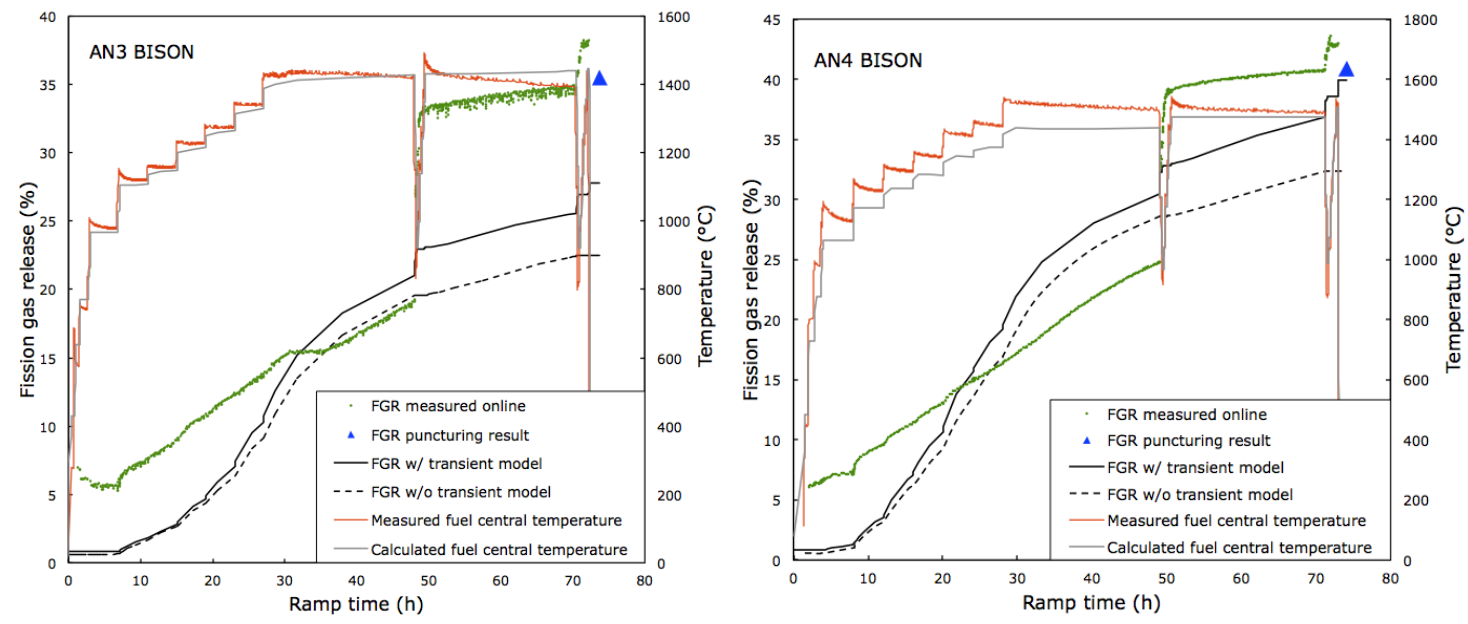

Figure 3.28: Comparison of experimental measurements and BISON calculations of FGR and fuel central temperature as a function of time for the fuel rods AN3 (left) and AN4 (right). FGR results obtained with (w/) and without (w/o) the new transient capability applied are presented.

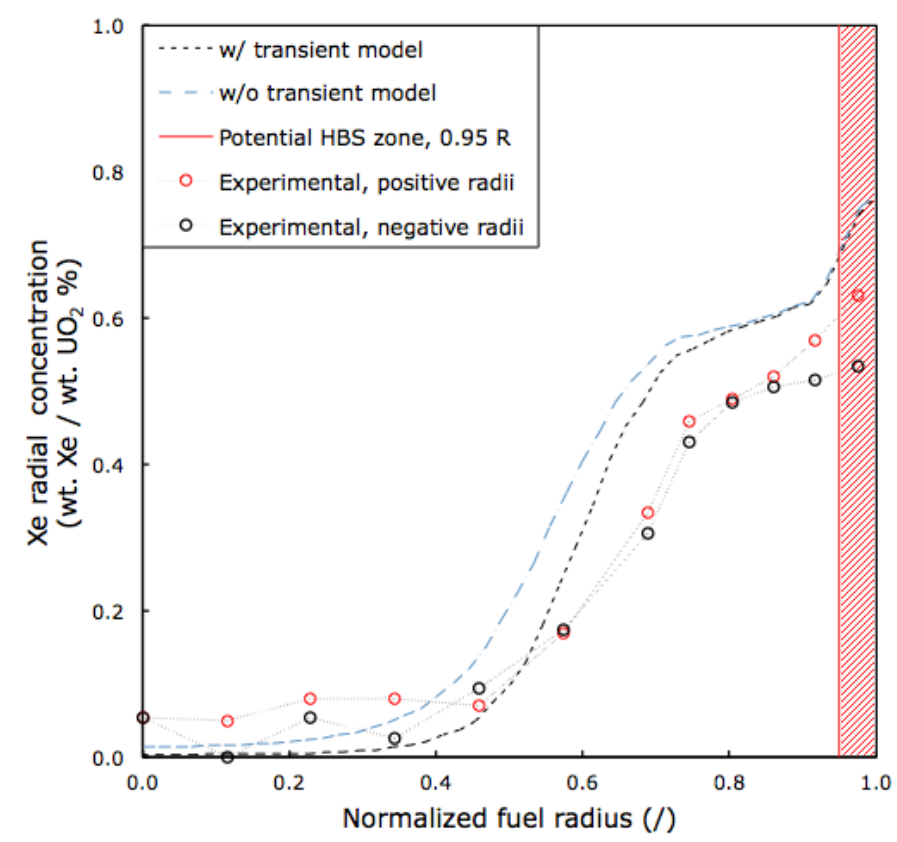

Figure 3.29: Radial profiles of xenon concentration in the AN3 rod. Experimental data are compared to calculations with BISON. Results obtained with (w/) and without (w/o) the new transient capability applied are presented. 
Table 3.8: Summary of integral FGR results obtained with BISON for the 19 LWR fuel rod irradiation experiments analyzed in this work.

\begin{tabular}{llll}
\hline Fuel rod & FGR $(\%)$, measured & $\begin{array}{l}\text { FGR }(\%), \text { calculated } \\
\text { w/o transient model }\end{array}$ & $\begin{array}{l}\text { FGR }(\%), \text { calculated } \\
\text { w/transient model }\end{array}$ \\
\hline PK1-1 & 8.5 & 5.6 & 8.1 \\
PK1-2 & 13.6 & 8.4 & 11.8 \\
PK1-3 & 22.1 & 14.4 & 18.5 \\
PK1-4 & 13.0 & 12.6 & 16.5 \\
PK2-1 & 28.0 & 8.8 & 12.6 \\
PK2-2 & 32.1 & 16.9 & 22.3 \\
PK2-3 & 44.9 & 23.5 & 28.2 \\
PK2-4 & 9.5 & 2.1 & 5.0 \\
PK6-2 & 3.5 & 0.9 & 1.3 \\
PK6-3 & 6.7 & 1.3 & 1.8 \\
PK6-S & 6.1 & 0.9 & 1.3 \\
\hline AN2 & 29.7 & 23.3 & 26.8 \\
AN3 & 35.5 & 22.5 & 27.8 \\
AN4 & 40.9 & 32.4 & 39.9 \\
AN8 & 13.7 & 6.0 & 8.3 \\
\hline GE7 & 14.4 & 8.9 & 12.3 \\
II3 & 17.4 & 8.8 & 12.7 \\
\hline Rod 8 & 15.8 & 3.7 & 8.4 \\
\hline L10 & 10.2 & 3.3 & 6.2 \\
\hline
\end{tabular}




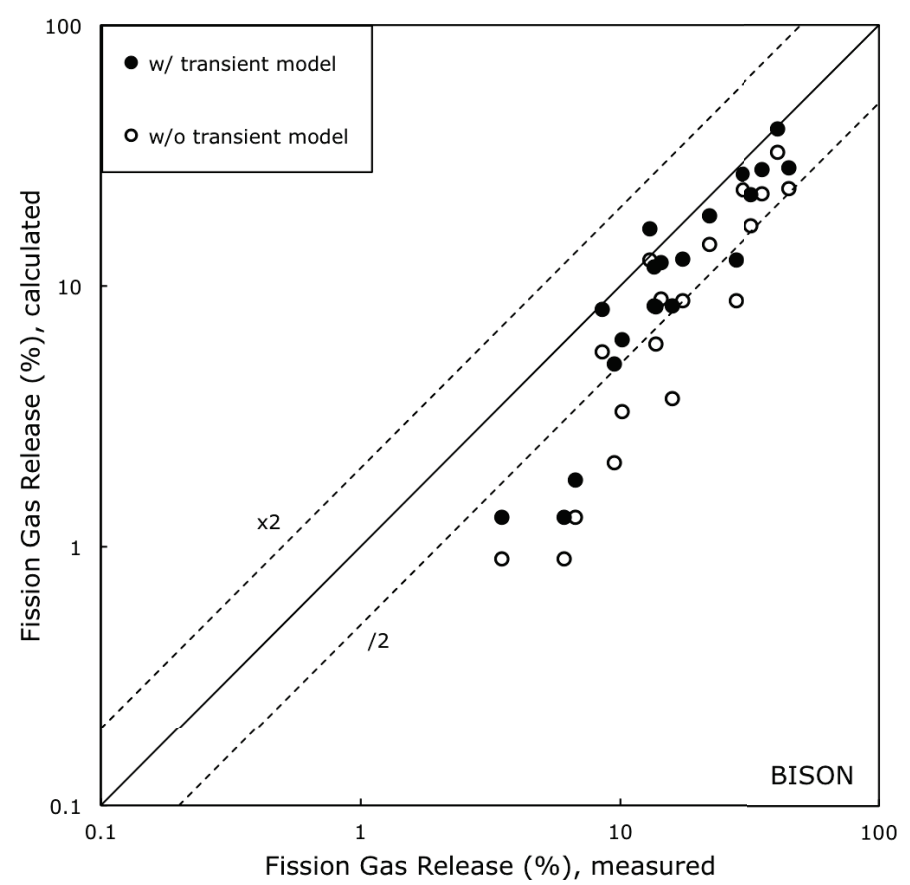

Figure 3.30: BISON results of integral fuel rod FGR compared to experimental data for the 19 LWR fuel rod irradiation experiments analyzed in this work. Results obtained with (w/) and without (w/o) the new transient model applied are presented. 


\section{Future Work}

In FY-2017, the BISON team plans to improve code robustness and efficiency, expand accident simulation capability, continue material model development for fast metal and oxide fuels, improve code documentation and software quality practices, and continue the validation effort.

Key activities include:

- Development of a LOCA demonstration problem to highlight accident capabilities. A Halden LOCA assessment problem (IFA-650.10) will be considered in detail. New capabilities will be developed including steam oxidation of cladding with a moving material interface and anisotropic cladding creep.

- Development of a Used Fuel proof-of-concept, demonstrating BISON's capability to simulate LWR fuel rod behavior from beginning of life through final disposition. Anticipated new capability development will include: 1) hydride formation in Zircaloy cladding (with Marmot support), 2) low temperature creep behavior including hydride effects on creep, 3) cladding failure prediction as a function of hydride concentration and orientation, and 4) convective and radiative heat transfer in a storage environment. Parametric analyses will focus on the drying process during storage.

- LWR validation with a focus on issues related to improving PCMI prediction, specifically cladding creep and fuel swelling. Existing separate effects experiments will be identified and used to update and calibrate key models.

- Conversion of the existing solid mechanics coding to a new and more general tensor mechanics formulation.

- Initial implementation of a new documentation system.

Code cleanup and improvement of software quality practices will remain a priority. The BISON team will provide user support throughout the year via the bison-users@inl.gov group email list, training sessions, and updates to documentation. 


\section{Acknowledgments}

The submitted manuscript has been authored by a contractor of the U.S. Government under Contract DE-AC07-05ID14517. Accordingly, the U.S. Government retains a non-exclusive, royalty free license to publish or reproduce the published form of this contribution, or allow others to do so, for U.S. Government purposes. 


\section{Bibliography}

[1] R. L. Williamson, J. D. Hales, S. R. Novascone, M. R. Tonks, D. R. Gaston, C. J. Permann, D. Andrs, and R. C. Martineau. Multidimensional multiphysics simulation of nuclear fuel behavior. J. Nucl. Mater., 423:149-163, 2012.

[2] J. D. Hales, K. A. Gamble, B. W. Spencer, S. R. Novascone, G. Pastore, W. Liu, D. S. Stafford, R. L. Williamson, D. M. Perez, and R. J. Gardner. BISON users manual. Technical Report INL/MIS-13-30307, Rev. 3, Idaho National Laboratory, September 2015.

[3] J. D. Hales, R. L. Williamson, S. R. Novascone, G. Pastore, B. W. Spencer, D. S. Stafford, K. A. Gamble, D. M. Perez, R.J. Gardner, and W. Liu. BISON theory manual: The equations behind nuclear fuel analysis. Technical Report INL/EXT-13-29930, Rev.2, Idaho National Laboratory, September 2015.

[4] D. M. Perez, R. L. Williamson, S. R. Novascone, R. J. Gardner, K. A. Gamble, A. T. Rice, G. Pastore, J. D. Hales, and B. W. Spencer. Assessment of BISON: A nuclear fuel performance analysis code. Technical Report INL/MIS-13-30314, Rev. 2, Idaho National Laboratory, September 2015.

[5] K. J. Geelhood, C. E. Beyer, and W. G. Luscher. PNNL stress strain correlation for Zircaloy. Technical Report PNNL-17700, Pacific Northwest National Laboratory, 2008.

[6] F. J. Erbacher, H. J. Neitzel, H. Rosinger, H. Schmidt, and K. Wiehr. Burst criterion of Zircaloy fuel claddings in a loss-of-coolant accident. In Zirconium in the Nuclear Industry, Fifth Conference, ASTM STP 754, D.G. Franklin Ed., pages 271-283. American Society for Testing and Materials, 1982.

[7] V. Di Marcello, A. Schubert, J. van de Laar, and P. Van Uffelen. The TRANSURANUS mechanical model for large strain analysis. Nuclear Engineering and Design, 276:19-29, 2014.

[8] E. Perez-Feró, Z. Hózer, T. Novotny, G. Kracz, M. Horváth, I. Nagy, A. Vimi, A. PintérCsordás, Cs. Győri, L. Matus, L. Vasáros, P. Windberg, and L. Maróti. Experimental Database of E110 Claddings under Accident Conditions. Technical Report EK-FRL-2012255-01/02, Centre for Energy Research, Hungarian Academy of Sciences, Budapest, Hungary, May 2013.

[9] A. Bianco, C. Vitanza, M. Seidl, A. Wensauer, W. Faber, and R. Macin-Juan. Experimental investigation on the causes for pellet fragmentation under LOCA conditions. Journal of Nuclear Materials, 465:260 - 267, 2015. 
[10] M. Ek. LOCA testing at Halden, the second experiment IFA-650.2. Technical Report HWR-813, Halden Reactor Project, 2005.

[11] A. Lavoil. LOCA testing at Halden, the tenth experiment IFA-650.10. Technical Report HWR-974, Halden Reactor Project, 2010.

[12] F. J. Erbacher, H. J. Neitzel, and K. Wiehr. Cladding deformation and emergency core cooling of a pressurized water reactor in a LOCA: Summary description of the REBEKA program. Technical Report KfK 4781, Kernforschungszentrum Karlsruhe, Germany, August 1990 .

[13] R.L. Williamson, K.A. Gamble, D.M. Perez, S.R. Novascone, G. Pastore, R.J. Gardner, J.D. Hales, W. Liu, and A. Mai. Validating the BISON fuel performance code to integral LWR experiments. Nuclear Engineering and Design, 301:232 - 244, 2016.

[14] J. D. Hales, S. R. Novascone, B. W. Spencer, R. L. Williamson, G. Pastore, and D. M. Perez. Verification of the BISON fuel performance code. Ann. Nuclear Energy, 71:81-90, 2014.

[15] IAEA. Fuel Modelling at Extened Burnup (FUMEX-II): Report of a Coordinated Research Project 2002-2007. Technical Report IAEA-TECDOC-1687, International Atomic Energy Agency, 2012.

[16] IAEA. Improvement of Computer Codes Used for Fuel Behaviour Simulation (FUMEXIII): Report of a Coordinated Research Project 2008-2012. Technical Report IAEATECDOC-1697, International Atomic Energy Agency, 2013.

[17] C. R. Hann, D. D. Lanning, E. R. Bradley, R. K. Marshall, M. E. Cunningham, and R. E. Williford. Data Report for the NRC/PNL Halden Assembly IFA-432. Technical Report NUREG/CR-0560, PNL-2673, 1978.

[18] E. Sartori, J. Killeen, and J. A. Turnbull. International Fuel Performance Experiments (IFPE) Database. OECD-NEA, 2010, available at http://www.oecdnea.org/science/fuel/ifpelst.html.

[19] T. Tverberg, M. Amaya. Study of thermal behaviour of $\mathrm{UO}_{2}$ and (U, Gd) $\mathrm{O}_{2}$ to high burnup (IFA-515). Technical Report HWR-671, OECD Halden Reactor Project, 2001.

[20] J. D. Hales, D. M. Perez, R. L. Williamson, S. R. Novascone, B. W. Spencer, and R. C. Martineau. Validation of the BISON 3D fuel performance code: Temperature comparisons for concentrically and eccentrically located fuel pellets. In Enlarged Halden Programme Group Meeting: Proceedings of the Fuels and Materials Sessions, volume HPR378, Storefjell Resort Hotel, Norway, March 10-15 2013. OECD Halden Reactor Project.

[21] P. Lösönen. Early-in-life irradiation of IFA-562.2 (the ultra high burn-up experiment). Technical Report HWR-247, OECD Halden Reactor Project, 1989. 
[22] Fuel modelling at extended burnup: Report of the Co-ordinated Research Programme on Fuel Modelling at Extended Burnup - FUMEX 1993-1996. Technical Report IAEATECDOC-998, 1998.

[23] L. C. Bernard, J. L. Jacoud, and P. Vesco. An efficient model for the analysis of fission gas release. Journal of Nuclear Materials, 302:125-134, 2002.

[24] G. Pastore, L. P. Swiler, J. D. Hales, S.R. Novascone, D. M. Perez, B. W. Spencer, L. Luzzi, P. Van Uffelen, and R. L. Williamson. Uncertainty and sensitivity analysis of fission gas behavior in engineering-scale fuel modeling. Journal of Nuclear Materials, 456:398-408, 2015.

[25] K. J. Geelhood and W. G. Luscher. FRAPCON-3.5: A computer code for the calculation of steady-state, thermal-mechanical behavior of oxide fuel rods for high burnup. Technical Report NUREG/CR-7022, PNNL-19418 Vol.1, Rev 1, 2014.

[26] B.W. Spencer, R.L. Williamson, D.S. Stafford, S.R. Novascone, J.D. Hales, and G. Pastore. $3 \mathrm{~d}$ modeling of missing pellet surface defects in BWR fuel. Nuclear Engineering and Design, 307:155 - 171, 2016.

[27] F. Groeschel, G. Bart, R. Montgomery, and S. K. Yagnik. Failure root cause of a PCI suspect liner fuel rod. In IAEA Techincal Meeting on Fuel Failure in Water Reactors: Causes and Mitigation, Bratislave, Slovakia, Jun 17-21 2002.

[28] M. Billaux and H. Moon. Pellet-cladding mechanical interaction in boiling water reactors. In Proceedings of Pellet-Clad Interaction in Water Reactor Fuels, pages 43-52, Aix-enProvence, France, Mar 9-11 2004.

[29] G. Khvostov, W. Lyon, and M.A. Zimmermann. Application of the FALCON code to PCI induced cladding failure and the effects of missing pellet surface. Annals of Nuclear Energy, 62:398-412, 2013.

[30] Y. Aleshin, C. Beard, G. Mangham, D. Mitchell, E. Malek, and M. Young. The effect of pellet and local power variations on PCI margin. In Proceedings of 2010 LWR Fuel Performance, Orlando, FL, USA, Sep 26-29 2010.

[31] J. S. Lee, J. S. Yoo, H. K. Kim, D. Mitchell, and Y. Aleshin. The mechanical behavior of pellet-cladding with the missing chip under PCMI loadings during power ramp. In 2007 International LWR Fuel Performance Meeting - TopFuel, San Francisco, CA, September 30-October 32007.

[32] G. Rossiter et.al. OECD/NEA benchmark on pellet-clad mechanical interaction modelling with fuel performance codes. In OECD/NEA Workshop on Pellet-Cladding Interaction (PCI) in Water-Cooled Reactors, Lucca, Italy, 22-24 June 2016.

[33] RIA fuel codes benchmark. Technical Report NEA/CSNI/R(2013)7, NEA, OCED, 2013.

[34] Reactivity Insertion Accident (RIA) fuel codes benchmark phase-II report - Volume 2: Task No. 1 specifications. Technical Report NEA/CSNI/R(2016)6, NEA, OCED, 2016. 
[35] Reactivity Insertion Accident (RIA) fuel codes benchmark phase-II report - Volume 1: Simplified cases results, Summary and analysis. Technical Report NEA/CSNI/R(2016)6, NEA, OCED, 2016.

[36] C. Folsom, P. Raynaud, A. Zabriskie, R. Williamson, H. Ban, and D. Wachs. Case comparison between FRAPTRAN and BISON for an idealized RIA of a light water reactor. In American Nuclear Society Winter Meeting, Washington DC, 8-12 November 2015.

[37] Assumptions used for evaluating a control rod ejection accident for pressurized water reactors. Technical Report Regulatory Guide 1.77, US Atomic Energy Commission, 1974.

[38] C.R. Hann, D.D. Lanning, E.R. Bradley, R.K. Marshall, M.E. Cunningham, and R.E. Williford. Data report for the NRC/PNL halden assembly IFA-432. Technical Report NUREG/CR-0560, PNL-2673, 1978.

[39] K. Lassmann, A. Schubert, J. van de Laar, and P. Van Uffelen. The 'Fuel Rod Analysis ToolBox': A general program for preparing the input of a fuel rod performance code. Annals of Nuclear Energy, 81:332-335, 2015.

[40] T. Blyth, N. Porter, M. Avramova, k. Ivanov, E. Royer, E. Sartori, O. Cabellos, H. Feroukhi, and E. Ivanov. Benchmark for uncertainty analysis in modelling UAM for design, operation, and safety analysis of LWRSs. Volume II: Specification and support data for the core cases (Phase II). Technical Report NEA/NSC/DOC (2014), Version 2.0, Nuclear Energy Agency/Nuclear Science Committee of the Organization for Economic Cooperation and Development, 2014.

[41] M. Stein. Large sample properties of simulations using latin hypercube sampling. Technometrics, 29(2):143-151, 1987.

[42] A. Saltelli, K. Chan, and E.M. Scott. Sensitivity Analysis. Wiley New York, 2000.

[43] G. Pastore, L. Luzzi, V. Di Marcello, and P. Van Uffelen. Physics-based modelling of fission gas swelling and release in $\mathrm{UO}_{2}$ applied to integral fuel rod analysis. Nuclear Engineering and Design, 256:75-86, 2013.

[44] K. Lassmann and H. Benk. Numerical algorithms for intragranular fission gas release. Journal of Nuclear Materials, 280:127-135, 2000.

[45] P. Hermansonn and A.R. Massih. An effective method for calculation of diffusive flow in spherical grains. Journal of Nuclear Materials, 304:204-211, 2002.

[46] Fuel Analysis and Licensing Code: FALCON MOD01 - Volume 1: Theoretical and Numerical Bases. Technical Report EPRI-1011307, December 2004.

[47] D. Pizzocri, C. Rabiti, L. Luzzi, T. Barani, P. Van Uffelen, and G. Pastore. PolyPole-1: An accurate numeical algorithm for intra-granular fission gas release. Journal of Nuclear Materials, 478:333-342, 2016. 
[48] A.H. Booth. A method of calculating gas diffusion from $\mathrm{UO}_{2}$ fuel and its application to the X-2-f loop test. Technical Report AECL-496, Atomic Energy of Canada Ltd., 1957.

[49] M.V. Speight. A calculation on the migration of fission gas in material exhibiting precipitation and re-solution of gas atoms under irradiation. Nuclear Science and Engineering, 37:180-185, 1969.

[50] T. Barani, E. Bruschi, D. Pizzocri, G. Pastore, P. Van Uffelen, R.L. Williamson, and L. Luzzi. Analysis of transient fission gas behaviour in oxide fuel using BISON and TRANSURANUS. Journal of Nuclear Materials, 2016. Accepted.

[51] M. J. F. Notley and J. R. MacEwan. Stepwise release of fission gas from $\mathrm{UO}_{2}$ fuel. Nuclear Applications, 2:477, 1966.

[52] R. M. Carroll, J. G. Morgan, R. B. Perez, and O. Sisman. Fission density, burnup, and temperature effects on fission-gas release from $\mathrm{UO}_{2}$. Nuclear Science and Engineering, 38:143-155, 1969.

[53] I. J. Hasting, A. D. Smith, P. J. Fehrenbach, and T. J. Carter. Fission gas release from power-ramped $\mathrm{UO}_{2}$ fuel. Journal of Nuclear Materials, 139:531-543, 1986.

[54] C. T. Walker, P. Knappik, and M. Mogensen. Fission gas release from power-ramped $\mathrm{UO}_{2}$ fuel. Journal of Nuclear Materials, 161:10-23, 1988.

[55] K. Une and S. Kashibe. Fission gas release during post irradiation annealing of BWR fuels. Journal of Nuclear Science and Technology, 27:1002-1016, 1990.

[56] J. Nakamura, M. Suzuki, and H. Uetsuka. Re-irradiation tests of LWR spent fuel at JMTR. In Enlarged Halden Programme Group Meeting, Loen, Norway, May 24-29, 1999.

[57] G. Ducros, Y . Pontillon, and P.P. Malgouyres. Synthesis of the VERCORS experimental program: separate-effect experiments on fission product release, in support of the PHEBUS-FP programme. Annals of Nuclear Energy, 61:75-87, 2013.

[58] Improvement of computer codes used for fuel behaviour simulations (FUMEX-III). Technical Report IAEA-TECDOC-1697, International Atomic Energy Agency, 2013. 